Supporting Information

\title{
Total Synthesis of Conulothiazole A
}

Antoine Nitelet, Phidéline Gérard, Jimmy Bouche and Gwilherm Evano*

Laboratoire de Chimie Organique, Service de Chimie et PhysicoChimie Organiques, Université

libre de Bruxelles (ULB), Avenue F. D. Roosevelt 50, CP160/06, 1050 Brussels, Belgium

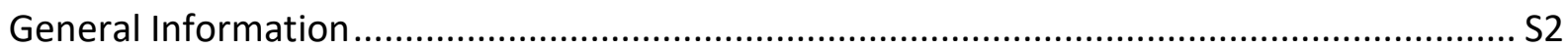

Experimental Procedures and Characterization Data:

Unreported Starting Materials for the Enantioselective Hydrogenation .............................. S3

Experimental Procedures and Characterization Data:

Enantioselective Hydrogenation of 2-Enamido-Heteroarenes ...................................................S8

Experimental Procedures and Characterization Data:

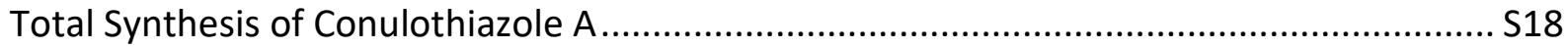

Conulothiazole A Spectral Data Compared to Reported Data ........................................ S27

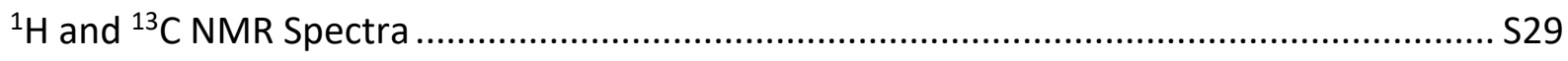




\section{General Information.}

All reactions were carried out in oven-dried glassware under an argon atmosphere employing standard techniques in handling air-sensitive materials.

All solvents were reagent grade or HPLC grade. Tetrahydrofuran was freshly distilled from sodium/benzophenone under argon, dichloromethane and DMF were distilled from calcium hydride under argon. Diethyl ether and toluene were bought over molecular sieves in AcroSeal ${ }^{\circledR}$ bottles from Acros Organics and used as supplied. $n$-Hexane and isopropanol were HPLC grade. Copper(I) iodide (99,999\% purity) and copper(I) bromide (99,999\% purity) were purchased from Aldrich and used as supplied. All other reagents were used as supplied.

Reactions were magnetically stirred and monitored by thin layer chromatography using Merck-Kieselgel $60 \mathrm{~F}_{254}$ plates. Flash chromatography and filtrations were performed with silica gel 60 (particle size $40-63 \mu \mathrm{m}$ ) supplied by Merck. Yields refer to chromatographically and spectroscopically pure compounds, unless otherwise stated.

Proton NMR spectra were recorded using an internal deuterium lock at ambient temperature on Brucker 300 and $600 \mathrm{MHz}$, and on Varian and Jeol $400 \mathrm{MHz}$ spectrometers. Internal reference of $\delta_{\mathrm{H}} 7.26$ was used for $\mathrm{CDCl}_{3}$ and of $\delta_{\mathrm{H}} 3.31$ was used for $\mathrm{CD}_{3} \mathrm{OD}$. Data are presented as follows: chemical shifts (in ppm on the $\delta$ scale relative to $\delta_{\text {TMS }}=0$ ), multiplicity ( $\mathrm{s}$ = singlet, $\mathrm{d}=$ doublet, $\mathrm{t}=$ triplet, $\mathrm{q}=$ quartet, quint. = quintuplet, $\mathrm{m}=$ multiplet, $\mathrm{br} .=$ broad, app. = apparent), coupling constants $(\mathrm{J} / \mathrm{Hz})$ and integration. Resonances that are either partially or fully obscured are denoted obscured (obs.). Carbon-13 NMR spectra were recorded at 75, 100 and $150 \mathrm{MHz}$ using $\mathrm{CDCl}_{3}\left(\delta_{\mathrm{C}} 77.16\right)$ and $\mathrm{CD}_{3} \mathrm{OD}\left(\delta_{\mathrm{C}} 49.00\right)$ as internal references. Fluorine-19 NMR spectra were recorded at $377 \mathrm{MHz}$ using trifluoroethanol- $d_{3}\left(\delta_{F}-77.59\right)$ as an external reference.

Optical rotations were recorded on an Anton Paar Polarimeter MCP100 at $589 \mathrm{~nm}$ and reported as follows: $[\alpha]_{\mathrm{D}}^{20}$, concentration $(c$ in $\mathrm{g} / 100 \mathrm{~mL}$ ), and solvent. Melting points were recorded on a Stuart Scientific Analogue melting point SMP11. Infrared spectra were recorded on a Bruker Alpha spectrophotometer in attenuated total reflection method (ATR). High-resolution mass spectra were recorded on a QTOF Micro WATERS or a Thermo Finnigan MAT 95XP spectrometers. 


\section{Experimental Procedures and Characterization Data:}

\section{Unreported Starting Materials for the Enantioselective Hydrogenation}

\section{General scheme for the synthesis of 2-enamido-heteroarenes:}

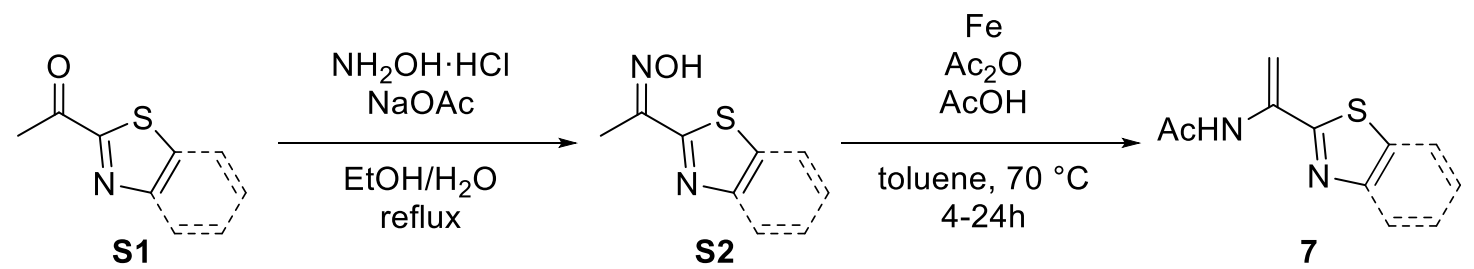

\section{General procedure for the synthesis of unreported ketoximes S2: ${ }^{\mathrm{S}}$}

A round-bottomed flask equipped with a reflux condenser was charged with the ketone S1 (1 equiv.), hydroxylamine hydrochloride (1.5 equiv.) and sodium acetate ( 2.5 equiv.). A mixture of $\mathrm{EtOH} / \mathrm{H}_{2} \mathrm{O}(75 / 25 \mathrm{v} / \mathrm{v}, 0.3 \mathrm{M})$ was next added and the resulting reaction mixture was heated to reflux overnight. The crude reaction mixture was then cooled to room temperature and was poured onto $50 \mathrm{~mL}$ of ice-cooled water. After 30 minutes, the resulting precipitate was filtered, thoroughly washed with water and dried under vacuum to give the pure ketoxime that was used in the next step without further purification.

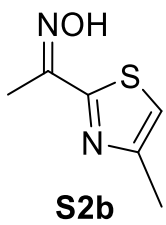

2-[1-(Hydroxyimino)ethyl]-4-methyl-thiazole S2b. Prepared from 2-acetyl-4-methylthiazole S1b (1.0 g, $7.1 \mathrm{mmol}$ ); Yield: 88\% (976 mg, $6.25 \mathrm{mmol}) ; \mathrm{E} / \mathrm{Z}$ ratio: 87/13; White solid; Mp: $130{ }^{\circ} \mathrm{C}$; ${ }^{1} \mathrm{H}$ NMR (400 MHz, $\mathrm{CDCl}_{3}$ ): $\delta 11.25$ (br. s, $\left.0.13 \mathrm{H}\right), 9.02$ (br. s, $\left.0.87 \mathrm{H}\right), 7.14$ (d, $J=0.6 \mathrm{~Hz}, 0.13 \mathrm{H}$ ), $6.85(\mathrm{~d}, J=0.7 \mathrm{~Hz}, 0.87 \mathrm{H}), 2.54(\mathrm{~d}, J=0.7 \mathrm{~Hz}, 0.39 \mathrm{H}), 2.48(\mathrm{~d}, J=0.8 \mathrm{~Hz}, 2.61 \mathrm{H}), 2.47(\mathrm{~s}, 0.39 \mathrm{H})$, $2.42(\mathrm{~s}, 2.61 \mathrm{H}) ;{ }^{13} \mathrm{C} \mathrm{NMR}\left(100 \mathrm{MHz}, \mathrm{CDCl}_{3}\right): \delta 164.7(E), 156.3(Z), 153.4(E), 153.2(E), 152.5(Z)$,

\footnotetext{
S1 Procedure adapted from: Phatake, R. S.; Patel, P.; Ramana, C. V. Org. Lett. 2016, 18, 292.
} 
$146.8(Z), 117.9(Z), 114.8(E), 19.2(Z), 17.2(E), 17.1(Z), 11.6(E)$; IR (ATR) $v_{\max }$ 2856, 1537, 1435, 1026, 907, 753, $668 \mathrm{~cm}^{-1}$; ESIHRMS m/z calcd for $\mathrm{C}_{6} \mathrm{H}_{9} \mathrm{~N}_{2} \mathrm{OS}[\mathrm{M}+\mathrm{H}]^{+} 157.0430$, found 157.0426 .

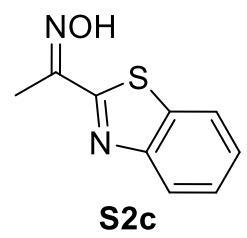

2-[1-(Hydroxyimino)ethyl]-benzo[d]thiazole S2c. Prepared from 2-acetyl-benzo[d]thiazole S1c (1.0 g, $5.6 \mathrm{mmol}) ;$ Yield: 99\% (1.1 g, $5.6 \mathrm{mmol}) ; \mathrm{E} / \mathrm{Z}$ ratio: $93 / 7$. White solid; Mp: $180{ }^{\circ} \mathrm{C} ;{ }^{1} \mathrm{H} \mathrm{NMR}$ (400 MHz, DMSO-d $)_{6}: \delta 12.65$ (s, 0.7H), 12.25 (s, 0.93H), 8.18-8.11 (m, 0.15H), 8.09-8.00 (m, 1.85H), 7.62-7.42 (m, 2H), 2.46 (s, 0.21H), $2.33(\mathrm{~s}, 2.79 \mathrm{H}) ;{ }^{13} \mathrm{C}$ NMR (100 MHz, DMSO-d 6 ): $\delta 166.1$ $(E), 155.3(Z), 152.7(E), 151.4(E), 150.3(Z), 146.9(Z), 135.6(Z), 134.0(E), 126.6(Z), 126.3(E)$, $126.3(Z), 126.2(E), 123.5(Z), 123.0(E), 122.1(E), 18.8(Z), 11.0(E)$; IR (ATR) $v_{\max }$ 2865, 1502, $1457,1324,1026,1013,920,758,730,716 \mathrm{~cm}^{-1}$; ESIHRMS $\mathrm{m} / \mathrm{z}$ calcd for $\mathrm{C}_{9} \mathrm{H}_{9} \mathrm{~N}_{2} \mathrm{OS}[\mathrm{M}+\mathrm{H}]^{+}$ 193.0430, found 193.0427. 


\section{General procedure for the synthesis of 2-enamido-heteroarenes 7: ${ }^{22}$}

Acetic anhydride ( 2 equiv.) and acetic acid ( 3 equiv.) were added to a solution of the ketoxime (1 equiv.) in THF (0.2 M). The solution was purged with argon for 15 minutes. Iron (2 equiv.) was next added and the resulting reaction mixture was stirred at $70{ }^{\circ} \mathrm{C}$ for $4-24$ hours under argon. The crude reaction mixture was cooled to room temperature, quenched with a saturated aqueous solution of $\mathrm{NaHCO}_{3}$ and extracted thrice with ethyl acetate. The combined organic layers were successively washed with a saturated aqueous solution of $\mathrm{NaHCO}_{3}$ and brine, dried over anhydrous magnesium sulfate, filtered and concentrated. The crude residue was finally purified by flash column chromatography over silica gel.

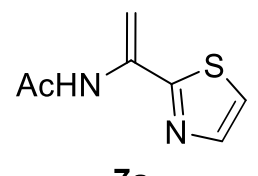

$7 a$

2-(Acetamido-1-vinyl)thiazole 7a. Prepared from 2-[1-(hydroxyimino)ethyl]-thiazole S2a (2.4 g, $17.0 \mathrm{mmol})$; Yield: $37 \%$ (1.1 g, $6.2 \mathrm{mmol})$. Solvent system for flash chromatography: cyclohexane/EtOAc: $85 / 15$. Yellow solid; $\mathrm{Mp}: 45^{\circ} \mathrm{C} ;{ }^{1} \mathrm{H} \mathrm{NMR}\left(400 \mathrm{MHz}, \mathrm{CDCl}_{3}\right): \delta 8.45$ (br. s, $\left.1 \mathrm{H}\right)$, $7.70(\mathrm{~d}, J=3.3 \mathrm{~Hz}, 1 \mathrm{H}), 7.27(\mathrm{~d}, J=3.3 \mathrm{~Hz}, 1 \mathrm{H}), 6.42(\mathrm{~s}, 1 \mathrm{H}), 5.45(\mathrm{~s}, 1 \mathrm{H}), 2.19(\mathrm{~s}, 3 \mathrm{H}) ;{ }^{13} \mathrm{C} \mathrm{NMR}(100$ $\left.\mathrm{MHz}, \mathrm{CDCl}_{3}\right): \delta 169.0,165.8,142.2,134.2,119.8,101.7,25.0 ; \mathrm{IR}$ (ATR) $v_{\max } 3115,1681,1504,1373$, 1253, 1083, 851, 764, $676 \mathrm{~cm}^{-1}$; ESIHRMS $\mathrm{m} / \mathrm{z}$ calcd for $\mathrm{C}_{7} \mathrm{H}_{9} \mathrm{~N}_{2} \mathrm{OS}[\mathrm{M}+\mathrm{H}]^{+}$169.0430, found 169.0426 .

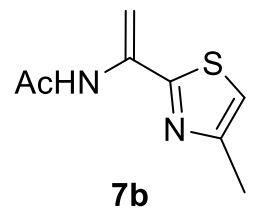

2-(Acetamido-1-vinyl)-4-methylthiazole 7b. Prepared from 2-[1-(hydroxyimino)ethyl]-4-methylthiazole S2b (1.1 g, $7.0 \mathrm{mmol}$ ); Yield: 45\% (570 mg, $3.13 \mathrm{mmol})$. Solvent system for flash

\footnotetext{
S2 Procedure adapted from: Hughes, R. A.; Thompson, S. P.; Alcaraz, L.; Moody, C. J. J. Am. Chem. Soc. 2005, 127, 15644.
} 
chromatography: cyclohexane/EtOAc: $85 / 15$. Beige solid. Mp: $34{ }^{\circ} \mathrm{C} ;{ }^{1} \mathrm{H} \mathrm{NMR}\left(400 \mathrm{MHz}, \mathrm{CDCl}_{3}\right): \delta$ 8.44 (br. s, 1H), 6.83-6.77 (m, 1H), 6.37 (s, 1H), 5.40 (s, 1H), 2.42 (d, J = 0.9 Hz, 3H), 2.19 (s, 3H);

${ }^{13} \mathrm{C} \mathrm{NMR}\left(100 \mathrm{MHz}, \mathrm{CDCl}_{3}\right): \delta$ 169.0, 164.7, 152.4, 134.3, 114.2, 101.0, 25.0, 17.1; IR (ATR) $v_{\max }$ 2924, 1694, 1514, 1444, 1368, 1081, 860, $649 \mathrm{~cm}^{-1}$; ESIHRMS $\mathrm{m} / z$ calcd for $\mathrm{C}_{8} \mathrm{H}_{11} \mathrm{~N}_{2} \mathrm{OS}[\mathrm{M}+\mathrm{H}]^{+}$ 183.0587, found 183.0581 .

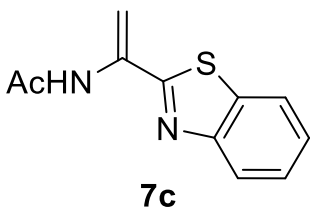

2-(Acetamido-1-vinyl)benzo[d]thiazole 7c. Prepared from 2-[1-(hydroxyimino)ethyl]benzo[d]thiazole S2c (604 mg, $2.58 \mathrm{mmol}$ ); Yield: 50\% (280 mg, $1.28 \mathrm{mmol})$. Solvent system for flash chromatography: cyclohexane/EtOAc: 80/20. Beige solid; Mp: $80{ }^{\circ} \mathrm{C} ;{ }^{1} \mathrm{H} \mathrm{NMR}(300 \mathrm{MHz}$, $\left.\mathrm{CDCl}_{3}\right): \delta 8.60(\mathrm{br} . \mathrm{s}, 1 \mathrm{H}), 8.00-7.94(\mathrm{~m}, 1 \mathrm{H}), 7.89-7.82(\mathrm{~m}, 1 \mathrm{H}), 7.53-7.38(\mathrm{~m}, 2 \mathrm{H}), 6.62$ (app. d, $J=1.0 \mathrm{~Hz}, 1 \mathrm{H}$ ), 5.59 (app. t, $J=1.4 \mathrm{~Hz}, 1 \mathrm{H}$ ), 2.25 (s, 3H); ${ }^{13} \mathrm{C} \mathrm{NMR}\left(75 \mathrm{MHz}, \mathrm{CDCl}_{3}\right.$ ): $\delta 169.1$, $165.4,152.5,134.9,134.5,126.7,126.2,123.3,121.9,104.7,25.1$; IR (ATR) $v_{\max } 2912,1715,1678$, 1520, 1495, 1434, 1375, 1257, 1087, 850, 750, $723 \mathrm{~cm}^{-1}$; ESIHRMS m/z calcd for $\mathrm{C}_{11} \mathrm{H}_{11} \mathrm{~N}_{2} \mathrm{OS}$ $[\mathrm{M}+\mathrm{H}]^{+} 219.0587$, found 219.0582 .

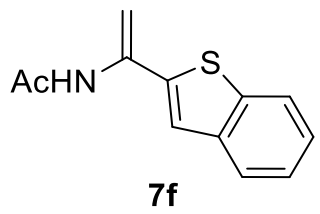

2-(Acetamido-1-vinyl)benzo[b]thiophene 7f. Prepared from 2-[1-(hydroxyimino)ethyl]benzo[b]thiophene S2f (2.9 g, $15.0 \mathrm{mmol}$ ); Yield: 38\% (1.2 g, $5.7 \mathrm{mmol})$. Solvent system for flash chromatography: cyclohexane/EtOAc: $75 / 25$. White solid; Mp: $138{ }^{\circ} \mathrm{C}$; ${ }^{1} \mathrm{HNMR}(600 \mathrm{MHz}$, DMSO- $\left.\mathrm{d}_{6}\right): \delta 9.48(\mathrm{~s}, 1 \mathrm{H}), 7.97-7.88(\mathrm{~m}, 1 \mathrm{H}), 7.83(\mathrm{dd}, J=6.4$ and $2.3 \mathrm{~Hz}, 1 \mathrm{H}), 7.60(\mathrm{~s}, 1 \mathrm{H}), 7.43-7.31$ $(\mathrm{m}, 2 \mathrm{H}), 5.71(\mathrm{~s}, 1 \mathrm{H}), 5.28(\mathrm{~s}, 1 \mathrm{H}), 2.09(\mathrm{~s}, 3 \mathrm{H}) ;{ }^{13} \mathrm{C}$ NMR (150 MHz, DMSO-d 6 ): $\delta$ 169.1, 141.0, 
139.6, 138.2, 135.4, 125.1, 124.8, 123.9, 122.3, 121.2, 104.4, 23.7; IR (ATR) $v_{\max } 2917,1669,1519$, $1374,1269,1033,864,726 \mathrm{~cm}^{-1}$; ESIHRMS $\mathrm{m} / \mathrm{z}$ calcd for $\mathrm{C}_{12} \mathrm{H}_{12} \mathrm{NOS}[\mathrm{M}+\mathrm{H}]^{+} 218.0634$, found 218.0630.

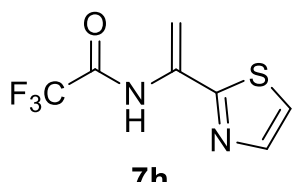

\section{2-(2,2,2-Trifluoroacetamido-1-vinyl)thiazole $7 \mathrm{~h}$.}

Trifluoroacetic anhydride $(4.0 \mathrm{~mL}, 28.8 \mathrm{mmol}, 3$ equiv.) and trifluoroacetic acid $(2.1 \mathrm{~mL}$, 28.8 mmol, 3 equiv.) were added to a solution of 2-[1-(hydroxyimino)ethyl]-thiazole S2a (1.4 g, $9.6 \mathrm{mmol}, 1$ equiv.) in freshly distilled ethyl acetate $(19 \mathrm{~mL})$ and the solution was purged with argon for 15 minutes. Iron ( $1.1 \mathrm{~g}, 19.2 \mathrm{mmol}, 2$ equiv.) was then added and the resulting mixture was stirred at $70{ }^{\circ} \mathrm{C}$ for 4 hours under argon. The reaction mixture was next cooled to room temperature and quenched with a saturated aqueous solution of $\mathrm{NaHCO}_{3}$ and extracted thrice with ethyl acetate. The combined organic layers were successively washed with a saturated aqueous solution of $\mathrm{NaHCO}_{3}$ and brine, dried over magnesium sulfate, filtered and concentrated. The crude residue was finally purified by flash column chromatography over silica gel (cyclohexane/EtOAc: 95/5) to yield the desired thiazole $7 \mathrm{~h}$ as a beige solid (668 $\mathrm{mg}, 3.01 \mathrm{mmol}$, 31\%). Mp: $33^{\circ} \mathrm{C} ;{ }^{1} \mathrm{H} \mathrm{NMR} \mathrm{(400} \mathrm{MHz,} \mathrm{CDCl}_{3}$ ): $\delta 9.43$ (br. s, $1 \mathrm{H}$ ), 7.75 (d, J = $3.2 \mathrm{~Hz}, 1 \mathrm{H}$ ), 7.34 (d, $J=3.3 \mathrm{~Hz}, 1 \mathrm{H}$ ), 6.51 (app. d, $J=2.0 \mathrm{~Hz}, 1 \mathrm{H}), 5.67$ (app. t, $J=1.7 \mathrm{~Hz}, 1 \mathrm{H}) ;{ }^{13} \mathrm{C} \mathrm{NMR}\left(100 \mathrm{MHz}, \mathrm{CDCl}_{3}\right)$ : $\delta 163.7,155.3\left(q, J_{C-F}=37.8 \mathrm{~Hz}\right), 142.5,132.7,120.6,115.6\left(q, J_{C-F}=288.7 \mathrm{~Hz}\right), 104.6$; ${ }^{19} \mathrm{~F} N M R$ (376 MHz, $\mathrm{CDCl}_{3}$ ): $\delta-76.5$ (s); IR (ATR) $v_{\max }$ 1729, 1541, 1488, 1203, 1159, 910, 736, $660 \mathrm{~cm}^{-1}$; ESIHRMS $m / z$ calcd for $\mathrm{C}_{7} \mathrm{H}_{6} \mathrm{~F}_{3} \mathrm{~N}_{2} \mathrm{OS}[\mathrm{M}+\mathrm{H}]^{+} 223.0147$, found 223.0141 . 


\section{Experimental Procedures and Characterization Data: Enantioselective Hydrogenation of 2-Enamido-heteroarenes}

\section{General procedure:}

In a glovebox, the starting 2-enamido-heteroarene $(2 \mathrm{mmol}, 1$ equiv.), bis(1,5cyclooctadiene)rhodium(I) tetrafluoroborate (16 mg, $0.04 \mathrm{mmol}, 2 \mathrm{~mol} \%)$ and (S,S)-2,3-bis(tertbutylmethyl-phosphino)quinoxaline (17 mg, $0.05 \mathrm{mmol}, 2.5 \mathrm{~mol} \%$ ) were placed into a $25 \mathrm{~mL}$ flask that was next closed with a rubber septum and taken outside of the glovebox. Dry and freshly degassed methanol $(20 \mathrm{~mL})$ was next added under argon before quickly introducing the reaction mixture in an autoclave. The autoclave was then pressurized at 10 bar with $\mathrm{H}_{2}$ and the reaction mixture was stirred overnight at $25^{\circ} \mathrm{C}$. After releasing the pressure, the solvent was evaporated under reduced pressure and the crude residue was finally purified by flash column chromatography over silica gel.

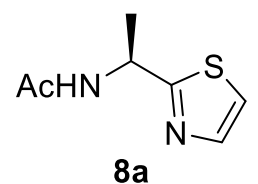

(S)-2-(Acetamidoethyl)thiazole 8a. Prepared from 2-(acetamido-1-vinyl)thiazole 7a (336 mg, 2.00 mmol); Yield: 90\% (307 mg, $1.80 \mathrm{mmol}$ ) and 95\% ee. The ee was determined by comparison of HPLC analyses of the optically enriched product and the racemic one (Daicel Chiralpak ADH, $n$-hexane/isopropanol: 93/7, flow rate: $0.4 \mathrm{~mL} \cdot \mathrm{min}^{-1}, 238 \mathrm{~nm}, \mathrm{t}_{R}=9.0 \mathrm{~min}, \mathrm{t}_{\mathrm{s}}=10.1 \mathrm{~min}$ ). $[\alpha]_{\mathrm{D}}^{20}-111^{\circ} \cdot \mathrm{dm}^{-1} \cdot \mathrm{g}^{-1} \cdot \mathrm{cm}^{3}\left(\mathrm{c} 0.83, \mathrm{CHCl}_{3}\right)$. Solvent system for flash chromatography: EtOAc. White solid; ${ }^{1} \mathrm{H}$ NMR (400 MHz, $\left.\mathrm{CDCl}_{3}\right): \delta 7.63(\mathrm{~d}, J=3.1 \mathrm{~Hz}, 1 \mathrm{H}), 7.21$ (d, J=3.1 Hz, $1 \mathrm{H}$ ), 6.93 (br. s, $\left.1 \mathrm{H}\right)$, 5.36 (quint., $J=7.0 \mathrm{~Hz}, 1 \mathrm{H}), 1.96(\mathrm{~s}, 3 \mathrm{H}), 1.54(\mathrm{~d}, J=6.9 \mathrm{~Hz}, 3 \mathrm{H}) ;{ }^{13} \mathrm{C} \mathrm{NMR}\left(100 \mathrm{MHz}, \mathrm{CDCl}_{3}\right): \delta$ $172.5,169.6,142.2,119.0,47.0,23.1,21.7$. This compound has been previously partially described. ${ }^{S 2}$ 


\section{HPLC trace of (S)-8a:}

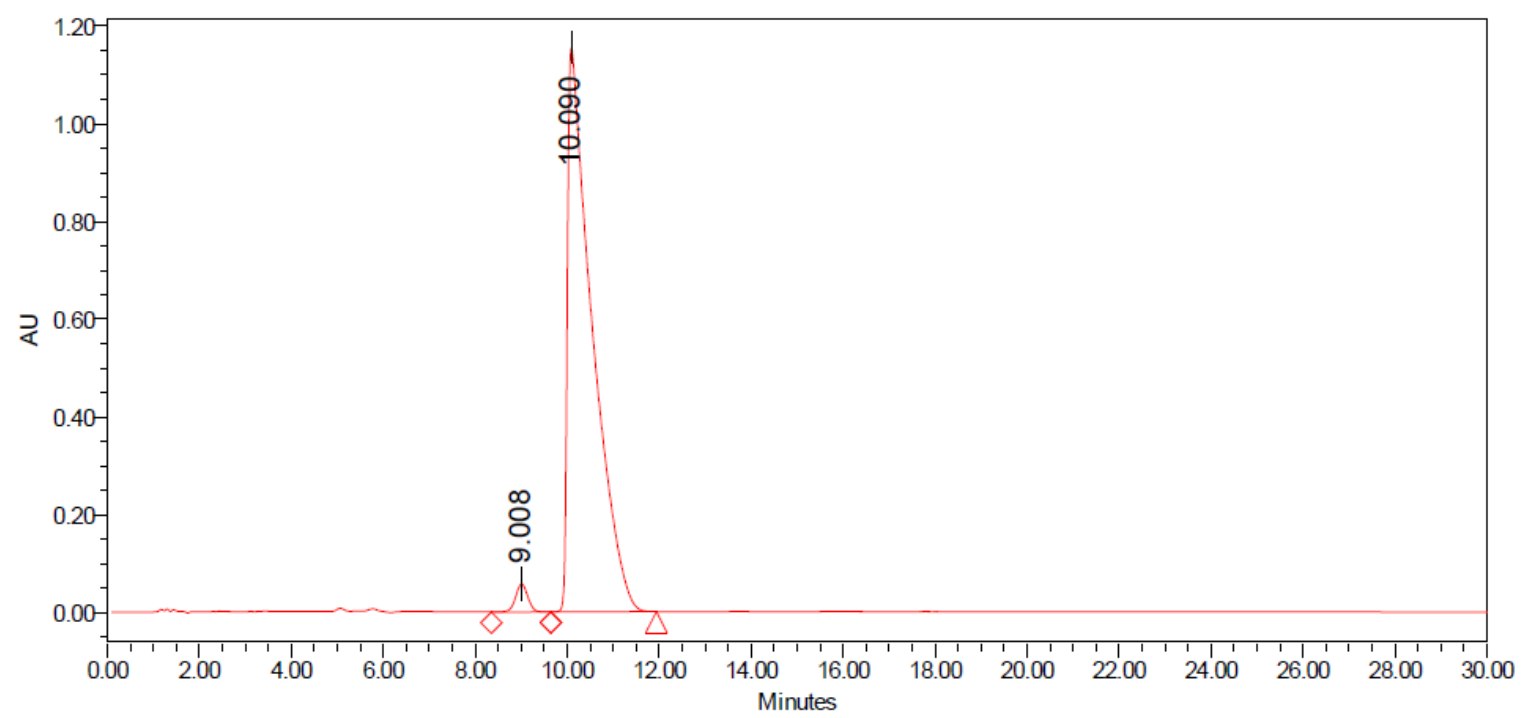

Peak Results
\begin{tabular}{|r|c|c|c|r|r|r|r|}
\hline & Name & RT & Area & Height & Units & $\%$ Area & $\%$ Height \\
\hline 1 & & 9.008 & 1083534 & 57493 & & 2.46 & 4.74 \\
\hline 2 & & 10.090 & 42895081 & 115471 & & 97.54 & 95.26 \\
\hline
\end{tabular}

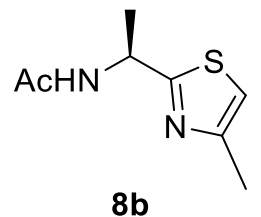

(S)-2-(Acetamidoethyl)-4-methylthiazole 8b. Prepared from 2-(acetamido-1-vinyl)-4-methyl thiazole 7b (361 mg, $2.00 \mathrm{mmol})$; Yield: 86\% (318 mg, $1.73 \mathrm{mmol}$ ) and 96\% ee. The ee was determined by comparison of HPLC analyses of the optically enriched product and the racemic one (Daicel Chiralpak ADH, $n$-hexane/isopropanol: 95/5, flow rate: $0.4 \mathrm{~mL}$. $\mathrm{min}^{-1}, 238 \mathrm{~nm}, \mathrm{t}_{s}=11.1$ $\min , t_{R}=13.0 \mathrm{~min}$ ). $[\alpha]_{\mathrm{D}}^{20}-89^{\circ} \cdot \mathrm{dm}^{-1} \cdot \mathrm{g}^{-1} \cdot \mathrm{cm}^{3}$ (c $0.83, \mathrm{CHCl}_{3}$ ). Solvent system for flash chromatography: EtOAc. Yellow solid; $\mathrm{Mp}: 86^{\circ} \mathrm{C} ;{ }^{1} \mathrm{H}$ NMR $\left(400 \mathrm{MHz}, \mathrm{CDCl}_{3}\right): \delta 6.82-6.72(\mathrm{~m}, 1 \mathrm{H})$, 6.44 (br. s, 1H), 5.34 (quint., $J=6.9 \mathrm{~Hz}, 1 \mathrm{H}$ ), 2.41 (d, J = $0.8 \mathrm{~Hz}, 3 \mathrm{H}$ ), 2.02 (s, 3H), 1.57 (d, J = $6.9 \mathrm{~Hz}$, $3 \mathrm{H}) ;{ }^{13} \mathrm{C}$ NMR $\left(100 \mathrm{MHz}, \mathrm{CDCl}_{3}\right): \delta 171.4,169.4,152.6,113.3,47.3,23.4,22.4,17.1$; IR (ATR) $v_{\max }$ 2952, 1710, 1558, 1189, 1146, 1054, 723, $655 \mathrm{~cm}^{-1}$; ESIHRMS $\mathrm{m} / z$ calcd for $\mathrm{C}_{8} \mathrm{H}_{13} \mathrm{~N}_{2} \mathrm{OS}$ $[\mathrm{M}+\mathrm{H}]^{+}$185.0743, found 185.0739 . 


\section{HPLC trace of (S)-8b:}

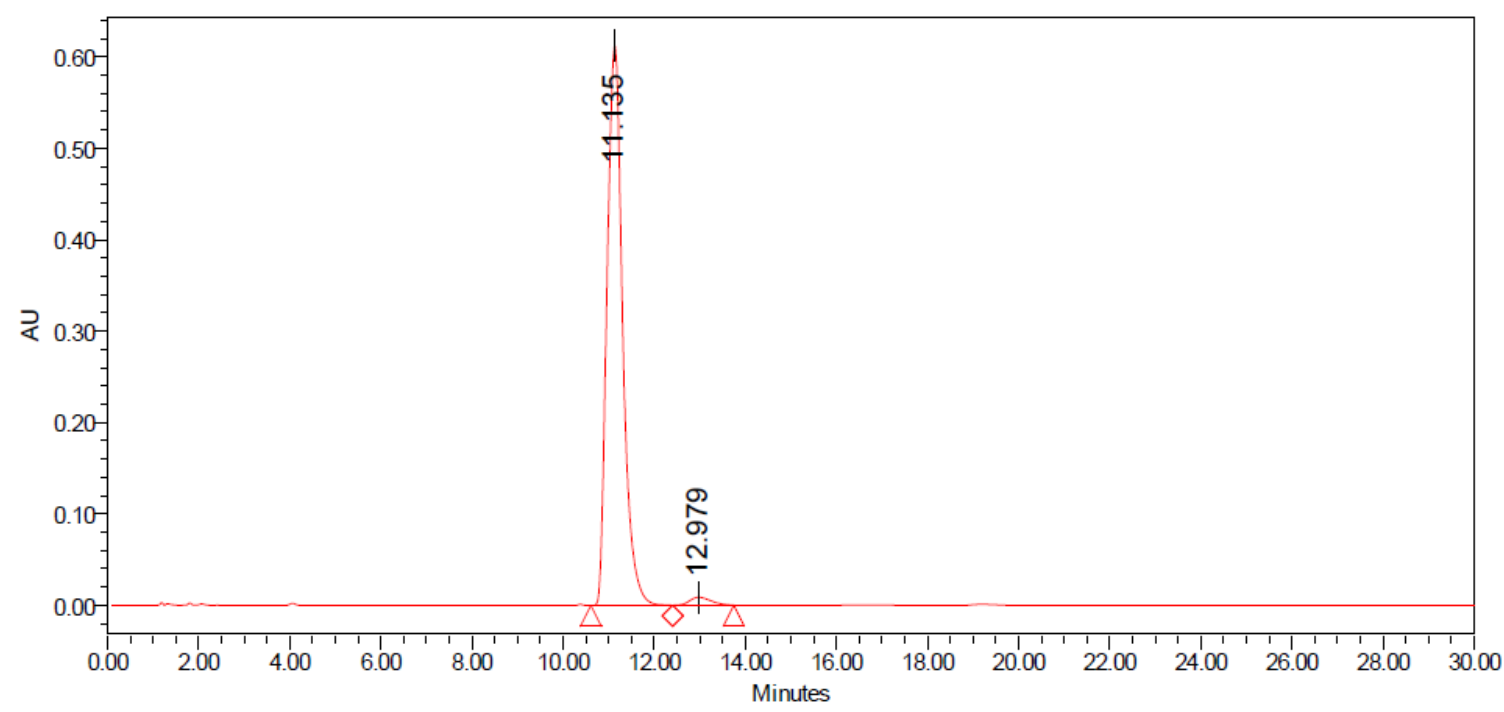

Peak Results
\begin{tabular}{|r|c|c|r|r|r|r|r|}
\hline & Name & RT & Area & Height & Units & $\%$ Area & $\%$ Height \\
\hline 1 & & 11.135 & 14575094 & $61196 \mathrm{C}$ & & 98.11 & 98.60 \\
\hline 2 & & 12.979 & 281498 & 8687 & & 1.89 & 1.40 \\
\hline
\end{tabular}

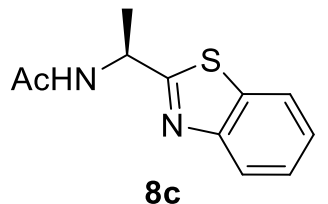

(S)-2-(Acetamidoethyl)benzo[d]thiazole 8c. Prepared from 2-(acetamido-1-vinyl) benzo[d]thiazole 7c (238 mg, $1.10 \mathrm{mmol}$ ); Yield: 76\% (182 mg, $0.83 \mathrm{mmol}$ ) and 94\% ee. The ee was determined by comparison of HPLC analyses of the optically enriched product and the racemic one (Daicel Chiralpak $A D H, n$-hexane/isopropanol: 93/7, flow rate: $0.4 \mathrm{~mL}^{\mathrm{min}}{ }^{-1}, 238 \mathrm{~nm}$, $\left.\mathrm{t}_{\mathrm{s}}=10.0 \mathrm{~min}, \mathrm{t}_{R}=13.4 \mathrm{~min}\right) \cdot[\alpha]_{\mathrm{D}}^{20}-100^{\circ} \cdot \mathrm{dm}^{-1} \cdot \mathrm{g}^{-1} \cdot \mathrm{cm}^{3}(c 0.83, \mathrm{MeOH}) .^{\mathrm{S} 3}$ Solvent system for flash chromatography: EtOAc. Yellow solid; ${ }^{1} \mathrm{H}$ NMR (400 MHz, $\left.\mathrm{CDCl}_{3}\right): \delta 7.97(\mathrm{~d}, J=8.2 \mathrm{~Hz}, 1 \mathrm{H}), 7.85$ $(\mathrm{d}, J=7.9 \mathrm{~Hz}, 1 \mathrm{H}), 7.54-7.42(\mathrm{~m}, 1 \mathrm{H}), 7.42-7.32(\mathrm{~m}, 1 \mathrm{H}), 6.60(\mathrm{~d}, J=6.0 \mathrm{~Hz}, 1 \mathrm{H}), 5.49$ (quint., $J=7.0 \mathrm{~Hz}, 1 \mathrm{H}), 2.07(\mathrm{~s}, 3 \mathrm{H}), 1.67(\mathrm{~d}, J=6.9 \mathrm{~Hz}, 3 \mathrm{H}) ;{ }^{13} \mathrm{C} \mathrm{NMR}\left(100 \mathrm{MHz}, \mathrm{CDCl}_{3}\right): \delta$ 173.0, 169.6,

\footnotetext{
S3 In agreement with the literature: $[\alpha]_{\mathrm{D}, \mathrm{Litt}}^{25}(R)+119.6^{\circ} \cdot \mathrm{dm}^{-1} \cdot \mathrm{g}^{-1} \cdot \mathrm{cm}^{3}(c \mathrm{1} \cdot 0, \mathrm{MeOH}, 99 \% \text { ee })^{\mathrm{s}}$
} 
$152.9,135.1,126.3,125.4,123.0,121.9,48.0,23.4,22.1$. This compound has been previously reported. ${ }^{S 4}$

HPLC trace of (S)-8c:

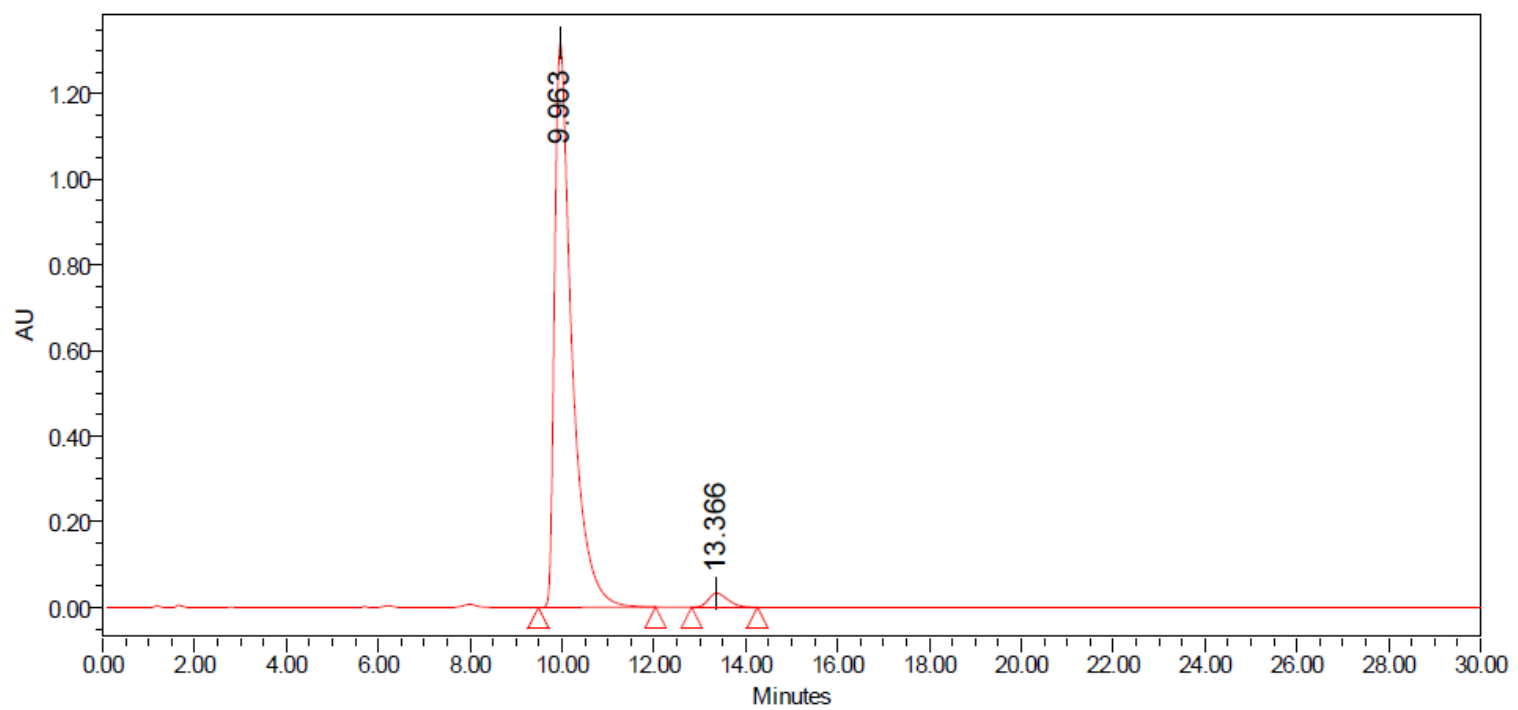

Peak Results

\begin{tabular}{|r|c|c|r|r|r|r|r|}
\hline & Name & \multicolumn{1}{c|}{ RT } & \multicolumn{1}{c|}{ Area } & Height & Units & \% Area & \% Height \\
\hline 1 & & 9.963 & 34546126 & 1317801 & & 97.30 & 97.59 \\
\hline 2 & & 13.366 & 958396 & 32579 & & 2.70 & 2.41 \\
\hline
\end{tabular}

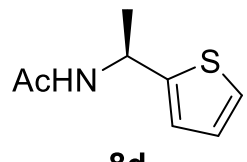

$8 d$

(S)-2-(Acetamidoethyl)thiophene 8d. Prepared from 2-(acetamido-1-vinyl)thiophene 7d (335 $\mathrm{mg}, 2.00 \mathrm{mmol}$ ); Yield: 92\% (312 $\mathrm{mg}, 1.85 \mathrm{mmol}$ ) and 97\% ee. The ee was determined by comparison of HPLC analyses of the optically enriched product and the racemic one (Daicel Chiralpak ADH, $n$-hexane/isopropanol: 95/5, flow rate: $0.4 \mathrm{~mL} \cdot \mathrm{min}^{-1}, 231.8 \mathrm{~nm}, \mathrm{t}_{R}=8.8 \mathrm{~min}$, $\left.\mathrm{t}_{s}=10.7 \mathrm{~min}\right) . \quad[\alpha]_{\mathrm{D}}^{20}-133{ }^{\circ} \cdot \mathrm{dm}^{-1} \cdot \mathrm{g}^{-1} \cdot \mathrm{cm}^{3} \quad\left(\mathrm{c} \quad 0.83, \mathrm{CHCl}_{3}\right) .{ }^{\mathrm{S5}}$ Solvent system for flash chromatography: cyclohexane/EtOAc: 50/50. Yellow solid; ${ }^{1} \mathrm{H} \mathrm{NMR}\left(400 \mathrm{MHz}, \mathrm{CDCl}_{3}\right): \delta 7.19$ (app.

\footnotetext{
${ }^{54}$ Alatorre- Santamaría, S.; Gotor-Fernández, V.; Gotor, V. Eur. J. Org. Chem. 2009, 15, 2533

${ }^{55}$ In agreement with the literature $[\alpha]_{\mathrm{D}, \mathrm{Litt}}^{25}-127^{\circ} \cdot \mathrm{dm}^{-1} \cdot \mathrm{g}^{-1} \cdot \mathrm{cm}^{3}\left(\mathrm{c} 1.0, \mathrm{CHCl}_{3},>99 \%\right.$ ee $\left.(\mathrm{S})\right) .{ }^{\mathrm{S} 6}$
} 
$\mathrm{dd}, J=4.9$ and $1.4 \mathrm{~Hz}, 1 \mathrm{H}$ ), 6.98-6.92 (m, 2H), 5.87 (br. s, $1 \mathrm{H}$ ), 5.39 (quint., $J=7.2 \mathrm{~Hz}, 1 \mathrm{H}$ ), 1.97 (s, 3H), $1.56(\mathrm{~d}, J=6.8 \mathrm{~Hz}, 3 \mathrm{H}) ;{ }^{13} \mathrm{C}$ NMR $\left(100 \mathrm{MHz}, \mathrm{CDCl}_{3}\right): \delta 169.1,147.2,126.9,124.4,124.1,44.7$, 23.5, 22.3. This compound has been previously partially described. ${ }^{\mathrm{S6}}$

HPLC trace of (S)-8d:

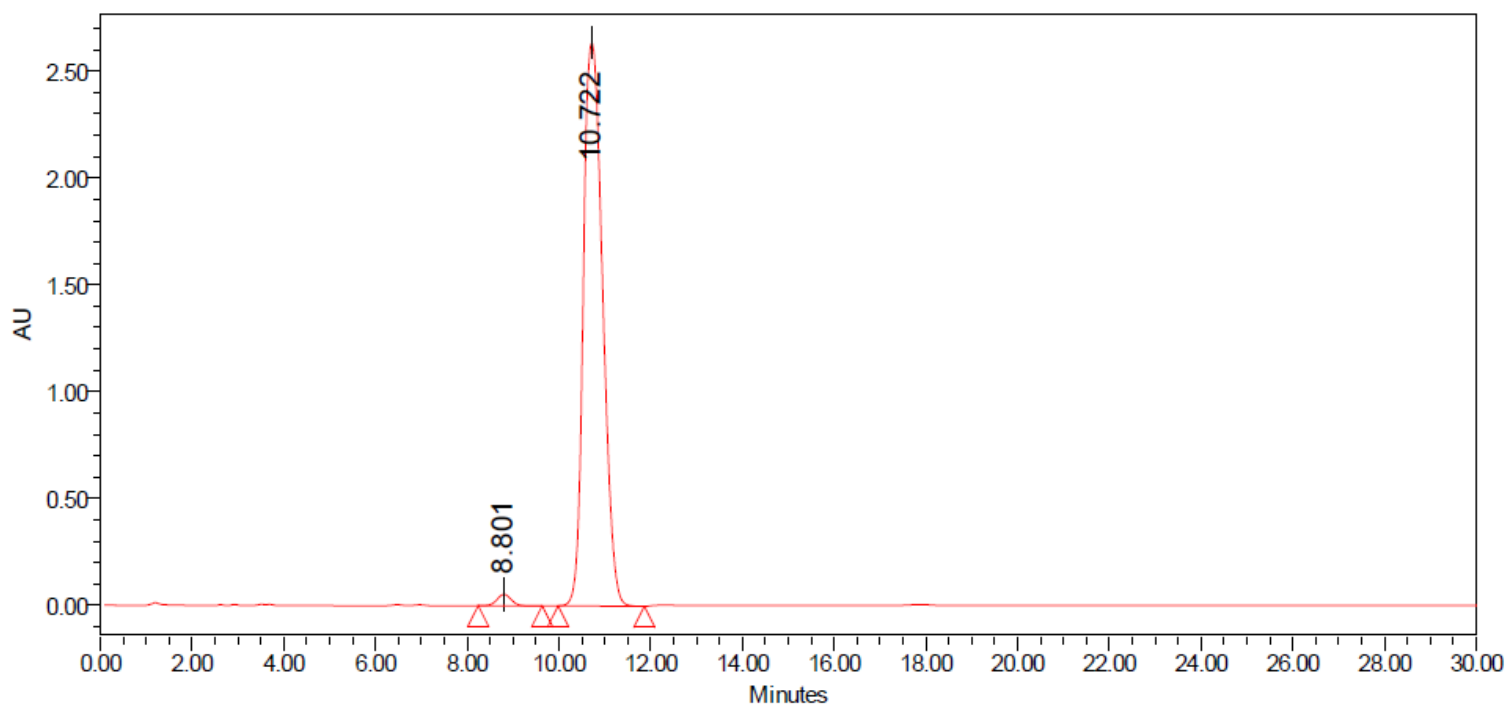

Peak Results
\begin{tabular}{|r|r|r|r|r|r|r|r|}
\hline & Name & \multicolumn{1}{c|}{ RT } & Area & Height & Units & $\%$ Area & $\%$ Height \\
\hline 1 & & 8.801 & 1138094 & 53198 & & 1.46 & 1.98 \\
\hline 2 & & 10.722 & 76646165 & 2634408 & & 98.54 & 98.02 \\
\hline
\end{tabular}

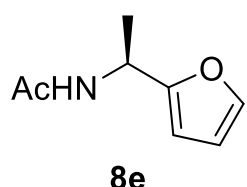

(S)-2-(Acetamidoethyl)furan 8e. Prepared from 2-(acetamido-1-vinyl)furan 7e (302 mg, 2.00 mmol); Yield: 86\% (265 mg, $1.75 \mathrm{mmol}$ ) and 98\% ee. The ee was determined by comparison of HPLC analyses of the optically enriched product and the racemic one (Daicel Chiralpak ADH, $n$-hexane/isopropanol: 95/5, flow rate: $0.4 \mathrm{~mL} \cdot \mathrm{min}^{-1}, 210 \mathrm{~nm}, \mathrm{t}_{R}=12.2 \mathrm{~min}, \mathrm{t}_{\mathrm{s}}=14.2$

S6 Mohar, B.; Stephan, M. Adv. Synth. Catal. 2013, 355, 594. 
min). $[\alpha]_{\mathrm{D}}^{20}-153^{\circ} \cdot \mathrm{dm}^{-1} \cdot \mathrm{g}^{-1} \cdot \mathrm{cm}^{3}$ (c $0.83, \mathrm{CHCl}_{3}$ ). Solvent system for flash chromatography: cyclohexane/EtOAc: 40/60. Beige solid. This compound has been previously reported. ${ }^{S 7}$

HPLC trace of (S)-8e:

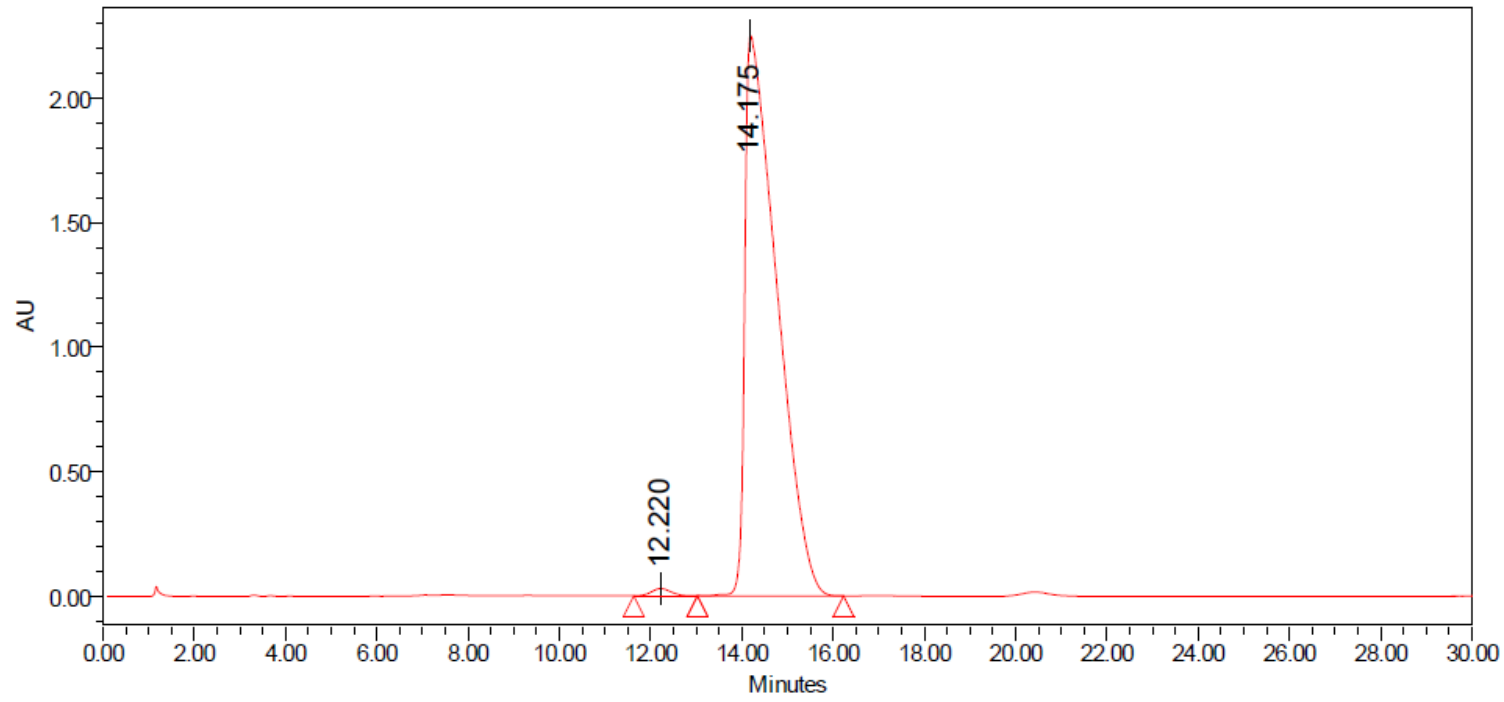

Peak Results

\begin{tabular}{|r|c|c|r|r|r|r|r|}
\hline & Name & RT & Area & Height & Units & \% Area & \% Height \\
\hline 1 & & 12.220 & 875043 & 29289 & & 0.79 & 1.29 \\
\hline 2 & & 14.175 & 109679032 & 2248130 & & 99.21 & 98.71 \\
\hline
\end{tabular}

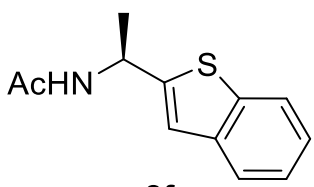

$8 f$

(S)-2-(Acetamidoethyl)benzo[b]thiophene 8f. Prepared from 2-(acetamido-1-vinyl) benzo[b]thiophene $7 f$ (432 mg, $2.00 \mathrm{mmol}$ ); Yield: 94\% (413 mg, $1.89 \mathrm{mmol}$ ) and 87\% ee. The ee was determined by comparison of HPLC analyses of the optically enriched product and the racemic one (Daicel Chiralpak ADH, $n$-hexane/isopropanol: 93/7, flow rate: $0.4 \mathrm{~mL} . \mathrm{min}^{-1}, 238$ $\left.\mathrm{nm}, \mathrm{t}_{R}=5.2 \mathrm{~min}, \mathrm{t}_{s}=7.1 \mathrm{~min}\right) \cdot[\alpha]_{\mathrm{D}}^{20}-128^{\circ} \cdot \mathrm{dm}^{-1} \cdot \mathrm{g}^{-1} \cdot \mathrm{cm}^{3}\left(\mathrm{c} 0.83, \mathrm{CHCl}_{3}\right)$. Solvent system for flash chromatography: cyclohexane/EtOAc: 50/50. White solid; Mp: $133{ }^{\circ} \mathrm{C}$; ${ }^{1} \mathrm{H} \mathrm{NMR}(400 \mathrm{MHz}$,

S7 Blume, F.; Albeiruty, M.; Deska, J. Synthesis 2015, 47, 2093. 
$\left.\mathrm{CDCl}_{3}\right): \delta 7.77(\mathrm{~d}, J=7.8 \mathrm{~Hz}, 1 \mathrm{H}), 7.69(\mathrm{dd}, J=7.2$ and $1.4 \mathrm{~Hz}, 1 \mathrm{H}), 7.37-7.24(\mathrm{~m}, 2 \mathrm{H}), 7.16(\mathrm{~s}, 1 \mathrm{H})$, 6.13 (br. d, $J=6.5 \mathrm{~Hz}, 1 \mathrm{H}$ ), 5.45 (quint., $J=7.3 \mathrm{~Hz}, 1 \mathrm{H}$ ), $2.00(\mathrm{~s}, 3 \mathrm{H}), 1.61(\mathrm{~d}, J=6.9 \mathrm{~Hz}, 3 \mathrm{H}$ ); ${ }^{13} \mathrm{C}$ NMR $\left(100 \mathrm{MHz}_{2} \mathrm{CDCl}_{3}\right): \delta 169.3,147.9,139.6,139.2,124.5,124.3,123.5,122.4,120.5,45.2$, 23.4, 21.9; IR (ATR) $v_{\max } 2975,1634,1555,1438,1371,1302,1170,840,760,657 \mathrm{~cm}^{-1}$; ESIHRMS $m / z$ calcd for $\mathrm{C}_{12} \mathrm{H}_{14} \mathrm{NOS}[\mathrm{M}+\mathrm{H}]^{+} 220.0791$, found 220.0787 .

\section{HPLC trace of (S)-8f:}

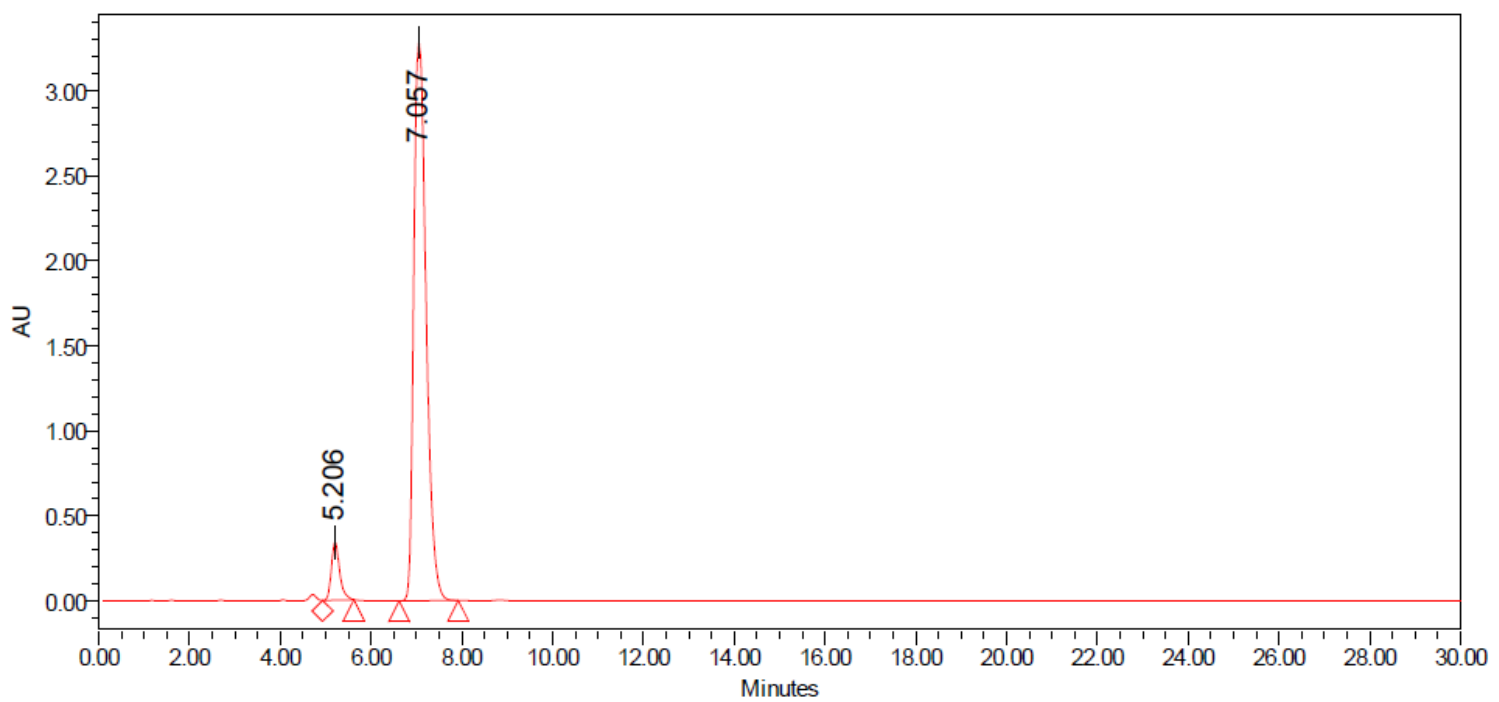

Peak Results

\begin{tabular}{|r|c|c|c|r|r|r|r|}
\hline & Name & RT & Area & Height & Units & \% Area & $\%$ Height \\
\hline 1 & & 5.206 & 4329522 & 339128 & & 6.53 & 9.37 \\
\hline 2 & & 7.057 & 61983592 & 3279893 & & 93.47 & 90.63 \\
\hline
\end{tabular}

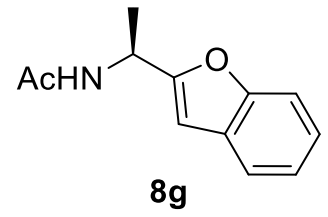

(S)-2-(Acetamidoethyl)benzofuran 8g. Prepared from 2-(acetamido-1-vinyl)benzofuran 7g (402 $\mathrm{mg}, 2.00 \mathrm{mmol}$ ); Yield: 88\% (360 mg, $1.77 \mathrm{mmol}$ ) and >99\% ee. The ee was determined by comparison of HPLC analyses of the optically enriched product and the racemic one (Daicel Chiralpak ADH, $n$-hexane/isopropanol: 95/5, flow rate: $0.4 \mathrm{~mL} \cdot \mathrm{min}^{-1}, 238 \mathrm{~nm}, \mathrm{t}_{s}=4.6 \mathrm{~min}, \mathrm{t}_{R}=5.4$ min). $[\alpha]_{D}^{20}-217^{\circ} \cdot \mathrm{dm}^{-1} \cdot \mathrm{g}^{-1} \cdot \mathrm{cm}^{3}$ (c $0.83, \mathrm{CHCl}_{3}$ ). Solvent system for flash chromatography: 
cyclohexane/EtOAc: 50/50. Yellow solid; Mp: $138{ }^{\circ} \mathrm{C} ;{ }^{1} \mathrm{H} \mathrm{NMR}\left(400 \mathrm{MHz}, \mathrm{CDCl}_{3}\right): \delta 7.53-7.48(\mathrm{~m}$, $1 \mathrm{H}), 7.43(\mathrm{~d}, J=7.9 \mathrm{~Hz}, 1 \mathrm{H}), 7.28-7.16(\mathrm{~m}, 3 \mathrm{H}), 6.55(\mathrm{~s}, 1 \mathrm{H}), 6.03$ (br. $\mathrm{d}, J=7.1 \mathrm{~Hz}, 1 \mathrm{H}), 5.34$ (quint., $J=7.0 \mathrm{~Hz}, 1 \mathrm{H}), 2.00(\mathrm{~s}, 3 \mathrm{H}), 1.55(\mathrm{~d}, J=7.0 \mathrm{~Hz}, 3 \mathrm{H}) ;{ }^{13} \mathrm{C} \mathrm{NMR}\left(100 \mathrm{MHz}, \mathrm{CDCl}_{3}\right): \delta$ 169.3, 158.1, $154.8,128.2,124.2,122.9,121.1,111.2,102.5,43.4,23.4,19.8$; IR (ATR) $v_{\max } 2945,1644,1533$, $1455,1368,1304,1162,816,763,720 \mathrm{~cm}^{-1}$; ESIHRMS m/z calcd for $\mathrm{C}_{12} \mathrm{H}_{14} \mathrm{NO}_{2}[\mathrm{M}+\mathrm{H}]^{+} 204.1019$; found 204.1014.

HPLC trace of (S)-8g:

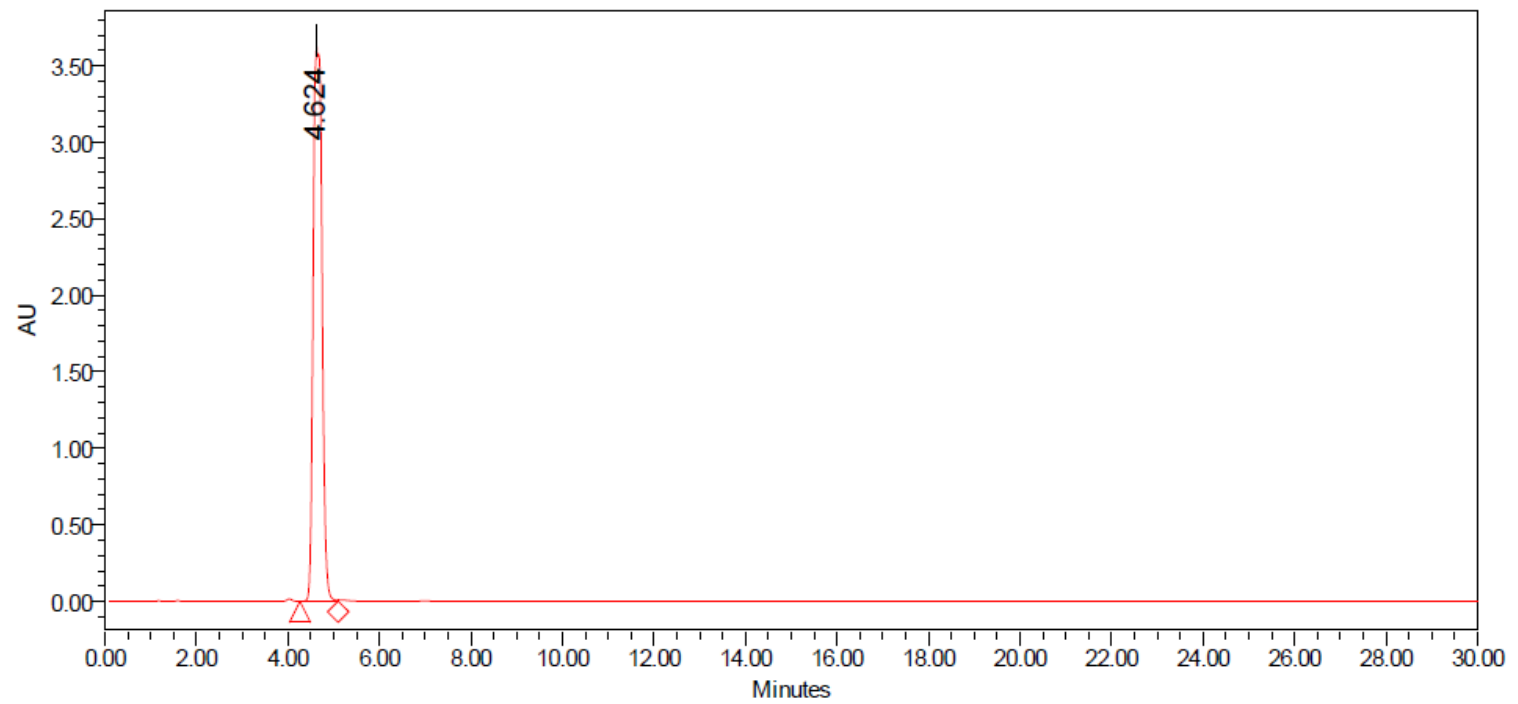<smiles>C[C@H](NC(=O)C(F)(F)F)c1nccs1</smiles>

$8 \mathrm{~h}$

(S)-2-(2,2,2-Trifluoroacetamidoethyl)thiazole 8h. Prepared from 2-(2,2,2-trifluoroacetamido-1vinyl)thiazole $7 \mathrm{~h}$ (317 mg, $1.40 \mathrm{mmol}$ ); Yield: 84\% (272 mg, $1.21 \mathrm{mmol}$ ) and 98\% ee. The ee was determined by comparison of HPLC analyses of the optically enriched product and the racemic one (Daicel Chiralpak ADH, $n$-hexane/isopropanol: 95/5, flow rate: $0.4 \mathrm{~mL} \cdot \mathrm{min}^{-1}, 238.8 \mathrm{~nm}, \mathrm{t}_{S}=$ $\left.8.1 \mathrm{~min}, \mathrm{t}_{R}=9.5 \mathrm{~min}\right) .[\alpha]_{\mathrm{D}}^{20}-77^{\circ} \cdot \mathrm{dm}^{-1} \cdot \mathrm{g}^{-1} \cdot \mathrm{cm}^{3}$ (c $\left.0.83, \mathrm{CHCl}_{3}\right)$. Solvent system for flash chromatography: cyclohexane/EtOAc: $75 / 25$. Beige solid; $\mathrm{Mp}: 73{ }^{\circ} \mathrm{C} ;{ }^{1} \mathrm{H} \mathrm{NMR}\left(300 \mathrm{MHz}, \mathrm{CDCl}_{3}\right): \delta$ 7.96 (br. s, 1H), 7.69 (d, J = 3.3 Hz, 1H), 7.31 (d, J=3.3 Hz, 1H), 5.40 (quint., $J=7.0 \mathrm{~Hz}, 1 \mathrm{H}$ ), 1.66 
$(\mathrm{d}, J=6.9 \mathrm{~Hz}, 3 \mathrm{H}) ;{ }^{13} \mathrm{C}$ NMR $\left(75 \mathrm{MHz}, \mathrm{CDCl}_{3}\right): \delta 169.5,156.7\left(\mathrm{q}, J_{C-F}=37.6 \mathrm{~Hz}\right), 142.3,119.8,115.8$ $\left(q, J_{C-F}=287.7 \mathrm{~Hz}\right), 47.9,21.7 ;{ }^{19} \mathrm{~F}$ NMR $\left(376 \mathrm{MHz}, \mathrm{CDCl}_{3}\right): \delta-76.3$ (s); IR (ATR) $v_{\max } 2952,1710$, $1558,1189,1146,1054,758,723,655 \mathrm{~cm}^{-1}$; ESIHRMS $\mathrm{m} / \mathrm{z}$ calcd for $\mathrm{C}_{7} \mathrm{H}_{8} \mathrm{~F}_{3} \mathrm{~N}_{2} \mathrm{OS}[\mathrm{M}+\mathrm{H}]^{+} 225.0304$, found 225.0300 .

HPLC trace of (S)-8h:

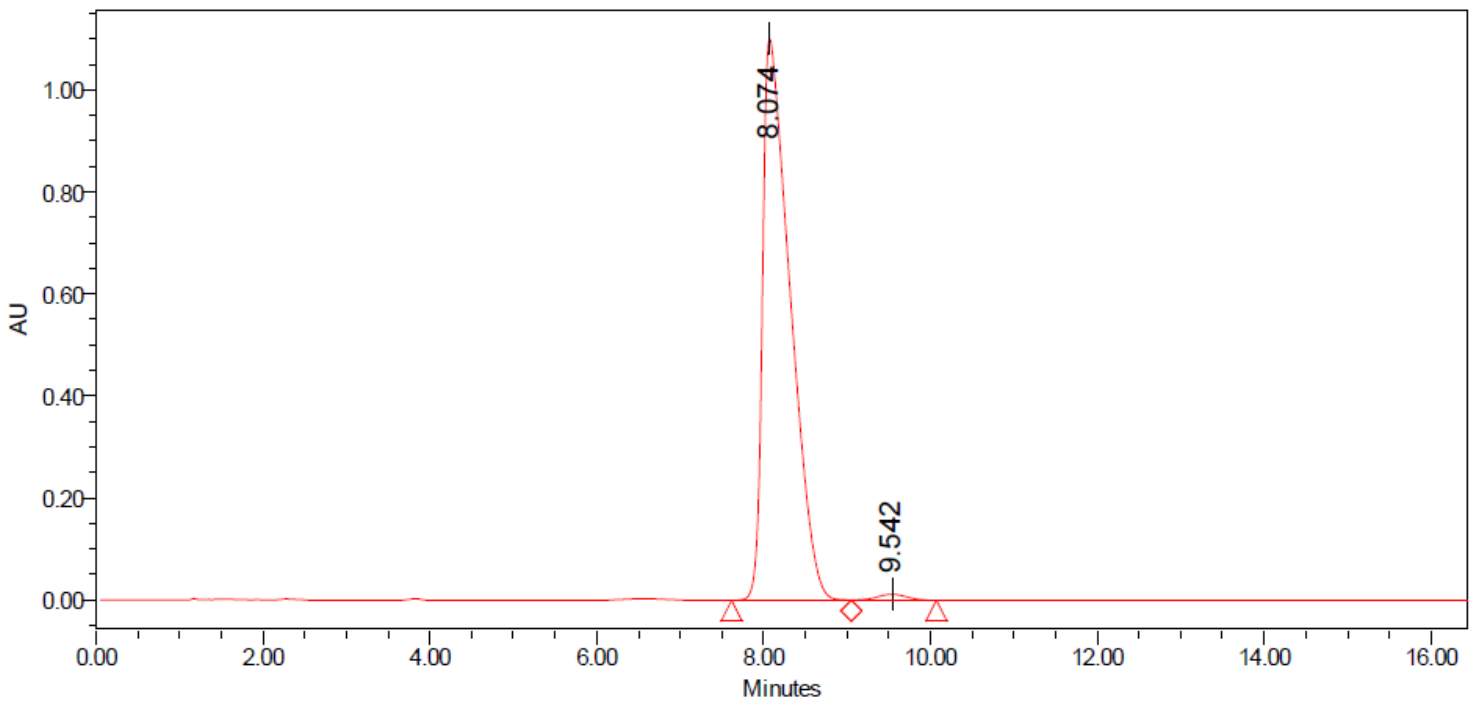

Peak Results

\begin{tabular}{|r|c|c|r|r|r|r|r|}
\hline & Name & RT & \multicolumn{1}{c|}{ Area } & Height & Units & \% Area & \% Height \\
\hline 1 & & 8.074 & 25275544 & $110029 \subseteq$ & & 98.90 & 98.96 \\
\hline 2 & & 9.542 & 281269 & 11572 & & 1.10 & 1.04 \\
\hline
\end{tabular}<smiles>C[C@H](N)c1nccs1</smiles>

$9 \cdot \mathrm{HCl}$

\section{(S)-2-(1-aminoethyl)thiazole hydrochloride $9 \cdot \mathrm{HCl}$.}

(S)-2-(2,2,2-Trifluoroacetamidoethyl)thiazole $8 \mathrm{~h}(56 \mathrm{mg}, 0.25 \mathrm{mmol})$ and potassium carbonate (4 equiv.) were dissolved in $\mathrm{MeOH} / \mathrm{H}_{2} \mathrm{O}(10 / 1 \mathrm{v} / \mathrm{v}, 4.4 \mathrm{~mL})$ in a pressure tube. The resulting solution was stirred at $60^{\circ} \mathrm{C}$ for 48 hours, then cooled to room temperature and next partitioned between dichloromethane and water. The aqueous layer was extracted twice with dichloromethane and the combined organic layers were then extracted with a $1 \mathrm{M}$ aqueous solution of $\mathrm{HCl}$. The aqueous 
layer was finally concentrated under reduced pressure and the residue was dried under high vacuum to give the desired (S)-2-(1-aminoethyl)thiazole hydrochloride as a white solid (41.2 $\mathrm{mg}$, 0.25 mmol, quant.). $[\alpha]_{\mathrm{D}}^{20}-6^{\circ} \cdot \mathrm{dm}^{-1} \cdot \mathrm{g}^{-1} \cdot \mathrm{cm}^{3}\left(c 0.83, \mathrm{H}_{2} \mathrm{O}\right) \cdot{ }^{1} \mathrm{H} \mathrm{NMR}\left(400 \mathrm{MHz}, \mathrm{D}_{2} \mathrm{O}\right): \delta 7.87(\mathrm{~d}, J=$ $3.4 \mathrm{~Hz}, 1 \mathrm{H}), 7.75(\mathrm{~d}, J=3.4 \mathrm{~Hz}, 1 \mathrm{H}), 4.42(\mathrm{q}, J=6.9 \mathrm{~Hz}, 1 \mathrm{H}), 1.71(\mathrm{~d}, J=6.9 \mathrm{~Hz}, 3 \mathrm{H}) ;{ }^{13} \mathrm{C}$ NMR $(100$ $\left.\mathrm{MHz}, \mathrm{D}_{2} \mathrm{O}\right): \delta 167.5,140.4,123.1,47.3,18.8$. IR (ATR): $v_{\max } 3282,2975,1673,1644,1495,1294$, 1189, 1049, 731, $642 \mathrm{~cm}^{-1}$; ESIHRMS m/z calcd for $\mathrm{C}_{5} \mathrm{H}_{8} \mathrm{~N}_{2} \mathrm{~S}[\mathrm{M}]^{+} 129.0481$, found 129.0483 . 


\section{Experimental Procedures and Characterization Data: Total Synthesis of Conulothiazole A}

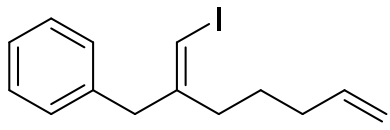

$11_{\mathrm{i}}$

\section{(E)-1-lodo-2-benzylhepta-1,6-diene $11_{\mathbf{i}}$.}

A three-necked round bottom flask equipped with a reflux condenser and an addition funnel was charged with magnesium turnings $(1.3 \mathrm{~g}, 53.5 \mathrm{mmol})$, placed under vacuum and backfilled with argon and the addition funnel was charged with dry diethyl ether ( $35 \mathrm{~mL}$ ) and 5-bromopent-1-ene (3.3 $\mathrm{mL}, 27.8 \mathrm{mmol})$. After having covered the magnesium turnings with diethyl ether, few drops of 5-bromopent-1-ene were added and the resulting mixture was gently stirred until the diethyl ether began to be disturbed. The solution of 5-bromopent-1-ene in diethyl ether was then added dropwise and the solution was stirred overnight at room temperature, yielding the desired solution of (pent-4-enyl)magnesium bromide which was finally titrated with 2-propanol and 1,10phenanthroline..$^{\mathrm{S}}$

Copper bromide $(3.0 \mathrm{~g}, 20.9 \mathrm{mmol})$ was charged in a $100 \mathrm{~mL}$ round bottom flask, washed with toluene, concentrated and dried under high vacuum for $30 \mathrm{~min}$. The flask was then evacuated under high vacuum, backfilled with argon and fitted with a rubber septum. Copper bromide was suspended in diethyl ether $(10.6 \mathrm{~mL})$ and cooled to $-40{ }^{\circ} \mathrm{C}$ before adding the freshly prepared solution of (pent-4-enyl)magnesium bromide (0.70 M in diethyl ether, $27 \mathrm{~mL}, 19.0 \mathrm{mmol})$. The resulting yellow homogeneous reaction mixture was stirred for $1 \mathrm{~h}$ at $-40{ }^{\circ} \mathrm{C}$ before adding 3-phenyl-1-propyne $10(2.2 \mathrm{~mL}, 17.7 \mathrm{mmol})$. The reaction mixture was allowed to warm slowly to $-20{ }^{\circ} \mathrm{C}$, stirred for $90 \mathrm{~min}$ then cooled to $-78{ }^{\circ} \mathrm{C}$ before adding a solution of iodine $(5.2 \mathrm{~g}, 20.5$

\footnotetext{
s8 The concentration of the solution of (pent-4-enyl)magnesium bromide was determined by using the following procedure: the solution of (pent-4-enyl)magnesium bromide $(5 \mathrm{~mL})$ was added to a solution of 1,10-phenanthroline $(5 \mathrm{mg})$ in diethyl ether $(5 \mathrm{~mL})$ under argon and the resulting light violet solution was cooled to $-78{ }^{\circ} \mathrm{C}$ and titrated with 2-propanol (slow addition until discoloration of the solution). A concentration of 0.70M was typically obtained.
} 
$\mathrm{mmol}$ ) in THF $(40 \mathrm{~mL})$. The reaction mixture was then allowed to warm slowly to room temperature, stirred overnight and then quenched with a mixture of pentane/3M aqueous solution of $\mathrm{HCl}(1 / 1 \mathrm{v} / \mathrm{v})$. The reaction mixture was filtered on a plug of Celite ${ }^{\circledR}$ and the organic layer was finally successively washed with a saturated aqueous solution of sodium bicarbonate $(100 \mathrm{~mL})$, a saturated aqueous solution of sodium thiosulfate $(100 \mathrm{~mL})$ and brine $(100 \mathrm{~mL})$, dried over anhydrous magnesium sulfate, filtered and concentrated. The crude residue was finally purified by flash column chromatography (petroleum ether) yielding the desired alkenyl iodide $\mathbf{1 1}_{\mathbf{i}}$ as a colorless oil (3.2 g, $10.3 \mathrm{mmol}, 60 \%) .{ }^{1} \mathrm{H}$ NMR (300 MHz, CDCl 3 ): $\delta$ 7.38-7.12 (m, 5H), $5.92(\mathrm{~s}$, 1H), 5.90-5.74 (m, 1H), $4.99(\mathrm{t}, J=18 \mathrm{~Hz}, 2 \mathrm{H}), 3.49(\mathrm{~s}, 2 \mathrm{H}), 2.17(\mathrm{t}, J=7.7 \mathrm{~Hz}, 2 \mathrm{H}), 2.07(\mathrm{q}, J=7.3$ $\mathrm{Hz}, 2 \mathrm{H}), 1.59-1.39(\mathrm{~m}, 2 \mathrm{H}) ;{ }^{13} \mathrm{C}$ NMR $\left(75 \mathrm{MHz}, \mathrm{CDCl}_{3}\right): \delta$ 150.7, 138.5, 138.3, 129.1 (2 C), 128.6 (2 C), 126.7, 115.0, 77.4, 43.6, 36.5, 33.6, 26.5; IR (ATR): $V_{\max } 2933,1640,1494,1543,912,735$, $699 \mathrm{~cm}^{-1}$; EIHRMS $\mathrm{m} / z$ calcd for $\mathrm{C}_{14} \mathrm{H}_{17} \mathrm{I}[\mathrm{M}]^{+} 312.0369$, found 312.0371 .

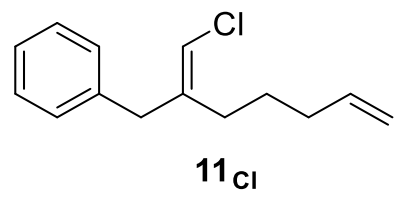

(E)-1-Chloro-2-benzylhepta-1,6-diene $11_{\mathrm{Cl}}$.

An oven-dried $5 \mathrm{~mL}$ resealable pressure tube was charged with copper iodide (I) (20 mg, 0.10 $\mathrm{mmol})$, tetramethylammonium chloride $(110 \mathrm{mg}, 1.00 \mathrm{mmol})$ and $(E)$-1-iodo-2-benzylhepta-1,6diene $\mathbf{1 1}_{\mathbf{i}}$ (156 $\mathrm{mg}, 0.50 \mathrm{mmol}$ ). The tube was fitted with a rubber septum, evacuated under vacuum, backfilled with argon before adding ethanol $(1 \mathrm{~mL})$ and trans- $N, N^{\prime}$-dimethylcyclohexane1,2-diamine ( $32 \mu \mathrm{L}, 0.20 \mathrm{mmol})$. The rubber septum was replaced by a Teflon-coated screwcap and the resulting suspension was stirred and heated at $110{ }^{\circ} \mathrm{C}$ in a preheated oil bath for $72 \mathrm{~h}$. The crude reaction mixture was cooled to room temperature, diluted with ethyl acetate, filtered on a plug of silica gel and concentrated under reduced pressure. The crude residue was finally purified by flash column chromatography over silica gel (petroleum ether) to afford the desired chlorinated alkene ${ }^{1} 1_{\mathrm{cl}}$ as a colorless oil (98 mg, $\left.0.44 \mathrm{mmol}, 89 \%\right) .{ }^{1} \mathrm{H} \mathrm{NMR}\left(300 \mathrm{MHz}, \mathrm{CDCl}_{3}\right): \delta$ 7.34-7.13 (m, 5H), $5.84(\mathrm{~s}, 1 \mathrm{H}), 5.87-5.72(\mathrm{~m}, 1 \mathrm{H}), 4.97(\mathrm{t}, J=18 \mathrm{~Hz}, 2 \mathrm{H}), 3.37(\mathrm{~s}, 2 \mathrm{H}), 2.17$ 
$(\mathrm{t}, J=7.7 \mathrm{~Hz}, 2 \mathrm{H}), 2.05(\mathrm{q}, J=7.3 \mathrm{~Hz}, 2 \mathrm{H}), 1.57-1.44(\mathrm{~m}, 2 \mathrm{H}) ;{ }^{13} \mathrm{C} \mathrm{NMR}\left(75 \mathrm{MHz}, \mathrm{CDCl}_{3}\right): \delta 142.0$, 138.5, 138.4, 129.0 (2 C), 128.6 (2 C), 126.6, 114.8, 114.3, 41.2, 33.6, 29.7, 26.3. IR (ATR): $v_{\max }$ 2927, 1640, 1494, 1453, 992, 911, 735, $699 \mathrm{~cm}^{-1}$; ElHRMS m/z calcd for $\mathrm{C}_{14} \mathrm{H}_{17} \mathrm{Cl}[\mathrm{M}]^{+} 220.1013$, found 220.1010 .

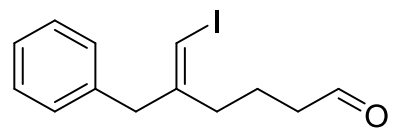

\section{(E)-5-Benzyl-6-iodohex-5-enal.}

$\mathrm{N}$-Methylmorpholine $\mathrm{N}$-oxide $(745 \mathrm{mg}, 6.36 \mathrm{mmol}$ ) and a solution of osmium tetroxide in water (4 wt.\% in water, $390 \mu \mathrm{L}, 58 \mu \mathrm{mol}$ ) were added to a solution of (E)-1-iodo-2-benzylhepta-1,6-diene $\mathbf{1 1}_{\mathbf{i}}(1.8 \mathrm{~g}, 5.8 \mathrm{mmol})$ in a tertiary mixture of tert-BuOH/THF/water $(5 / 5 / 1 \mathrm{v} / \mathrm{v} / \mathrm{v}, 56 \mathrm{~mL})$ at $0{ }^{\circ} \mathrm{C}$. The resulting reaction mixture was stirred for $18 \mathrm{~h}$ at room temperature then quenched with a saturated aqueous solution of sodium thiosulfate $(35 \mathrm{~mL})$ and stirred for an additional $1 \mathrm{~h}$. The aqueous layer was extracted thrice with diethyl ether $(70 \mathrm{~mL})$ and the combined organic layers were washed with brine $(70 \mathrm{~mL})$, dried over magnesium sulfate, filtered and concentrated under reduced pressure. The crude residue was used in the next step without further purification. Sodium periodate $(7.4 \mathrm{~g}, 34.6 \mathrm{mmol})$ was added to a solution of the intermediate diol in a binary mixture of $\mathrm{MeOH} /$ water $(2 / 1 \mathrm{v} / \mathrm{v}, 100 \mathrm{~mL})$ at room temperature. The resulting reaction mixture was stirred for $2 \mathrm{~h}$ and quenched with water $(35 \mathrm{~mL})$. The aqueous layer was extracted thrice with dichloromethane $(60 \mathrm{~mL})$ and the combined organic layers were washed with brine $(60 \mathrm{~mL})$, dried over magnesium sulfate, filtered and concentrated under reduced pressure to give the desired (E)-5-benzyl-6-iodohex-5-enal as an orange oil (1.4 g, $4.5 \mathrm{mmol}, 77 \%)$ which was used in the next step without further purification. ${ }^{1} \mathrm{H} \mathrm{NMR}\left(300 \mathrm{MHz}, \mathrm{CDCl}_{3}\right): \delta 9.77(\mathrm{t}, J=1.5 \mathrm{~Hz}, 1 \mathrm{H}), 7.36-7.11$ $(\mathrm{m}, 5 \mathrm{H}), 6.00(\mathrm{~s}, 1 \mathrm{H}), 3.50(\mathrm{~s}, 2 \mathrm{H}), 2.46(\mathrm{td}, J=1.5$ and $7.3 \mathrm{~Hz}, 2 \mathrm{H}), 2.25-2.15(\mathrm{~m}, 2 \mathrm{H}), 1.76$ (quint. $J=7.8 \mathrm{~Hz}, 2 \mathrm{H}$ ); ${ }^{13} \mathrm{C} \mathrm{NMR}\left(75 \mathrm{MHz}, \mathrm{CDCl}_{3}\right): \delta 202.2,149.9,138.0,129.0$ (2 C), 128.7 (2 C), 126.8, 78.4, 43.5, 43.2, 36.0, 19.6; IR (ATR): $v_{\max }$ 2940, 1722, 1494, 1453, 1259, 1075, 740, $700 \mathrm{~cm}^{1}$. 


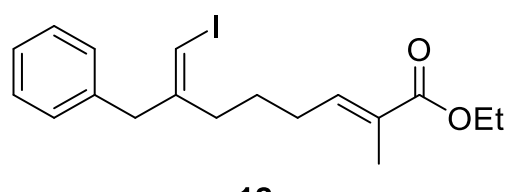

$12_{\mathrm{i}}$

\section{Ethyl (2E,7E)-7-benzyl-8-iodo-2-methylocta-2,7-dienoate $1 \mathbf{2}_{\text {i }}$.}

(Carbethoxyethylidene)triphenylphosphorane $(461 \mathrm{mg}, 1.27 \mathrm{mmol}$ ) was added to a solution of (E)-5-benzyl-6-iodohex-5-enal (200 $\mathrm{mg}, 0.64 \mathrm{mmol})$ in dichloromethane $(3 \mathrm{~mL})$ at $0{ }^{\circ} \mathrm{C}$ and stirred for $3 \mathrm{~h}$ at room temperature. The resulting reaction mixture was then concentrated under reduced pressure and the crude residue was finally purified by flash column chromatography over silica gel (petroleum ether/EtOAc: $95 / 5$ ) to yield the desired ester $\mathbf{1 2}_{\mathbf{i}}$ as a colorless oil $(231 \mathrm{mg}, 0.58 \mathrm{mmol}$, $91 \%) .{ }^{1} \mathrm{H}$ NMR $\left(300 \mathrm{MHz}, \mathrm{CDCl}_{3}\right): \delta 7.35-7.10(\mathrm{~m}, 5 \mathrm{H}), 6.75(\mathrm{t}, J=7.3 \mathrm{~Hz}, 1 \mathrm{H}), 5.96(\mathrm{~s}, 1 \mathrm{H}), 4.19$ (q, $J=7.1 \mathrm{~Hz}, 2 \mathrm{H}), 3.49(\mathrm{~s}, 2 \mathrm{H}), 2.24-2.13(\mathrm{~m}, 4 \mathrm{H}), 1.82(\mathrm{~s}, 3 \mathrm{H}), 1.61-1.48(\mathrm{~m}, 2 \mathrm{H}), 1.30(\mathrm{t}, J=7.1 \mathrm{~Hz}$, $3 \mathrm{H}) ;{ }^{13} \mathrm{C} \mathrm{NMR}\left(75 \mathrm{MHz}, \mathrm{CDCl}_{3}\right): \delta 168.2,150.2,141.5,138.1,129.0$ (2 C), 128.7 (2 C), 128.4, 126.7, 77.8, 60.5, 43.6, 36.6, 28.4, 26.2, 14.5, 12.6. IR (ATR): $v_{\max } 2942,1707,1695,1263,1187,1102$, 744, $700 \mathrm{~cm}^{-1}$; EIHRMS $\mathrm{m} / z$ calcd for $\mathrm{C}_{18} \mathrm{H}_{23} \mathrm{IO}_{2}[\mathrm{M}]^{+} 398.0737$, found 398.0740 .

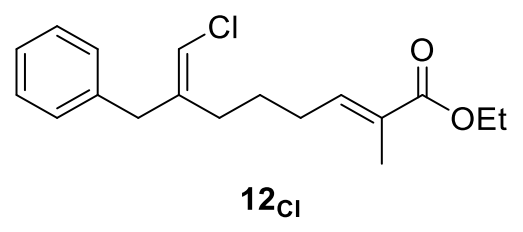

\section{Ethyl (2E,7E)-7-benzyl-8-chloro-2-methylocta-2,7-dienoate $12_{\mathrm{cl}}$.}

An oven-dried $15 \mathrm{~mL}$ resealable pressure tube was charged with copper iodide (I) (25 mg, 0.13 $\mathrm{mmol})$, tetramethylammonium chloride (146 mg, $1.33 \mathrm{mmol}$ ) and ethyl (2E,7E)-7-benzyl-8-iodo2-methylocta-2,7-dienoate $1 \mathbf{1 2}_{\mathbf{i}}(266 \mathrm{mg}, 0.67 \mathrm{mmol})$. The tube was fitted with a rubber septum, evacuated under vacuum, backfilled with argon before adding ethanol $(1.3 \mathrm{~mL})$ and trans- $N, N^{\prime}-$ dimethylcyclohexane-1,2-diamine $(42 \mu \mathrm{L}, 0.26 \mathrm{mmol})$. The rubber septum was replaced by a Teflon-coated screwcap and the resulting suspension was stirred and heated at $110{ }^{\circ} \mathrm{C}$ in a preheated oil bath for $72 \mathrm{~h}$. The crude reaction mixture was cooled to room temperature, diluted with ethyl acetate, filtered on a plug of silica gel and concentrated under reduced pressure. The 
crude residue was finally purified by flash column chromatography over silica gel (cyclohexane/EtOAc: 95/5) to afford the desired chlorinated alkene $\mathbf{1 2}_{\mathrm{cl}}$ as a colorless oil (182 $\mathrm{mg}$, $0.59 \mathrm{mmol}, 89$ \%). ${ }^{1} \mathrm{H}$ NMR $\left(300 \mathrm{MHz}, \mathrm{CDCl}_{3}\right): \delta 7.34-7.12(\mathrm{~m}, 5 \mathrm{H}), 6.73(\mathrm{t}, J=7.4 \mathrm{~Hz}, 1 \mathrm{H}), 5.87(\mathrm{~s}$, $1 \mathrm{H}), 4.19(\mathrm{q}, J=7.2 \mathrm{~Hz}, 2 \mathrm{H}), 3.37(\mathrm{~s}, 2 \mathrm{H}), 2.22-2.10(\mathrm{~m}, 4 \mathrm{H}), 1.81(\mathrm{~s}, 3 \mathrm{H}), 1.60-1.47(\mathrm{~m}, 2 \mathrm{H}), 1.30(\mathrm{t}$, $J=7.1 \mathrm{~Hz}, 3 \mathrm{H}) ;{ }^{13} \mathrm{C} \mathrm{NMR}\left(75 \mathrm{MHz}, \mathrm{CDCl}_{3}\right): \delta 168.3,141.6,138.2,129.1$ (2 C), 128.7 (2 C), 128.3, 126.7, 114.7, 60.6, 41.3, 29.9, 28.5, 26.1, 14.5, 12.5. IR (ATR): $v_{\max } 2931,1708,1454,1258,1109$, 1081, 733, $700 \mathrm{~cm}^{-1}$; ElHRMS $\mathrm{m} / z$ calcd for $\mathrm{C}_{18} \mathrm{H}_{23} \mathrm{ClO}_{2}[\mathrm{M}]^{+} 306.1381$, found 306.1383 .

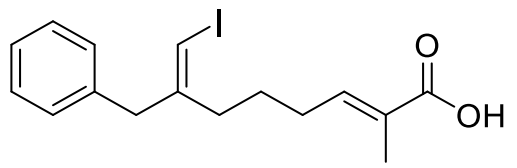

13 i

(2E,7E)-7-Benzyl-8-iodo-2-methylocta-2,7-dienoic acid 13i. A mixture of ethyl (2E,7E)-7-benzyl-8iodo-2-methylocta-2,7-dienoate 12 $(706 \mathrm{mg}, 1.77 \mathrm{mmol}$ ) and potassium hydroxide $(209 \mathrm{mg}, 3.72$ $\mathrm{mmol})$ in a binary mixture of $\mathrm{MeOH} /$ water $(1 / 1 \mathrm{v} / \mathrm{v}, 16 \mathrm{~mL})$ was stirred for $5 \mathrm{~h}$ at $80^{\circ} \mathrm{C}$ then cooled to room temperature. The resulting reaction mixture was diluted with ethyl acetate $(20 \mathrm{~mL})$ and the aqueous layer was carefully acidified to $\mathrm{pH} 1$ and extracted thrice with ethyl acetate (15 $\mathrm{mL})$. The combined organic layers were dried over magnesium sulfate, filtered and concentrated under reduced pressure. The crude residue was finally purified by flash column chromatography over silica gel (cyclohexane/EtOAc: 70/30) to afford the desired acid 13i (600 mg, $1.62 \mathrm{mmol}, 91$ \%) as a colorless oil. ${ }^{1} \mathrm{H}$ NMR $\left(300 \mathrm{MHz}, \mathrm{CDCl}_{3}\right): \delta$ 7.37-7.10 (m, 5H), $6.92(\mathrm{t}, J=7.3 \mathrm{~Hz}, 1 \mathrm{H}), 5.98(\mathrm{~s}, 1 \mathrm{H})$, $3.49(\mathrm{~s}, 2 \mathrm{H}), 2.28-2.15(\mathrm{~m}, 4 \mathrm{H}), 1.84(\mathrm{~s}, 3 \mathrm{H}), 1.56$ (quint., $J=7.5 \mathrm{~Hz}, 2 \mathrm{H}) ;{ }^{13} \mathrm{C} \mathrm{NMR}\left(75 \mathrm{MHz}, \mathrm{CDCl}_{3}\right)$ : $\delta$ 173.4, 150.1, 144.6, 138.1, 129.0 (2 C), 128.7 (2 C), 127.6, 126.8, 78.0, 43.7, 36.6, 28.7, 26.1, 12.3; IR (ATR): $v_{\max } 2943,1683,1642,1287,933,739,699 \mathrm{~cm}^{-1}$. 


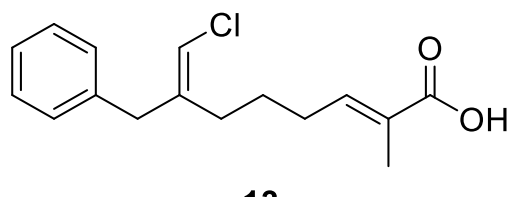

$13_{\mathrm{Cl}}$

(2E,7E)-7-Benzyl-8-chloro-2-methylocta-2,7-dienoic acid $13_{\mathrm{cl}}$. This compound was prepared according the two following procedures.

- A mixture of ethyl (2E,7E)-7-benzyl-8-chloro-2-methylocta-2,7-dienoate $12 \mathrm{cl}$ (476 mg, 1.55 $\mathrm{mmol}$ ) and potassium hydroxide $(183 \mathrm{mg}, 3.27 \mathrm{mmol})$ in a binary mixture of $\mathrm{MeOH} /$ water $(1 / 1$ $\mathrm{v} / \mathrm{v}, 15 \mathrm{~mL}$ ) was stirred for $5 \mathrm{~h}$ at $80^{\circ} \mathrm{C}$ then cooled to room temperature. The resulting reaction mixture was diluted with ethyl acetate $(50 \mathrm{~mL})$ and the aqueous layer was carefully acidified to $\mathrm{pH} 1$ and extracted thrice with ethyl acetate $(25 \mathrm{~mL})$. The combined organic layers were dried over magnesium sulfate, filtered and concentrated under reduced pressure. The crude residue was finally purified by flash column chromatography over silica gel (petroleum ether/EtOAc: $70 / 30$ ) to afford the desired acid $13_{\mathrm{cl}}$ as a pale yellow oil (425 $\mathrm{mg}, 1.52 \mathrm{mmol}, 98 \%$ ).

- An oven-dried $5 \mathrm{~mL}$ resealable pressure tube was charged with copper iodide (I) (3.5 mg, 18 $\mu \mathrm{mol})$, tetramethylammonium chloride $(19 \mathrm{mg}, 0.17 \mathrm{mmol})$ and $(2 E, 7 E)$-7-benzyl-8-iodo-2methylocta-2,7-dienoic acid 13i $(31 \mathrm{mg}, 84 \mu \mathrm{mol}$ ). The tube was fitted with a rubber septum, evacuated under vacuum, backfilled with argon before adding DMSO (170 $\mu \mathrm{L})$ and trans- $N, N^{\prime}-$ dimethylcyclohexane-1,2-diamine $(11 \mu \mathrm{L}, 70 \mu \mathrm{mol})$. The rubber septum was replaced by a Teflon-coated screwcap and the resulting suspension was stirred and heated at $110{ }^{\circ} \mathrm{C}$ in a preheated oil bath for $96 \mathrm{~h}$. The crude reaction mixture was cooled to rt, diluted with water $(15 \mathrm{~mL})$, extracted thrice with diethyl ether and the combined organic layers were dried over anhydrous magnesium sulfate, filtered and concentrated. The crude residue was finally purified by flash column chromatography over silica gel (petroleum ether/EtOAc: 70/30) to afford the desired acid $13_{\mathrm{cl}}$ as a pale yellow oil (14 $\left.\mathrm{mg}, 50 \mu \mathrm{mol}, 60 \%\right)$.

${ }^{1} \mathrm{H}$ NMR $\left(300 \mathrm{MHz}, \mathrm{CDCl}_{3}\right): \delta 11.88(\mathrm{~s}, 1 \mathrm{H}), 7.37-7.15(\mathrm{~m}, 5 \mathrm{H}), 6.93(\mathrm{t}, \mathrm{J}=7.3 \mathrm{~Hz}, 1 \mathrm{H}), 5.91(\mathrm{~s}$, $1 \mathrm{H}), 3.40(\mathrm{~s}, 2 \mathrm{H}), 2.29-2.16(\mathrm{~m}, 4 \mathrm{H}), 1.85(\mathrm{~s}, 3 \mathrm{H}), 1.58$ (quint., $J=8.0 \mathrm{~Hz}, 2 \mathrm{H}) ;{ }^{13} \mathrm{C} \mathrm{NMR}(75 \mathrm{MHz}$, $\mathrm{CDCl}_{3}$ ): $\delta$ 173.7, 144.7, 141.5, 138.2, 129.1 (2 C), 128.7 (2 C), 127.6, 126.8, 114.8, 41.3, 29.8, 28.7, 25.9, 12.2; IR (ATR): $v_{\max } 2928,1684,1641,1454,1288,909,782,734,700 \mathrm{~cm}^{-1}$. 


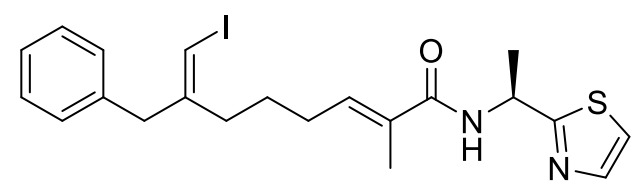

iodo-conulothiazole $A\left(\mathbf{1}_{\mathbf{i}}\right)$

Iodo-conulothiazole A $\mathbf{1}_{\mathbf{i}}$. To a solution of $(2 E, 7 E)$-7-benzyl-8-iodo-2-methylocta-2,7-dienoic acid 13i $(200 \mathrm{mg}, 0.54 \mathrm{mmol})$ in DMF $(7.0 \mathrm{~mL})$ under argon were successively added (S)-2-(1aminoethyl)thiazole 9 (144 mg, $1.10 \mathrm{mmol})$, 1-ethyl-3-(3-dimethylaminopropyl) carbodiimide hydrochloride (216 mg, $1.11 \mathrm{mmol})$, 1-hydroxybenzotriazole $(80 \mathrm{mg}, 0.59 \mathrm{mmol}$ ) and triethylamine $(157 \mu \mathrm{L}, 1.11 \mathrm{mmol})$. The resulting reaction mixture was stirred at room temperature for $12 \mathrm{~h}$ then diluted with ethyl acetate and washed successively with a saturated aqueous solution of ammonium chloride and brine. The organic layer was then dried over magnesium sulfate, filtered and concentrated under reduced pressure. The crude residue was finally purified by flash column chromatography over silica gel (petroleum ether/EtOAc: 50/50) to yield the desired iodo-conulothiazole $A \mathbf{1}_{\mathbf{i}}$ as a yellow oil (205 mg, $\left.0.42 \mathrm{mmol}, 79 \%\right)$. [ $\left.\alpha\right]_{\mathrm{D}}^{25}-4.8$ ( $c$ 8.3, $\left.\mathrm{CHCl}_{3}\right) ;{ }^{1} \mathrm{H}$ NMR (400 MHz, CD $\left.\mathrm{OD}\right): \delta 7.71(\mathrm{~d}, J=3.6 \mathrm{~Hz}, 1 \mathrm{H}), 7.47$ (d, J = 3.2 Hz, 1H), 7.30-7.24 (m, 2H), 7.24-7.15 (m, 3H), $6.35(\mathrm{td}, J=7.2 \mathrm{~Hz}$ and $1.2 \mathrm{~Hz}, 1 \mathrm{H}), 6.07(\mathrm{~s}, 1 \mathrm{H}), 5.40(\mathrm{q}, J=7.2 \mathrm{~Hz}, 1 \mathrm{H})$, $3.53(\mathrm{~s}, 2 \mathrm{H}), 2.25-2.13(\mathrm{~m}, 4 \mathrm{H}), 1.84(\mathrm{~d}, J=0.8 \mathrm{~Hz}, 3 \mathrm{H}), 1.62(\mathrm{~d}, J=7.2 \mathrm{~Hz}, 3 \mathrm{H}), 1.59-1.49(\mathrm{~m}, 2 \mathrm{H})$; ${ }^{13} \mathrm{C}$ NMR (100 MHz, CD $\left.{ }_{3} \mathrm{OD}\right): \delta 176.4,171.9,151.9,143.1,139.7,137.5,132.4,129.9,129.5,127.6$, 120.3, 77.7, 44.3, 37.3, 29.0, 27.3, 20.9, 12.9; IR (ATR): $v_{\max } 3303,2936,1660,1622,1496,1452$, $1187,1146,1052,732,700,641 \mathrm{~cm}^{-1}$; ESIHRMS $\mathrm{m} / z$ calcd for $\mathrm{C}_{21} \mathrm{H}_{26} \mathrm{IN}_{2} \mathrm{OS}[\mathrm{M}+\mathrm{H}]^{+} 481.0805$, found 481.0806 . 


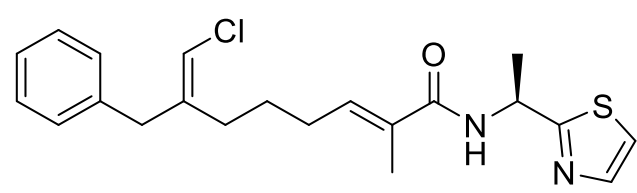

conulothiazole A (1)

Conulothiazole $\mathbf{A}(\mathbf{1})$. This compound was prepared according the two following procedures.

- To a solution of (2E,7E)-7-benzyl-8-chloro-2-methylocta-2,7-dienoic acid $13 \mathrm{cl}(120 \mathrm{mg}, 0.43$ $\mathrm{mmol}$ ) in DMF (6 mL) under argon were successively added (S)-2-(1-aminoethyl)thiazole 9 (115 $\mathrm{mg}, 0.90 \mathrm{mmol}$ ), 1-ethyl-3-(3-dimethylaminopropyl) carbodiimide hydrochloride (172 mg, 0.90 mmol), 1-hydroxybenzotriazole (64 mg, $0.47 \mathrm{mmol}$ ) and triethylamine (125 $\mu \mathrm{L}, 0.90 \mathrm{mmol}$ ). The resulting reaction mixture was stirred at room temperature for $24 \mathrm{~h}$ then diluted with ethyl acetate and washed successively with a saturated aqueous solution of ammonium chloride and brine. The organic layer was finally dried over magnesium sulfate, filtered and concentrated under reduced pressure. The crude residue was finally purified by flash column chromatography over silica gel (petroleum ether/EtOAc: 50/50) to yield the desired conulothiazole A 1 as a pale yellow oil (137 mg, $0.35 \mathrm{mmol}, 82 \%)$.

- An oven-dried $5 \mathrm{~mL}$ resealable pressure tube was charged with copper iodide (I) (6 mg, 32 $\mu \mathrm{mol})$, tetramethylammonium chloride $(70 \mathrm{mg}, 0.64 \mathrm{mmol})$ and iodo-conulothiazole A $\mathbf{1}_{\mathbf{i}}$ (77 $\mathrm{mg}, 0.16 \mathrm{mmol})$. The tube was fitted with a rubber septum, evacuated under vacuum, backfilled with argon before adding DMSO (320 $\mu \mathrm{L})$ and trans- $N, N^{\prime}$-dimethylcyclohexane-1,2diamine $(10 \mu \mathrm{L}, 64 \mu \mathrm{mol})$. The rubber septum was replaced by a Teflon-coated screwcap and the resulting suspension was stirred and heated at $110{ }^{\circ} \mathrm{C}$ in a preheated oil bath for $72 \mathrm{~h}$. The crude reaction mixture was cooled to $\mathrm{rt}$, diluted with water $(15 \mathrm{~mL})$, extracted thrice with diethyl ether and the combined organic layers were dried over anhydrous magnesium sulfate, filtered and concentrated. The crude residue was finally purified by flash column chromatography over silica gel (petroleum ether/EtOAc: 50/50) to afford the desired conulothiazole A 1 as a pale yellow oil (62 mg, $0.16 \mathrm{mmol}$, quant.).

$[\alpha]_{\mathrm{D}}^{25}-8.5\left(c\right.$ 8.33, $\left.\mathrm{CHCl}_{3}\right) ;{ }^{1} \mathrm{H} \mathrm{NMR}\left(600 \mathrm{MHz}, \mathrm{CD}_{3} \mathrm{OD}\right): \delta 7.71(\mathrm{~d}, J=3 \mathrm{~Hz}, 1 \mathrm{H}), 7.47(\mathrm{~d}, J=3 \mathrm{~Hz}$, $1 \mathrm{H}), 7.27(\mathrm{~d}, J=7.2 \mathrm{~Hz}, 2 \mathrm{H}), 7.22-7.11(\mathrm{~m}, 3 \mathrm{H}), 6.34(\mathrm{t}, J=7.2 \mathrm{~Hz}, 1 \mathrm{H}), 6.00(\mathrm{~s}, 1 \mathrm{H}), 5.40(\mathrm{q}, J=$ 
$6.8 \mathrm{~Hz}, 1 \mathrm{H}), 3.40(\mathrm{~s}, 2 \mathrm{H}), 2.20(\mathrm{t}, J=7.8 \mathrm{~Hz}, 2 \mathrm{H}), 2.16(\mathrm{q}, J=7.2 \mathrm{~Hz}, 2 \mathrm{H}), 1.82(\mathrm{~s}, 3 \mathrm{H}), 1.61(\mathrm{~d}, J=$ $7.2 \mathrm{~Hz}, 3 \mathrm{H}$ ), 1.53 (quint, $J=7.2 \mathrm{~Hz}, 2 \mathrm{H}) ;{ }^{13} \mathrm{C} \mathrm{NMR}\left(150 \mathrm{MHz}, \mathrm{CD}_{3} \mathrm{OD}\right): \delta 176.5,171.9,143.3$, 143.1, 139.7, 137.6, 132.3, 130.0 (2 C), 129.5 (2 C), 127.6, 120.3, 115.3, 48.8, 42.0, 30.5, 29.0, 27.1, 20.9, 12.9. IR (ATR): $v_{\max }$ 3292, 2933, 1661, 1627, 1516, 1453, 1148, 752, $701 \mathrm{~cm}^{-1}$; ESIHRMS $m / z$ calcd for $\mathrm{C}_{21} \mathrm{H}_{26} \mathrm{ClN}_{2} \mathrm{OS}[\mathrm{M}+\mathrm{H}]^{+} 389.1449$, found 389.1449. 


\section{Conulothiazole A Spectral Data Compared to Reported Data}

${ }^{1} \mathrm{H}$ NMR (Synthetic at $600 \mathrm{MHz}$; Natural at $700 \mathrm{MHz}$ )

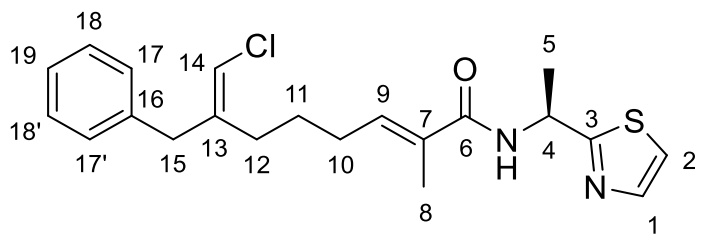

conulothiazole A (1)

\begin{tabular}{|c|c|c|c|c|c|}
\hline \multirow[b]{2}{*}{ Position } & \multicolumn{2}{|c|}{ Synthetic } & \multicolumn{2}{|c|}{ Natural ${ }^{\mathrm{S9}}$} & \multirow[b]{2}{*}{$\Delta \delta$} \\
\hline & $\delta$ & multiplicity & $\delta$ & multiplicity & \\
\hline $1(\mathrm{CH})$ & 7.71 & $\mathrm{~d}, J=3.0 \mathrm{~Hz}$ & 7.71 & $\mathrm{~d}, J=3.4 \mathrm{~Hz}$ & 0.00 \\
\hline $2(\mathrm{CH})$ & 7.47 & $\mathrm{~d}, J=3.0 \mathrm{~Hz}$ & 7.48 & $\mathrm{~d}, J=3.3 \mathrm{~Hz}$ & -0.01 \\
\hline $4(\mathrm{CH})$ & 5.40 & $q, J=6.6 \mathrm{~Hz}$ & 5.39 & $q, J=7.1 \mathrm{~Hz}$ & 0.01 \\
\hline $5\left(\mathrm{CH}_{3}\right)$ & 1.61 & $\mathrm{~d}, J=7.2 \mathrm{~Hz}$ & 1.63 & $\mathrm{~d}, J=7.1 \mathrm{~Hz}$ & -0.02 \\
\hline $8\left(\mathrm{CH}_{3}\right)$ & 1.82 & $\mathrm{~s}$ & 1.83 & $\mathrm{~s}$ & -0.01 \\
\hline $9(\mathrm{CH})$ & 6.34 & $\mathrm{t}, J=7.2 \mathrm{~Hz}$ & 6.34 & $\mathrm{tq}, J=7.4,1.4 \mathrm{~Hz}$ & 0.00 \\
\hline $10\left(\mathrm{CH}_{2}\right)$ & 2.16 & $\mathrm{q}, J=7.2 \mathrm{~Hz}$ & 2.16 & $\mathrm{q}, J=7.5 \mathrm{~Hz}$ & 0.00 \\
\hline $11\left(\mathrm{CH}_{2}\right)$ & 1.53 & quint, $J=7.2 \mathrm{~Hz}$ & 1.55 & quint, $J=7.7 \mathrm{~Hz}$ & -0.02 \\
\hline $12\left(\mathrm{CH}_{2}\right)$ & 2.20 & $\mathrm{t}, J=7.8 \mathrm{~Hz}$ & 2.20 & $\mathrm{t}, J=7.9 \mathrm{~Hz}$ & 0.00 \\
\hline $14(\mathrm{CH})$ & 6.00 & $\mathrm{~s}$ & 6.02 & $\mathrm{~s}$ & -0.02 \\
\hline $15\left(\mathrm{CH}_{2}\right)$ & 3.40 & $\mathrm{~s}$ & 3.42 & $\mathrm{~s}$ & -0.02 \\
\hline $17 / 17^{\prime}(\mathrm{CH})$ & 7.18 & $\mathrm{~d}, J=7.8 \mathrm{~Hz}$ & 7.19 & $\mathrm{~d}, J=7.8 \mathrm{~Hz}$ & -0.01 \\
\hline $18 / 18^{\prime}(\mathrm{CH})$ & 7.27 & $\mathrm{t}, \mathrm{J}=7.2 \mathrm{~Hz}$ & 7.28 & $\mathrm{t}, \mathrm{J}=7.8 \mathrm{~Hz}$ & -0.01 \\
\hline $19(\mathrm{CH})$ & $7.22-7.11$ & $\mathrm{~m}$ & 7.20 & $\mathrm{~d}, J=7.8 \mathrm{~Hz}$ & 0.00 \\
\hline
\end{tabular}

s9 Esposito, G.; Della Sala, G.; Teta, R.; Caso, A.; Bourguet-Kondracki, M.-L.; Pawlik, J. R.; Mangoni, A.; Costantino V. Eur. J. Org. Chem. 2016, 2871. 
${ }^{13} \mathrm{C}$ NMR (Synthetic at $150 \mathrm{MHz}$; Natural at $175 \mathrm{MHz}$ )

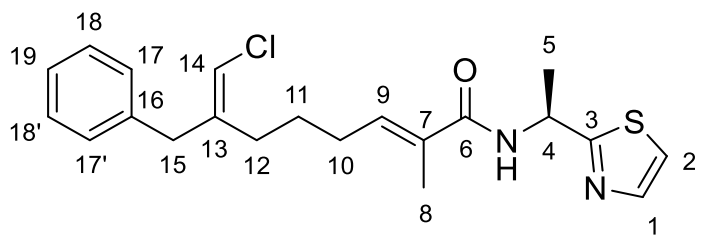

conulothiazole A (1)

\begin{tabular}{|c|c|c|c|}
\cline { 2 - 3 } \multicolumn{1}{c|}{} & Synthetic & Natural $^{\mathrm{S} 9}$ & \multicolumn{1}{c|}{} \\
\hline Position & $\delta$ & $\delta$ & $\Delta \delta$ \\
\hline $1(\mathrm{CH})$ & 143.1 & 142.9 & 0.2 \\
\hline $2(\mathrm{CH})$ & 120.3 & 120.5 & -0.2 \\
\hline $3(\mathrm{C})$ & 176.5 & 176.7 & -0.2 \\
\hline $4(\mathrm{CH})$ & 48.8 & 48.9 & -0.1 \\
\hline $5\left(\mathrm{CH}_{3}\right)$ & 20.9 & 20.9 & 0.0 \\
\hline $6(\mathrm{C})$ & 171.9 & 172.3 & -0.4 \\
\hline $7(\mathrm{C})$ & 132.3 & 132.5 & -0.2 \\
\hline $8\left(\mathrm{CH}_{3}\right)$ & 12.9 & 12.8 & 0.1 \\
\hline $9\left(\mathrm{CH}^{n}\right.$ & 137.6 & 137.7 & -0.1 \\
\hline $10\left(\mathrm{CH}_{2}\right)$ & 29.0 & 29.1 & -0.1 \\
\hline $11\left(\mathrm{CH}_{2}\right)$ & 27.1 & 27.2 & -0.1 \\
\hline $12\left(\mathrm{CH}_{2}\right)$ & 30.5 & 30.5 & 0.0 \\
\hline $13(\mathrm{C})$ & 143.3 & 143.5 & -0.2 \\
\hline $14(\mathrm{CH})$ & 115.3 & 115.4 & -0.1 \\
\hline $15(\mathrm{CH})_{2}$ & 42.0 & 42.0 & 0.1 \\
\hline $16(\mathrm{C})$ & 139.7 & 139.9 & -0.2 \\
\hline $17 / 21(\mathrm{CH})$ & 130.0 & 130.1 & -0.1 \\
\hline $18 / 20(\mathrm{CH})$ & 129.5 & 129.7 & -0.2 \\
\hline $19(\mathrm{CH})$ & 127.6 & 127.7 & -0.1 \\
\hline
\end{tabular}


Supporting Information

\section{${ }^{1} \mathrm{H}$ and ${ }^{13} \mathrm{C}$ NMR spectra}



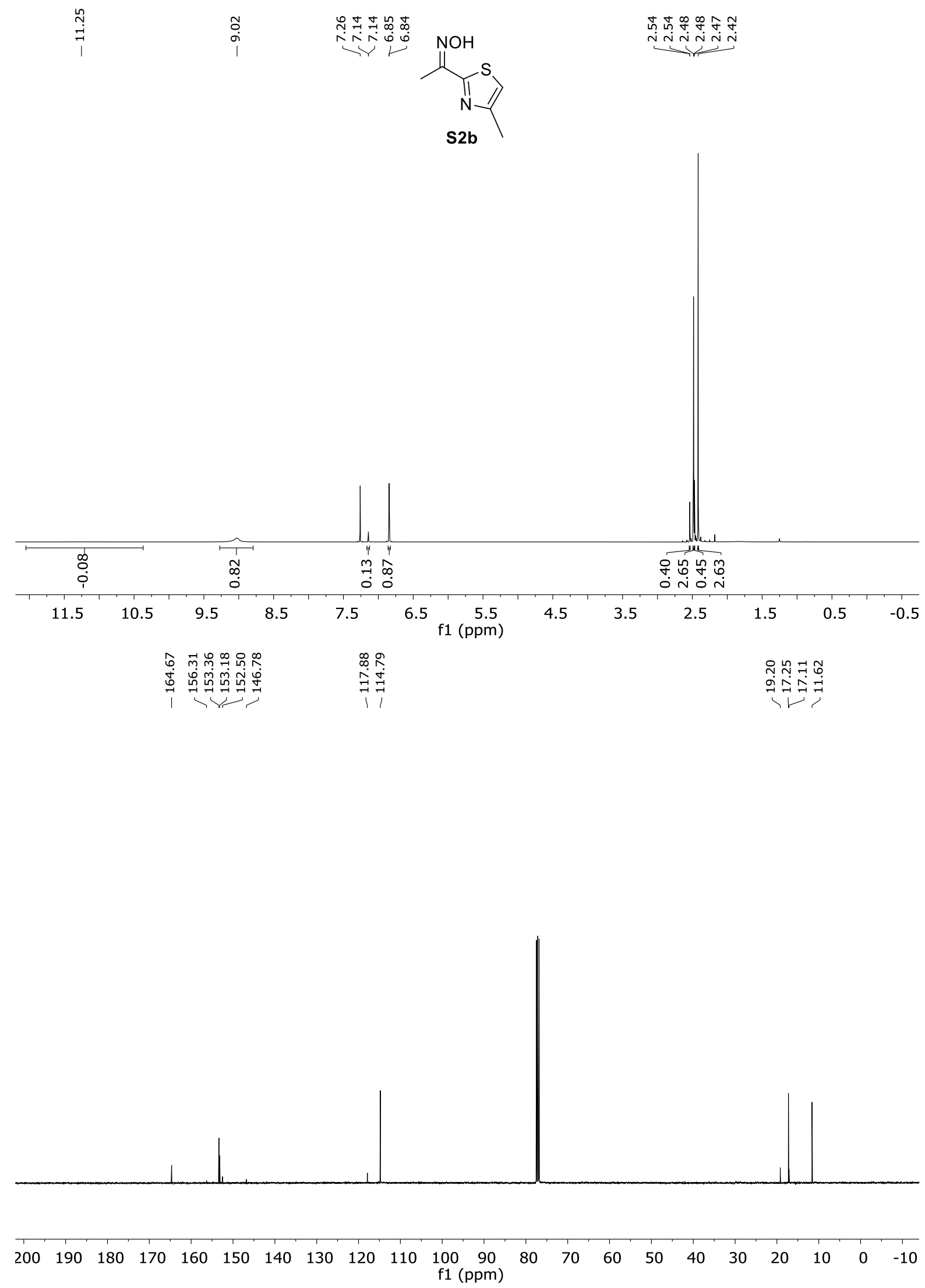

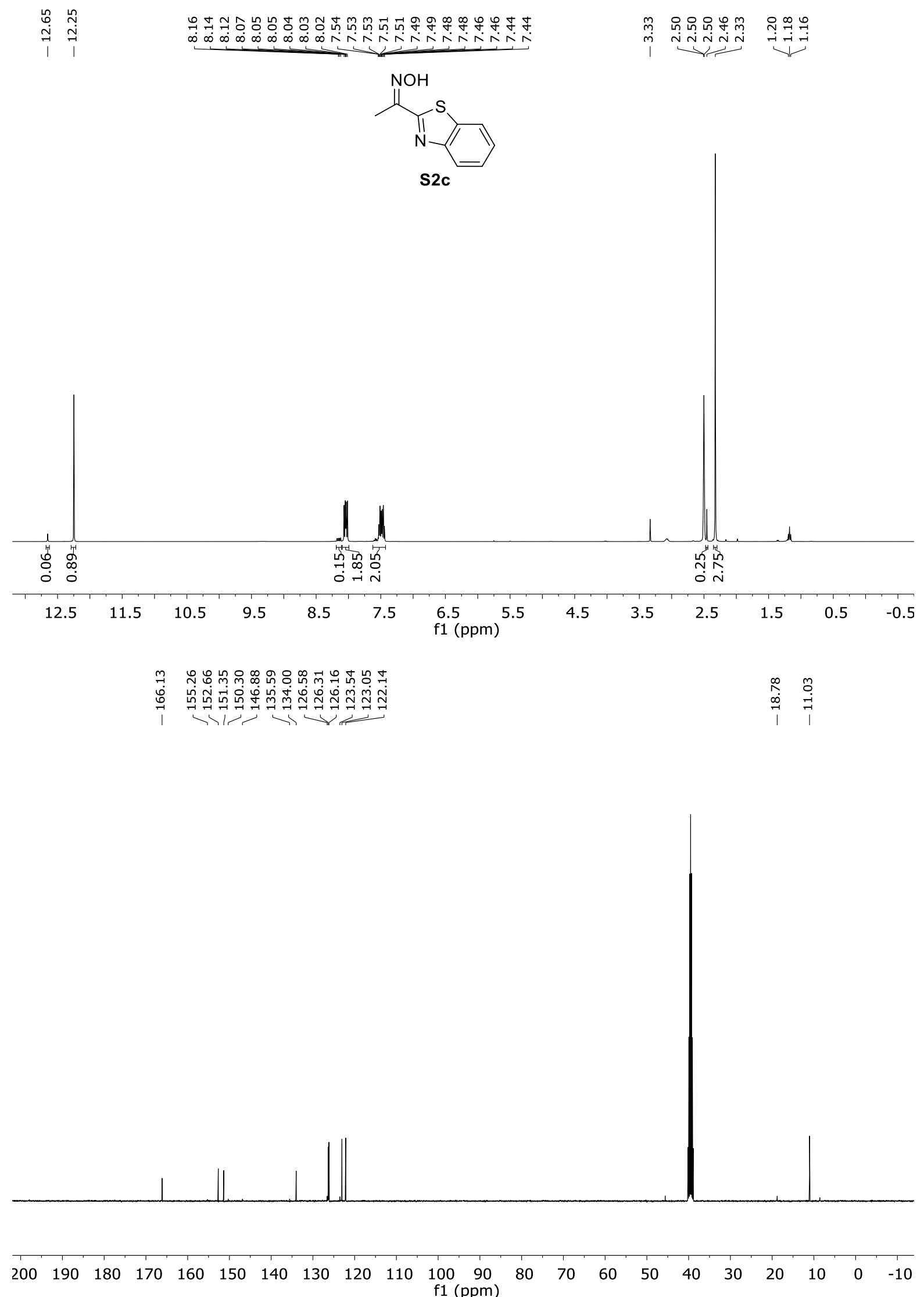


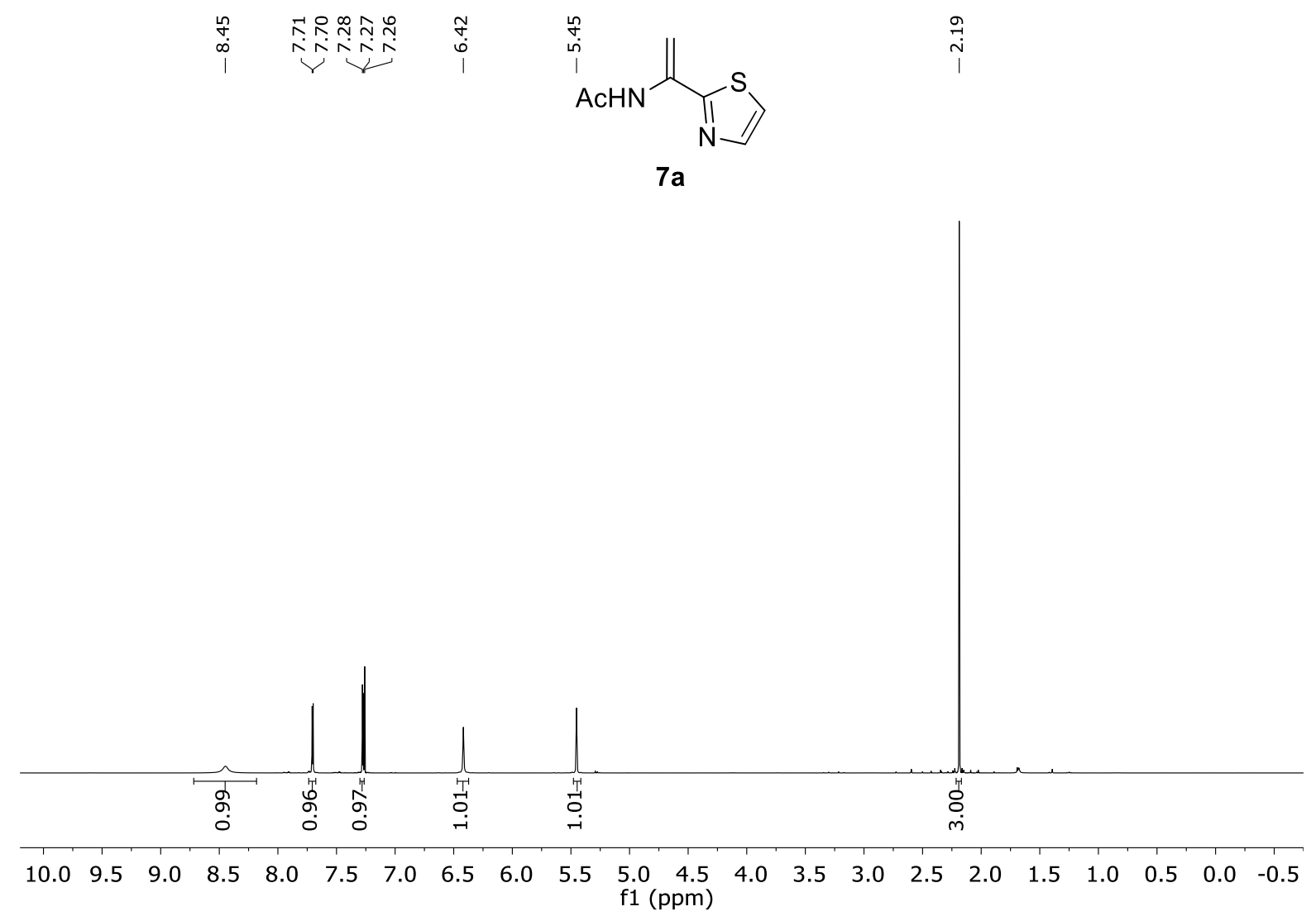

\begin{tabular}{|c|c|c|c|}
\hline 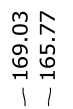 & $\begin{array}{l}\underset{\sim}{-1} \\
\stackrel{\sim}{*} \\
\stackrel{+}{1} \\
1\end{array}$ & $\begin{array}{l}\text { ने } \\
\stackrel{-}{m} \\
\stackrel{-}{\mid}\end{array}$ & $\begin{array}{l}\vec{\infty} \\
\infty \\
\stackrel{-1}{-1} \\
-1 \\
1\end{array}$ \\
\hline
\end{tabular}

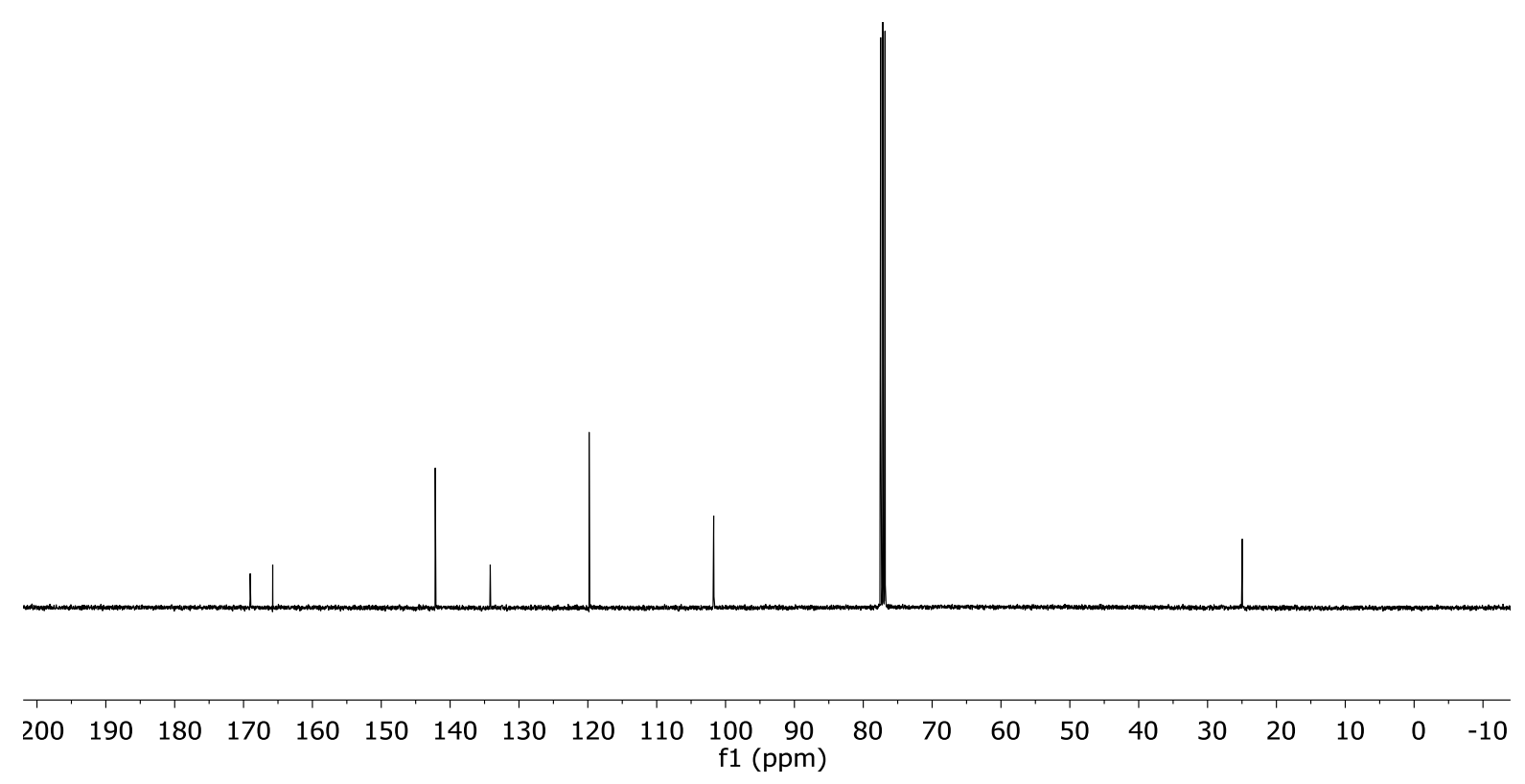



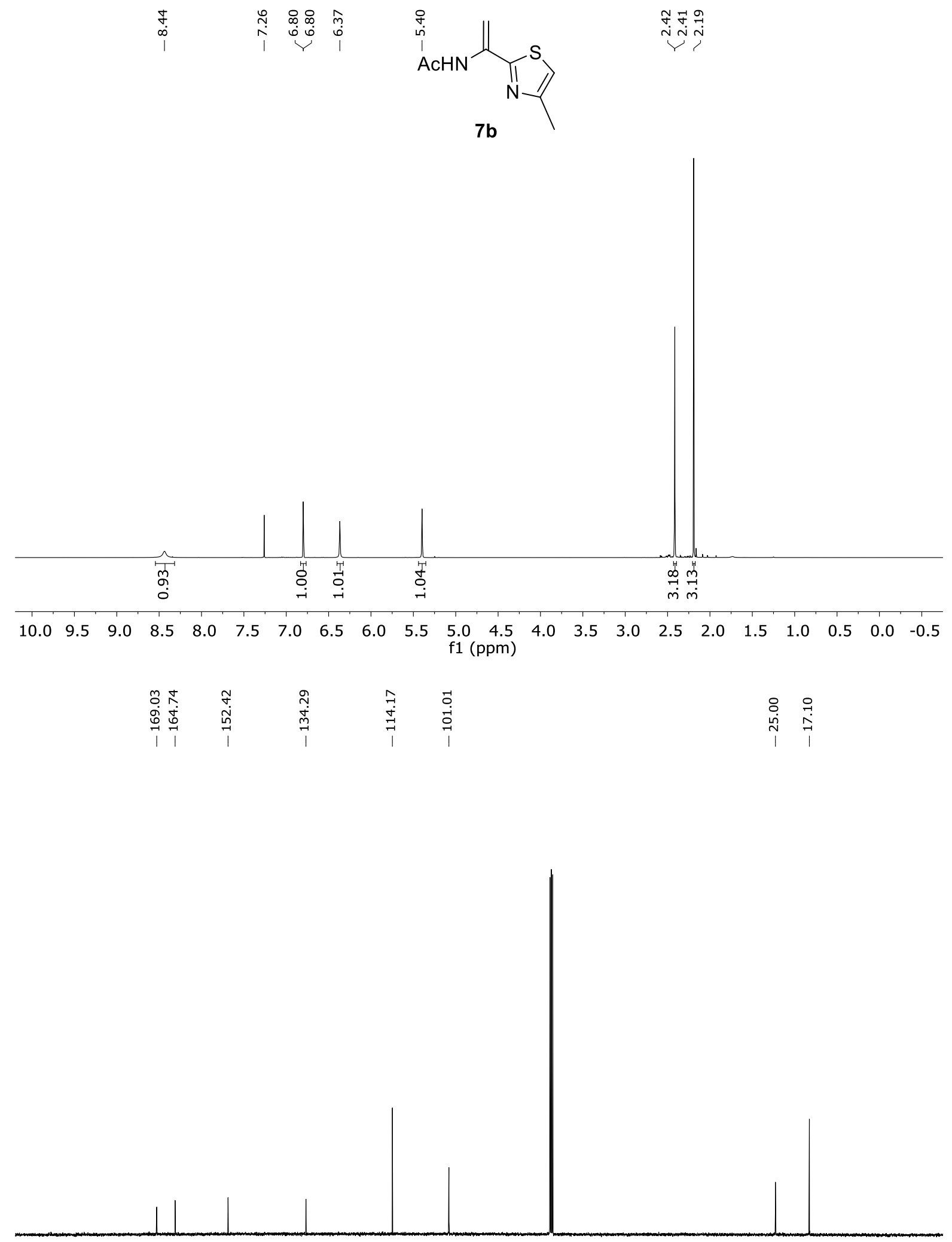

$\begin{array}{llllllllllllllllllllll}200 & 190 & 180 & 170 & 160 & 150 & 140 & 130 & 120 & 110 & \underset{f 10}{\mathrm{f} 1(\mathrm{ppm})} & 90 & 80 & 70 & 60 & 50 & 40 & 30 & 20 & 10 & 0 & -10\end{array}$ 


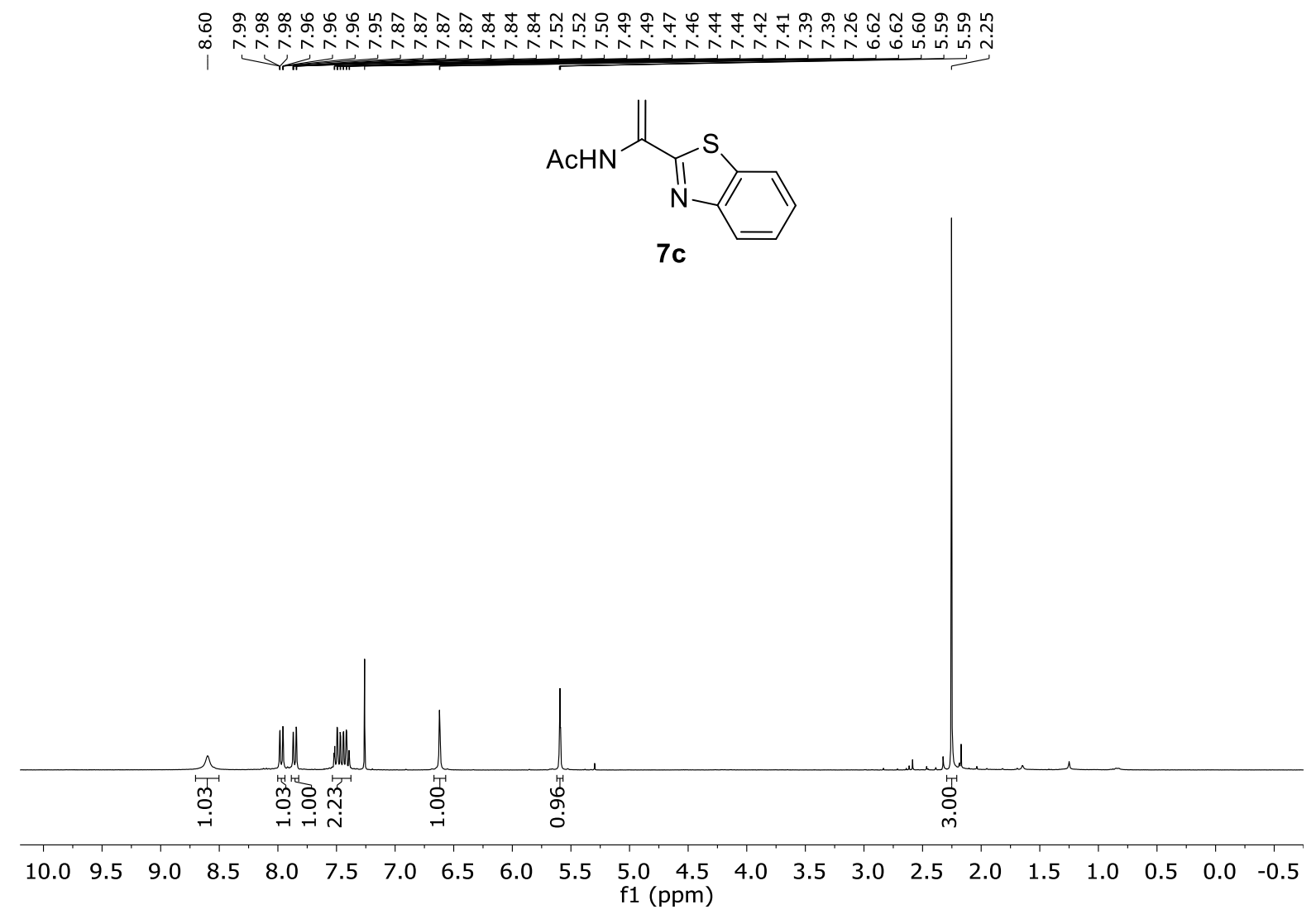

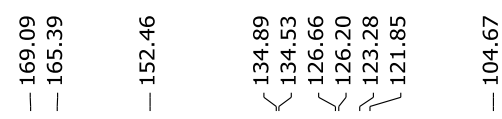

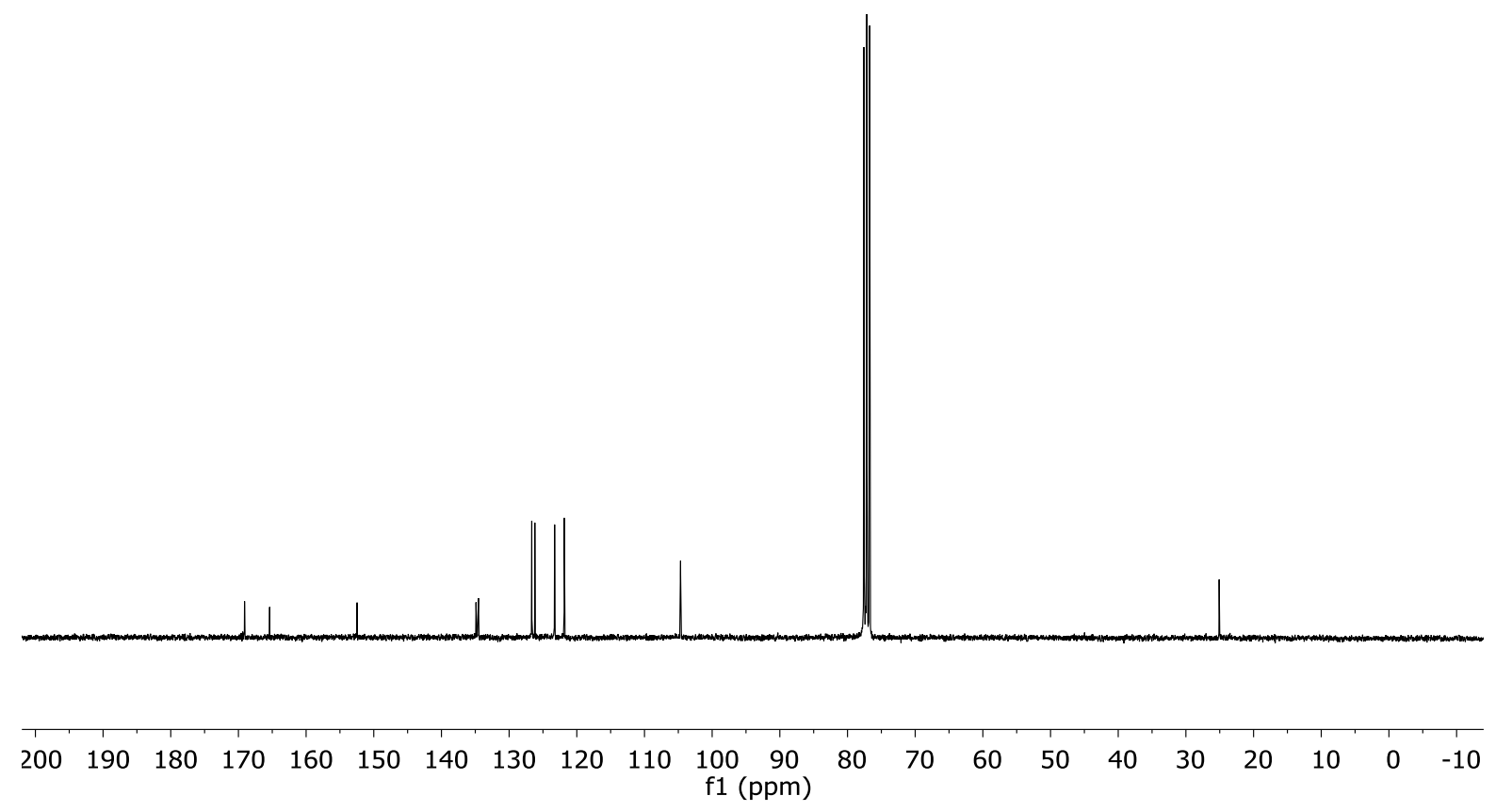



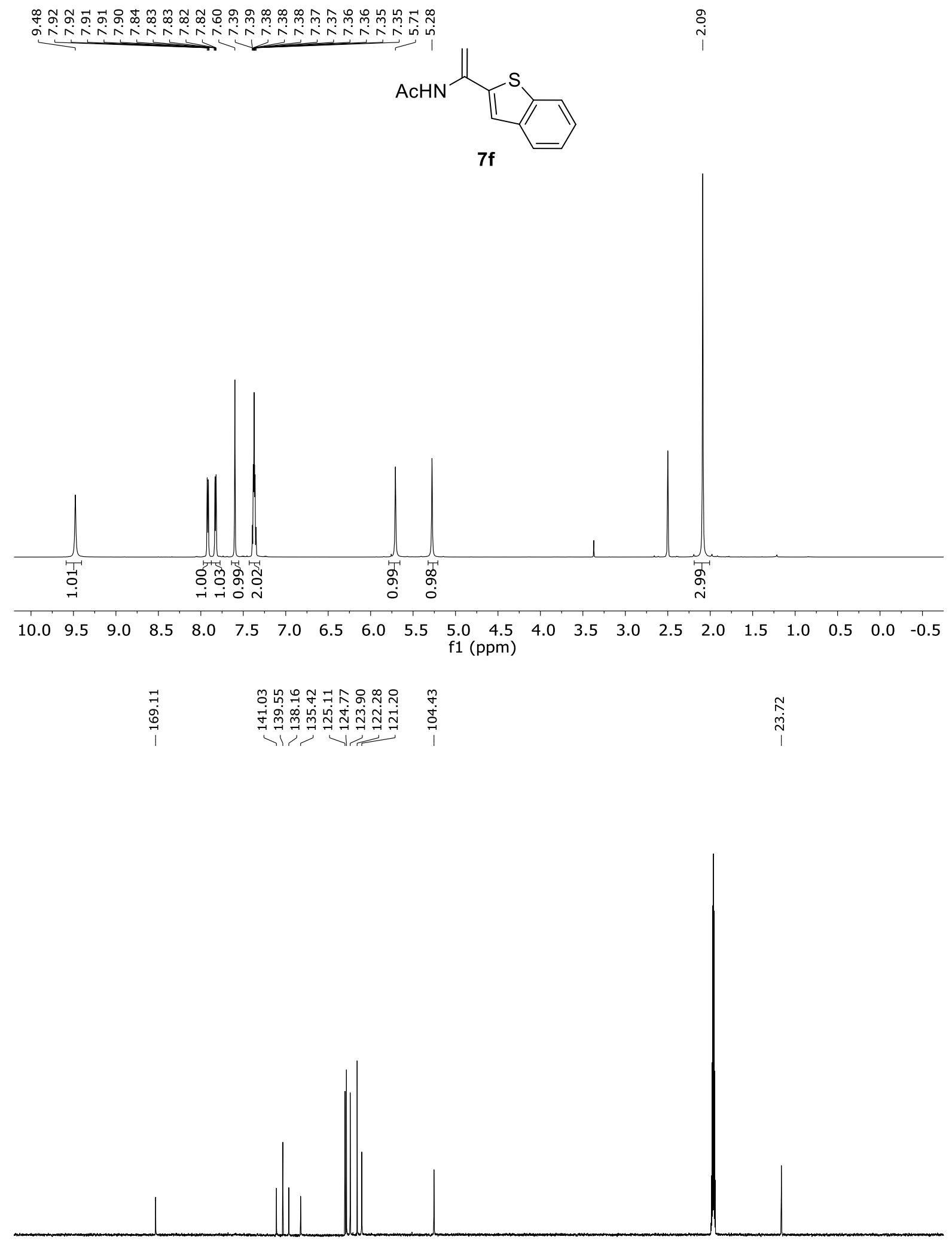

$\begin{array}{llllllllllllllllllllll}200 & 190 & 180 & 170 & 160 & 150 & 140 & 130 & 120 & 110 & \underset{f 1}{\mathrm{f} 1}(\mathrm{ppm}) & 90 & 80 & 70 & 60 & 50 & 40 & 30 & 20 & 10 & 0 & -10\end{array}$ 


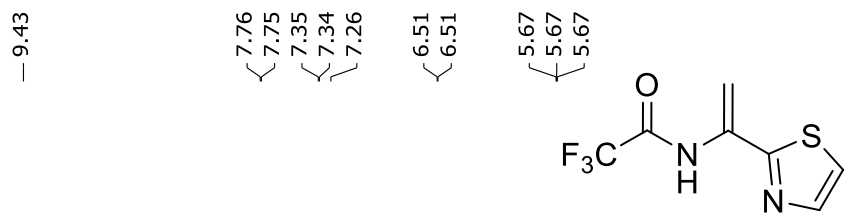

$7 \mathrm{~h}$
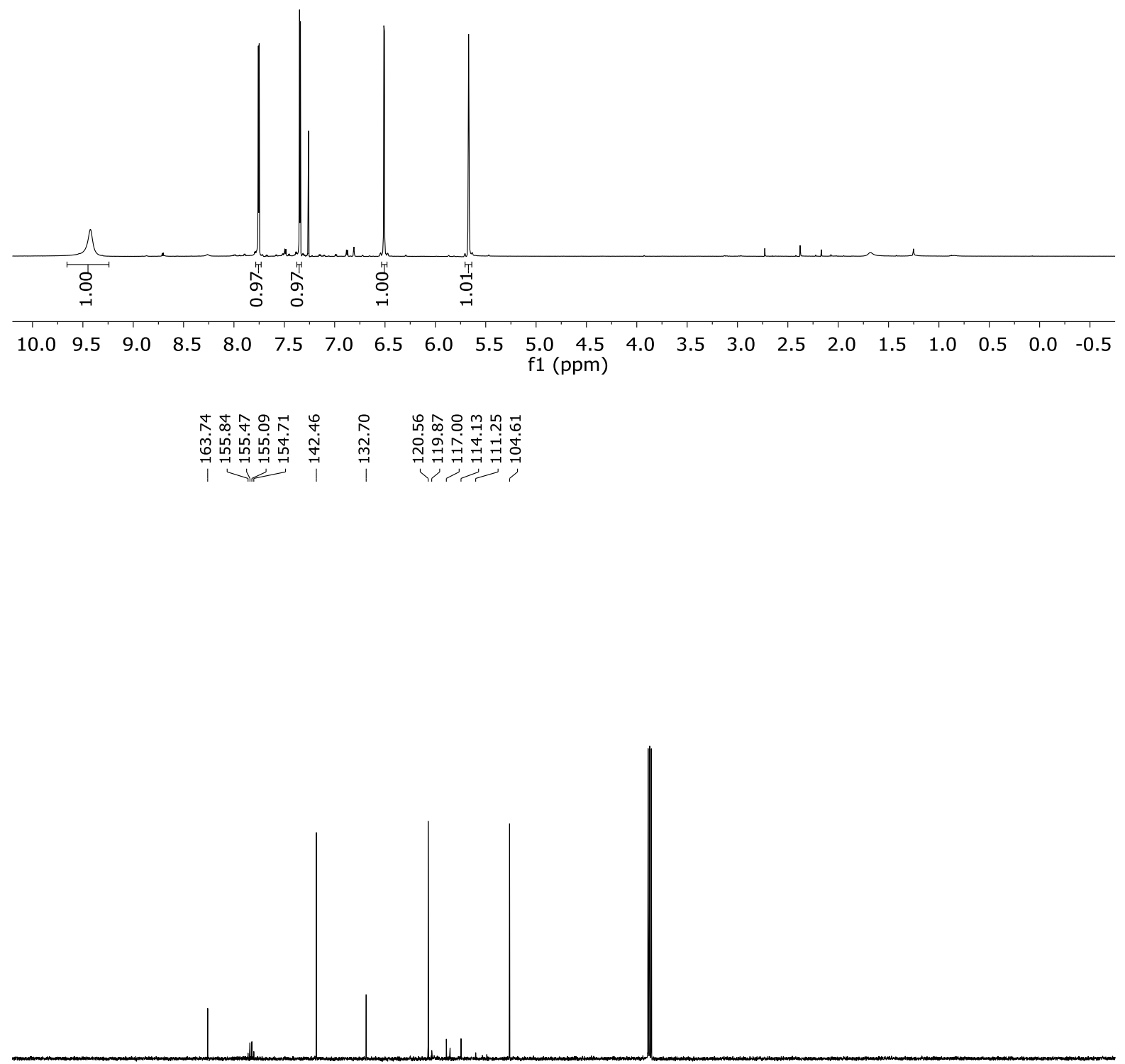

$\begin{array}{llllllllllllllllllllll}200 & 190 & 180 & 170 & 160 & 150 & 140 & 130 & 120 & 110 & \begin{array}{c}100 \\ \mathrm{f} 1(\mathrm{ppm})\end{array} & 80 & 70 & 60 & 50 & 40 & 30 & 20 & 10 & 0 & -10\end{array}$ 

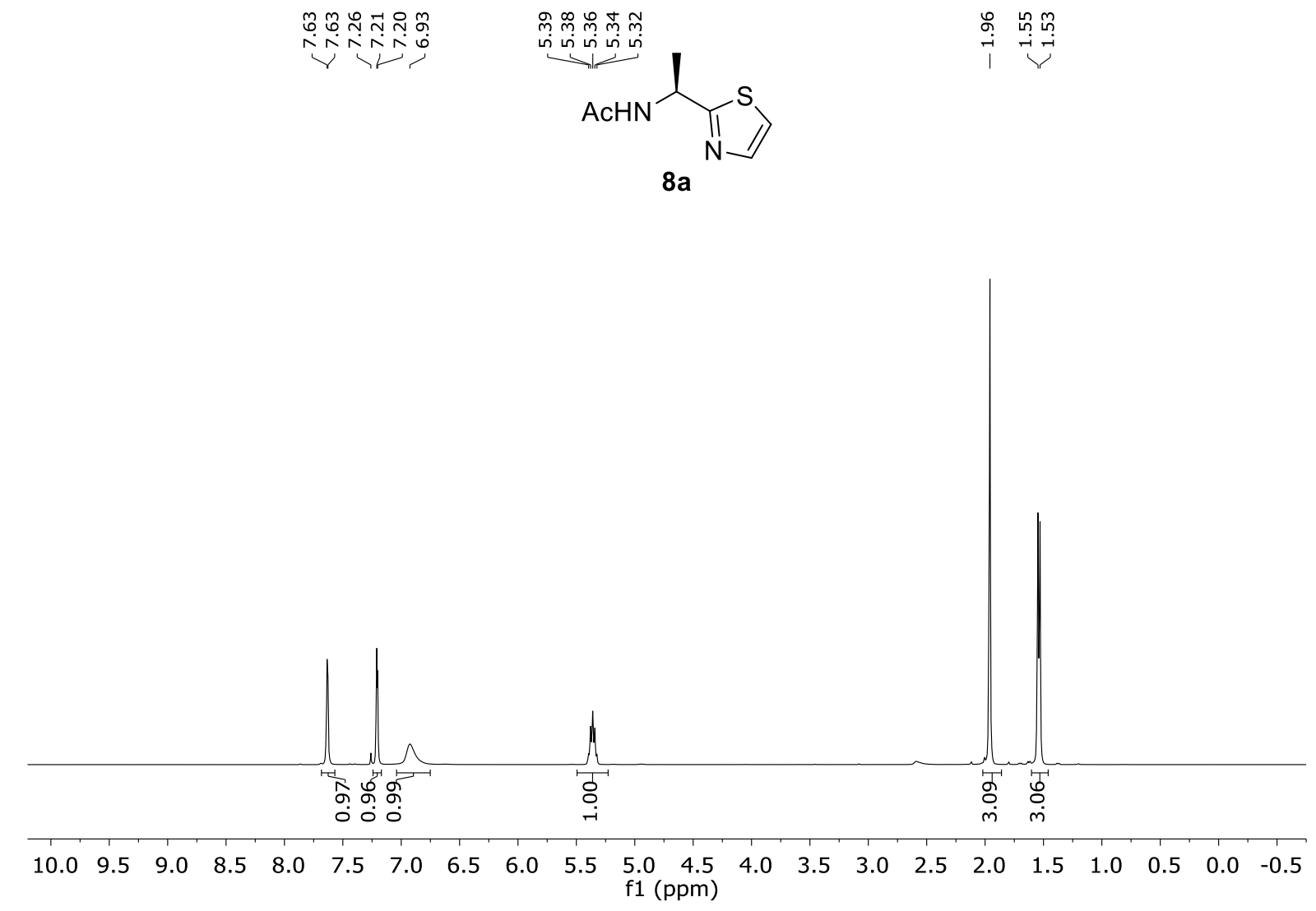

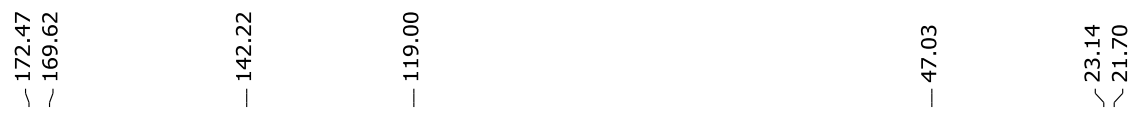

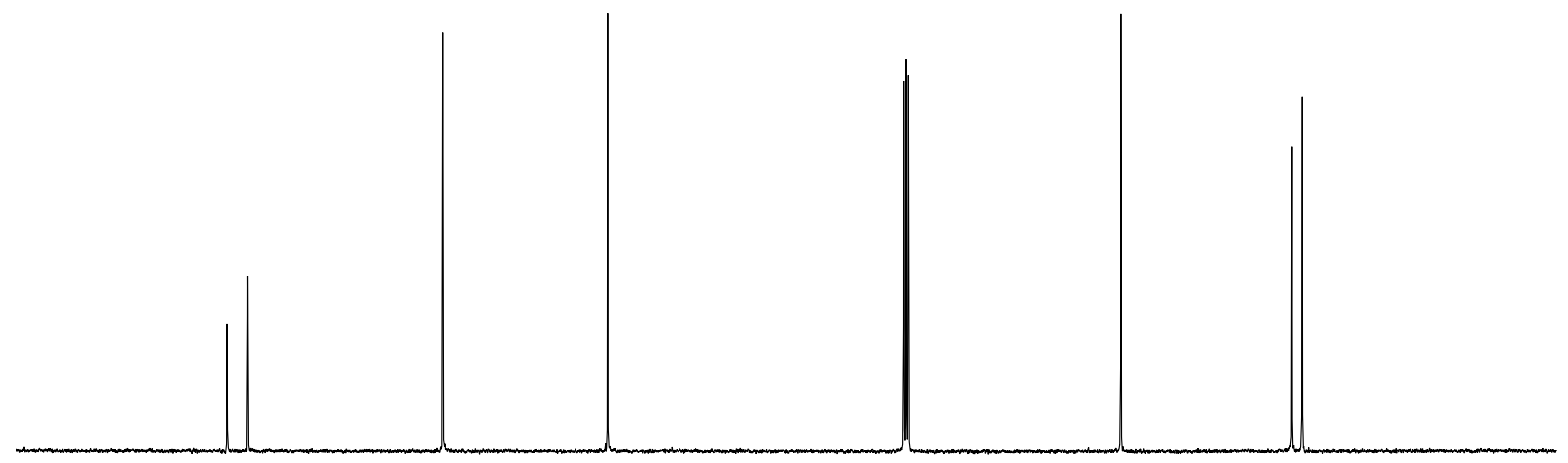

$\begin{array}{llllllllllllllllllllll}200 & 190 & 180 & 170 & 160 & 150 & 140 & 130 & 120 & 110 & \begin{array}{c}100 \\ \mathrm{f} 1(\mathrm{ppm})\end{array} & 80 & 70 & 60 & 50 & 40 & 30 & 20 & 10 & 0 & -10\end{array}$ 

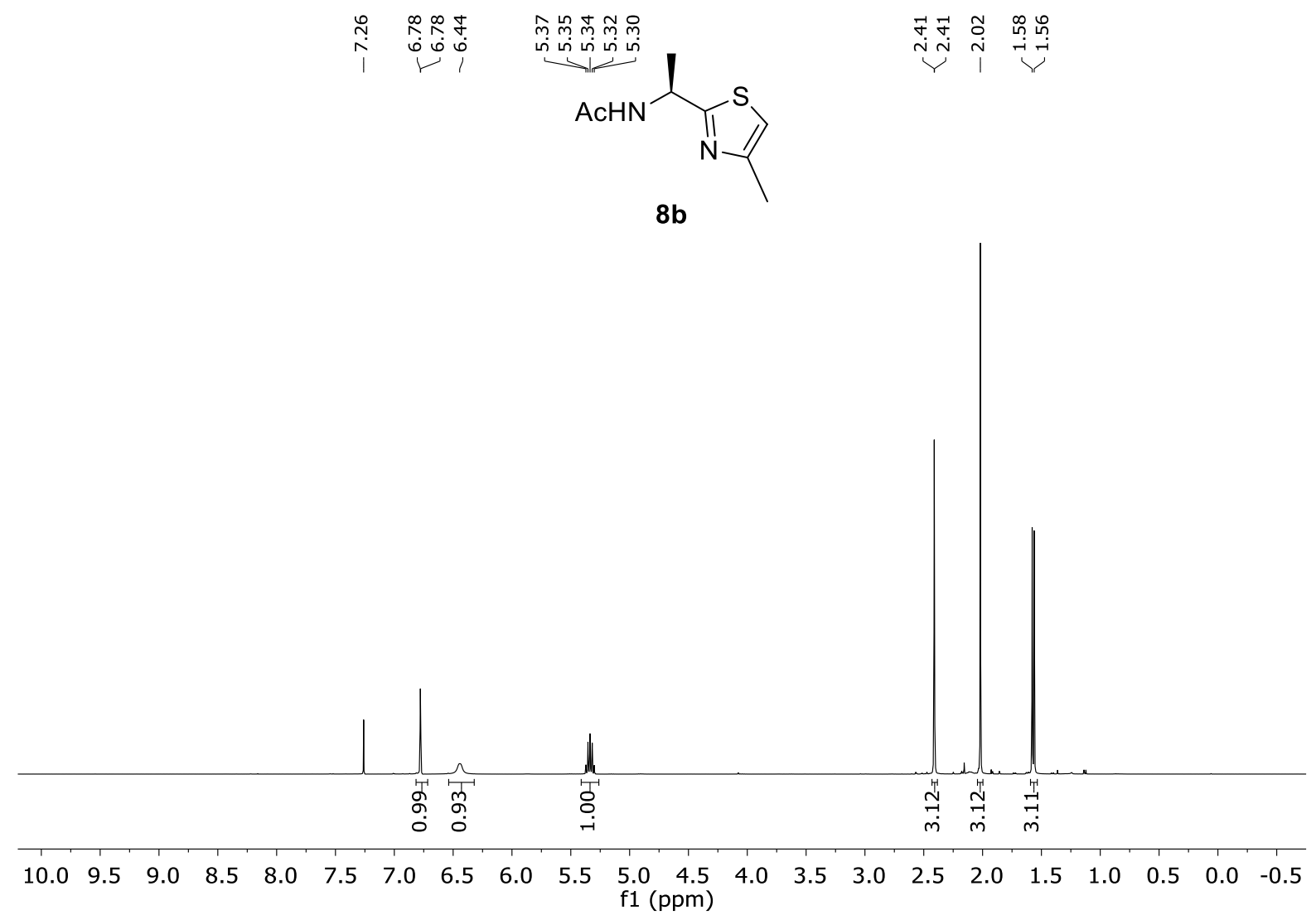

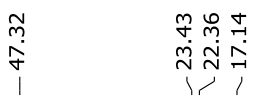

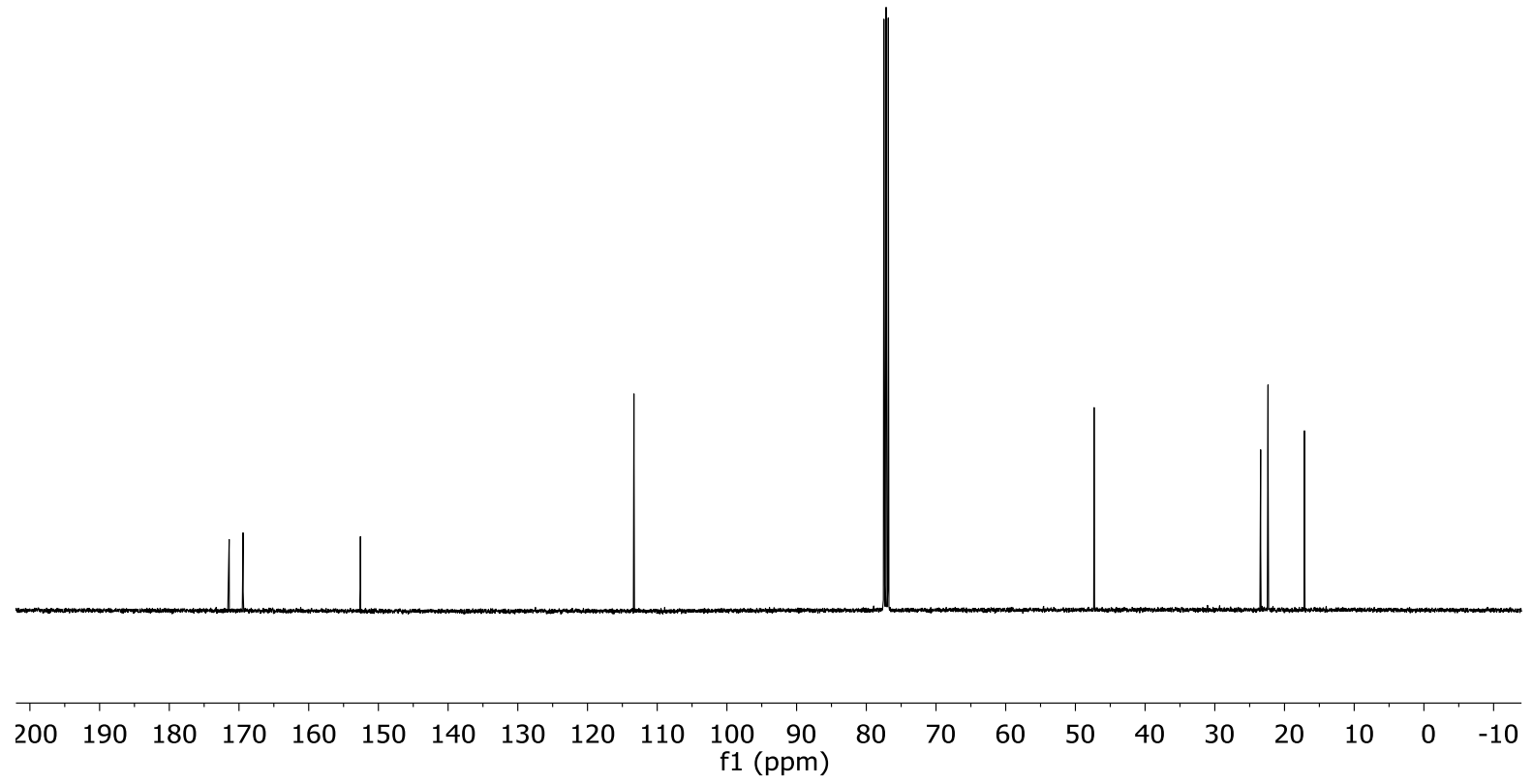




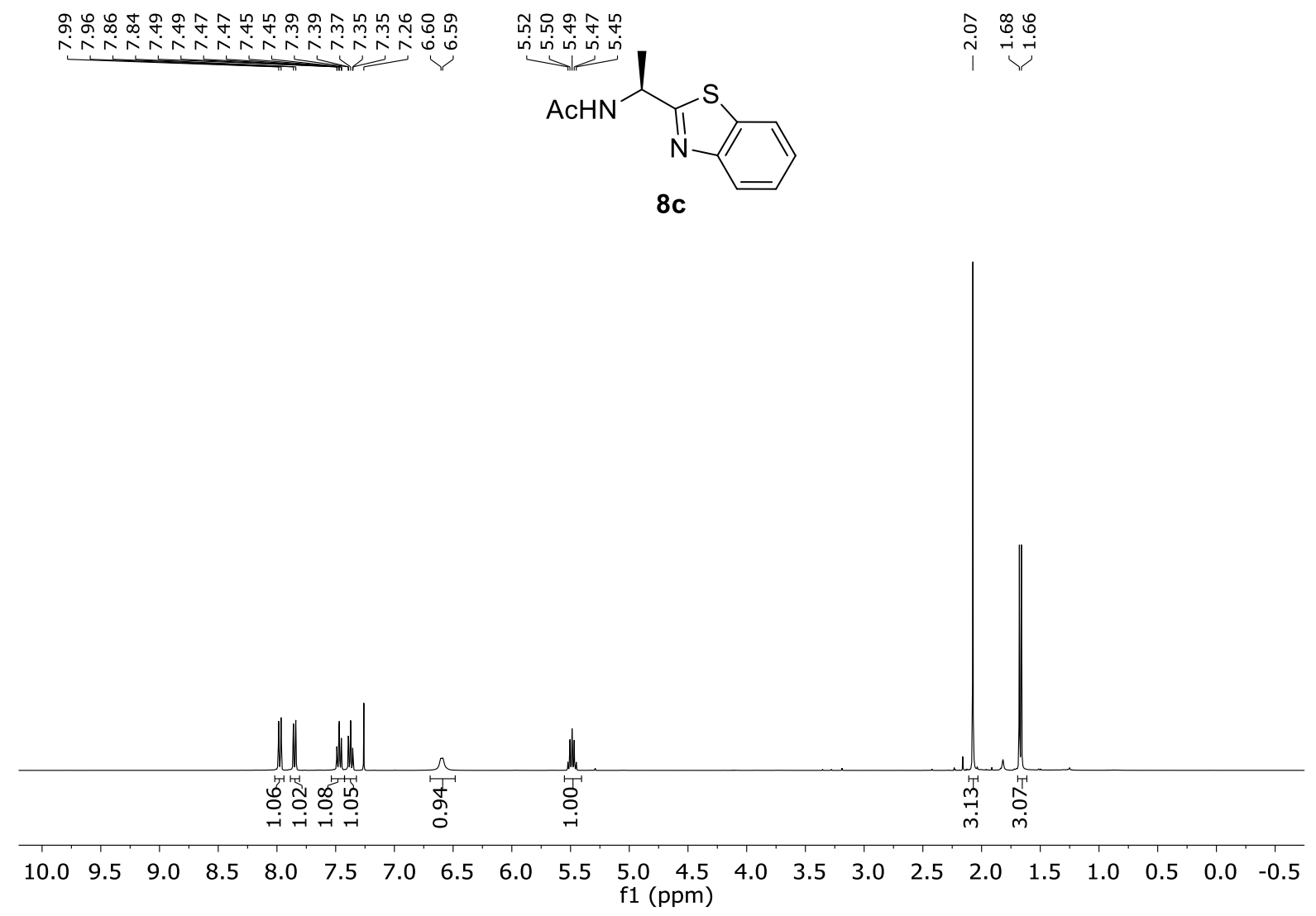

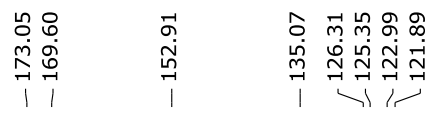

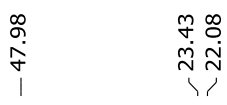

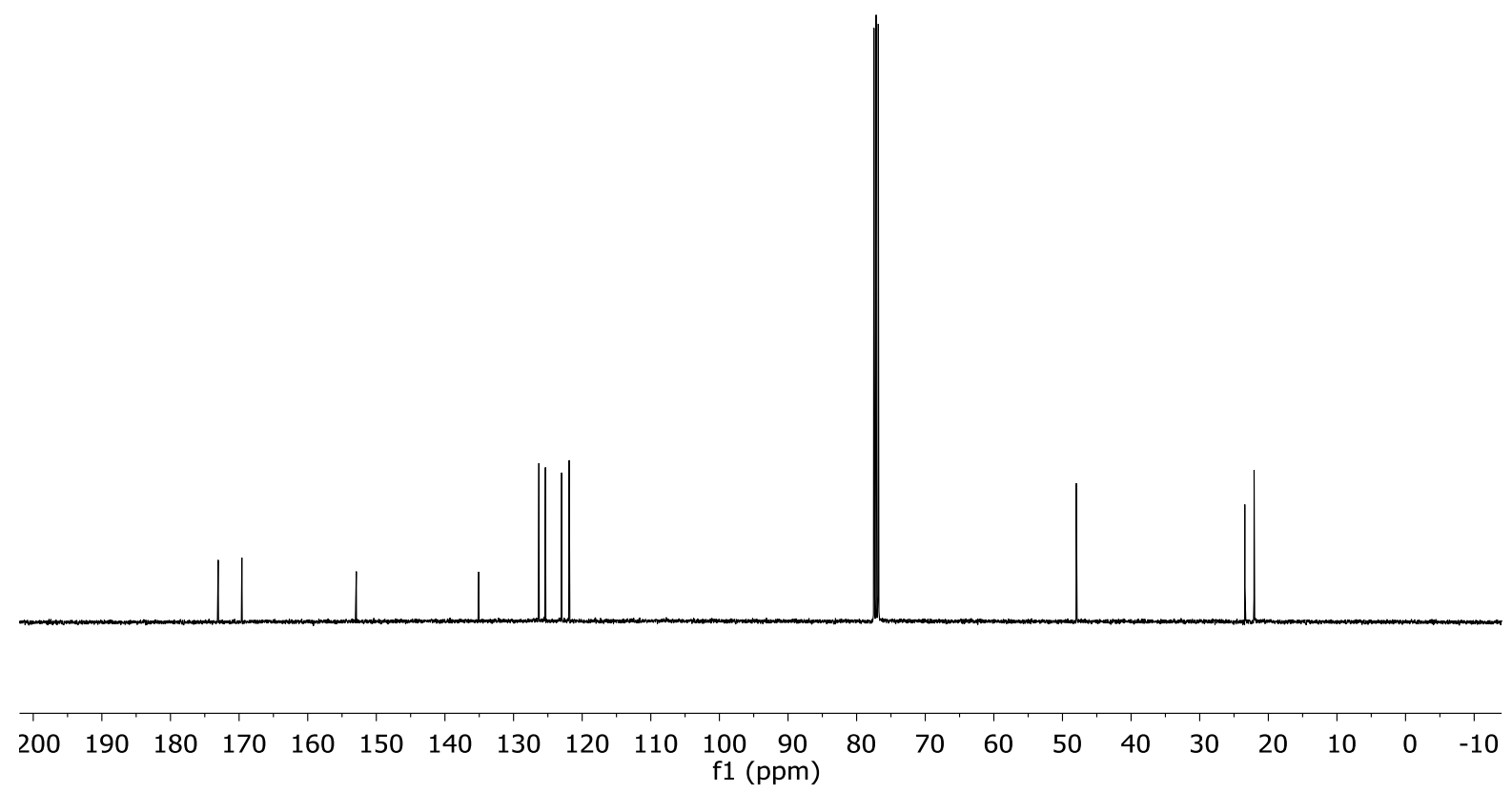




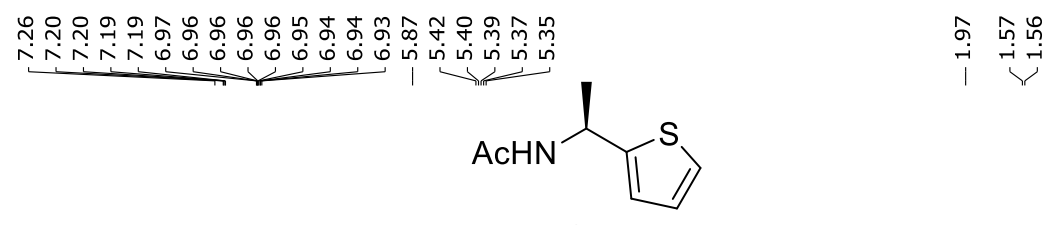

8d

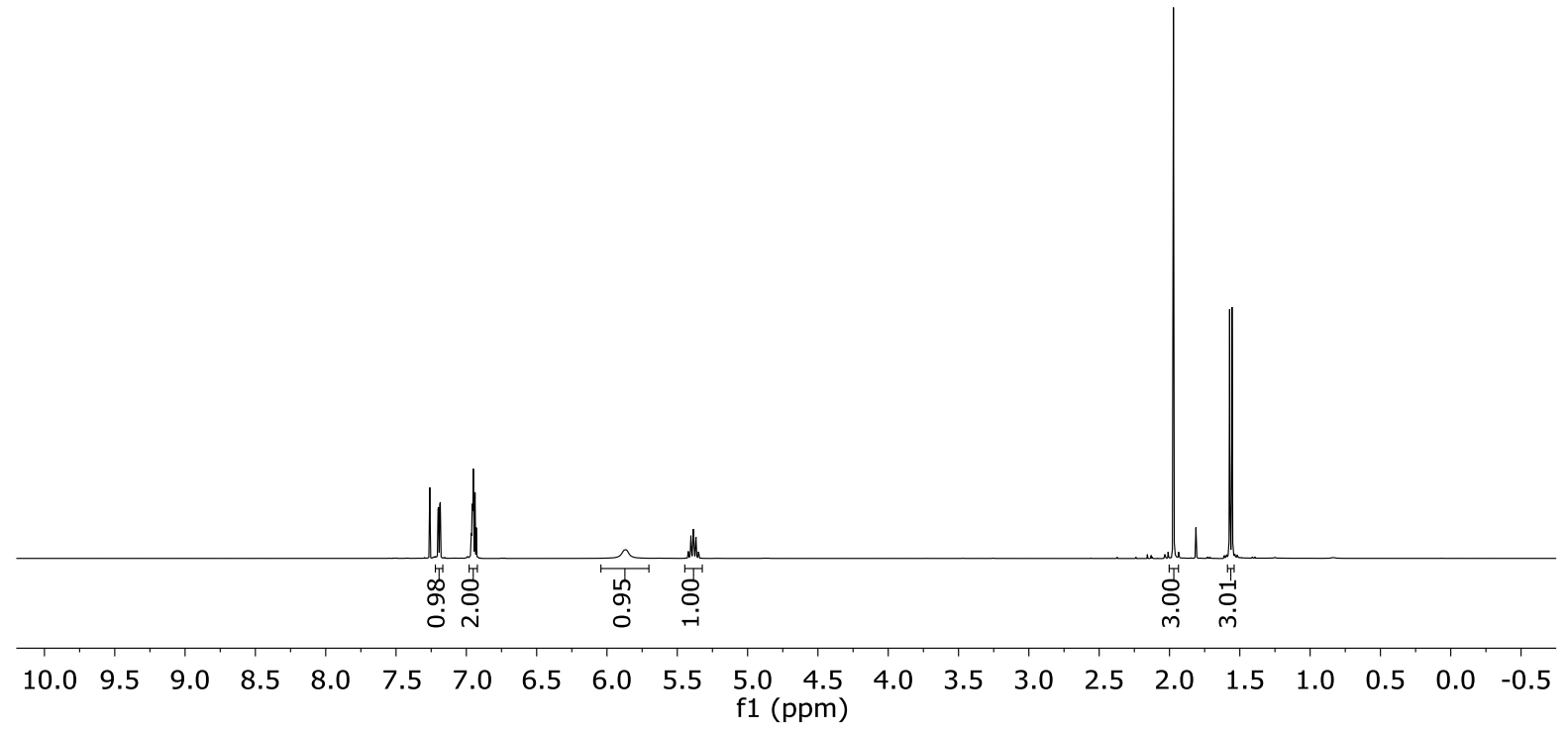

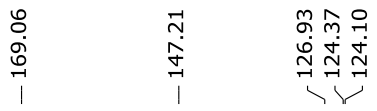

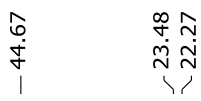

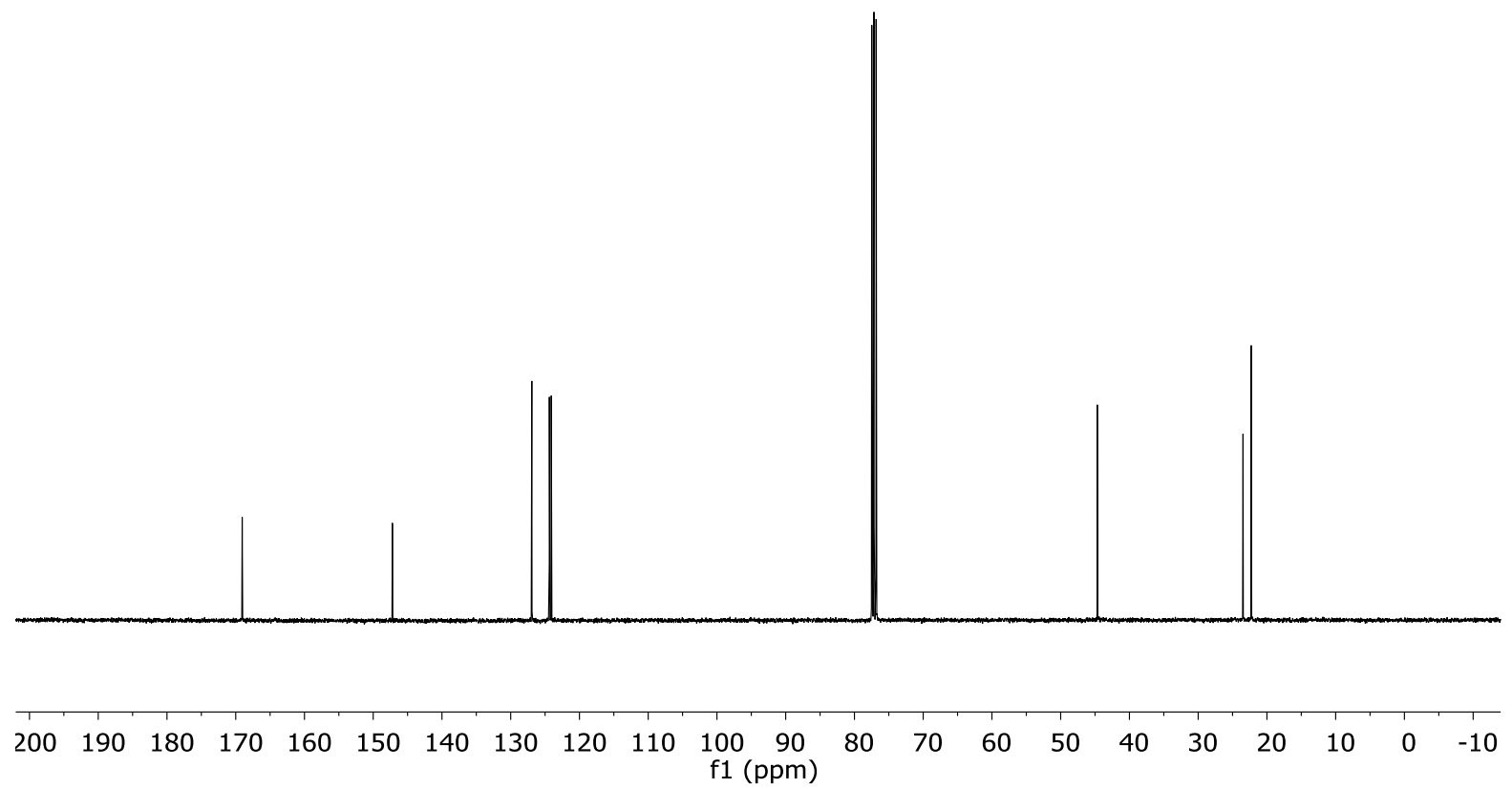




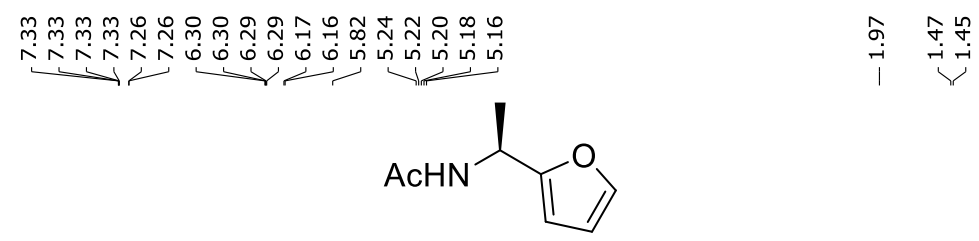

8 e
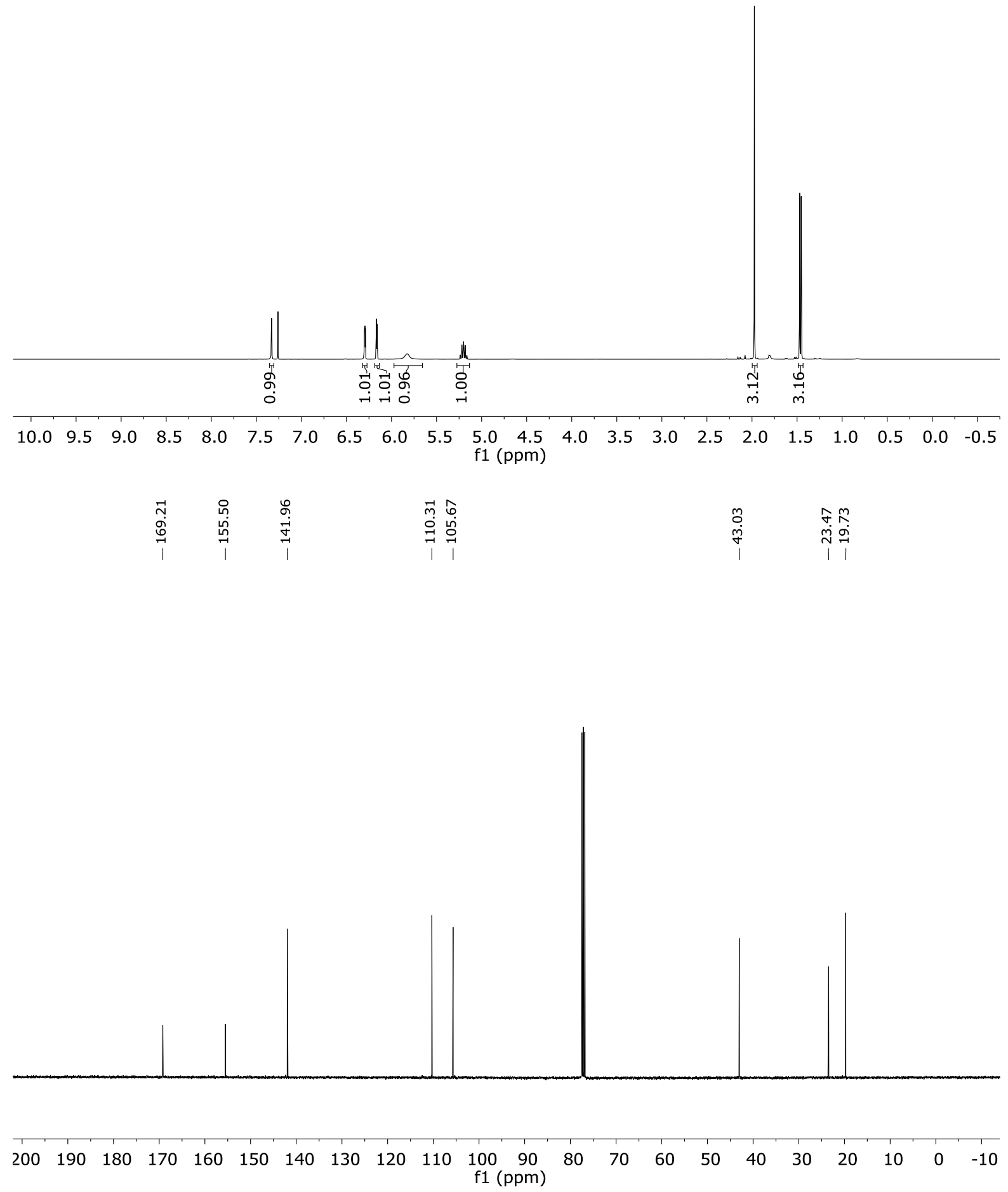

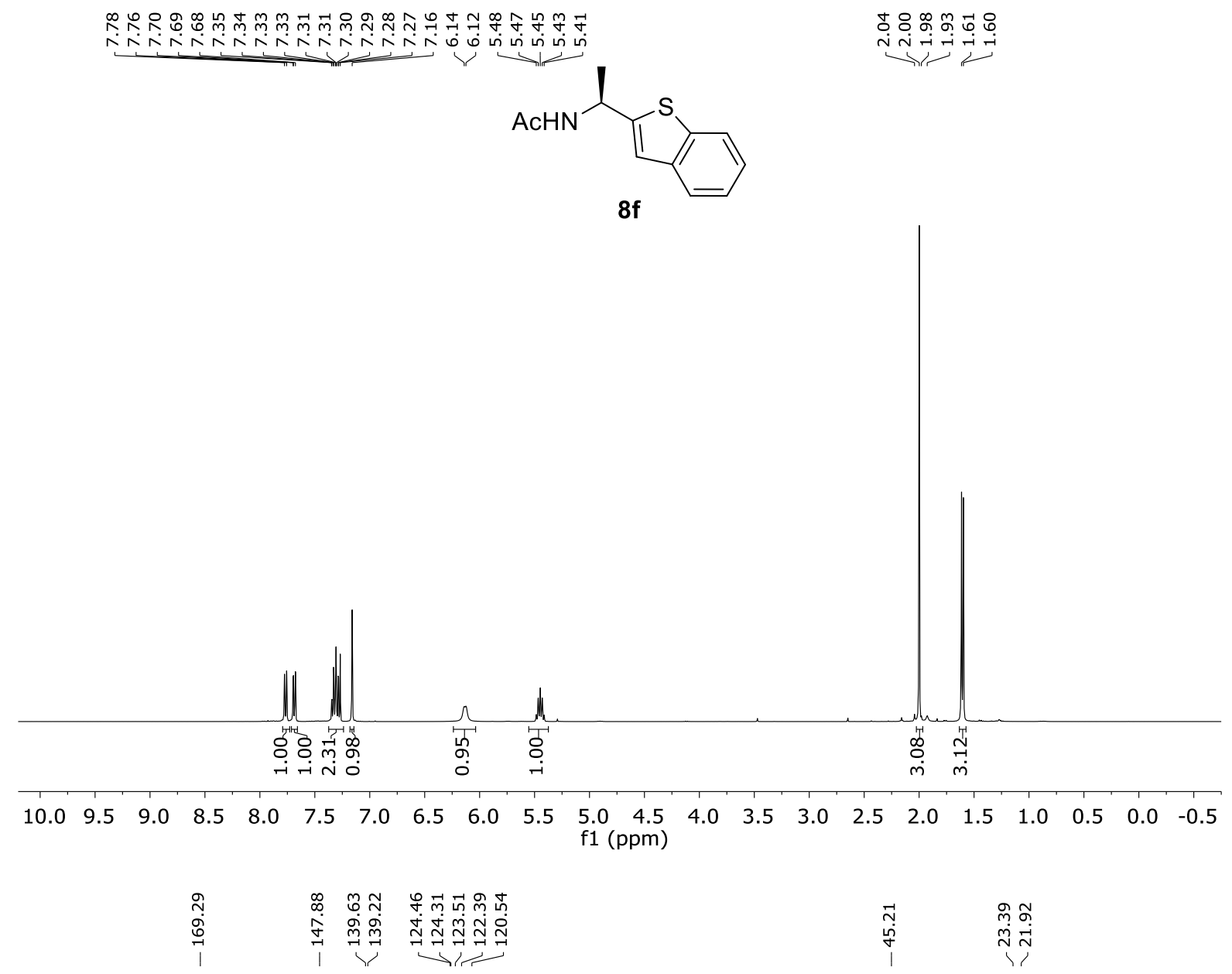

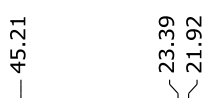

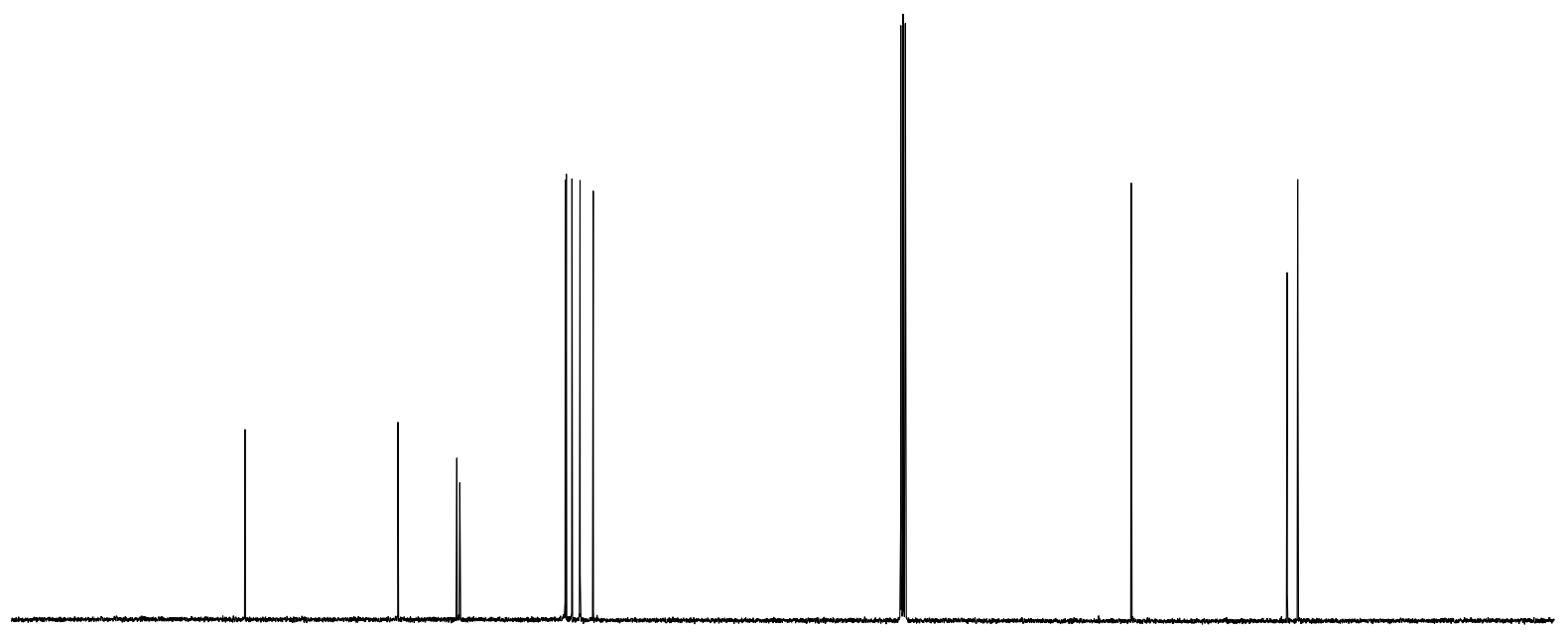

$\begin{array}{llllllllllllllllllllll}200 & 190 & 180 & 170 & 160 & 150 & 140 & 130 & 120 & 110 & \underset{f 100}{\mathrm{f} 1(\mathrm{ppm})} & 90 & 80 & 70 & 60 & 50 & 40 & 30 & 20 & 10 & 0 & -10\end{array}$ 


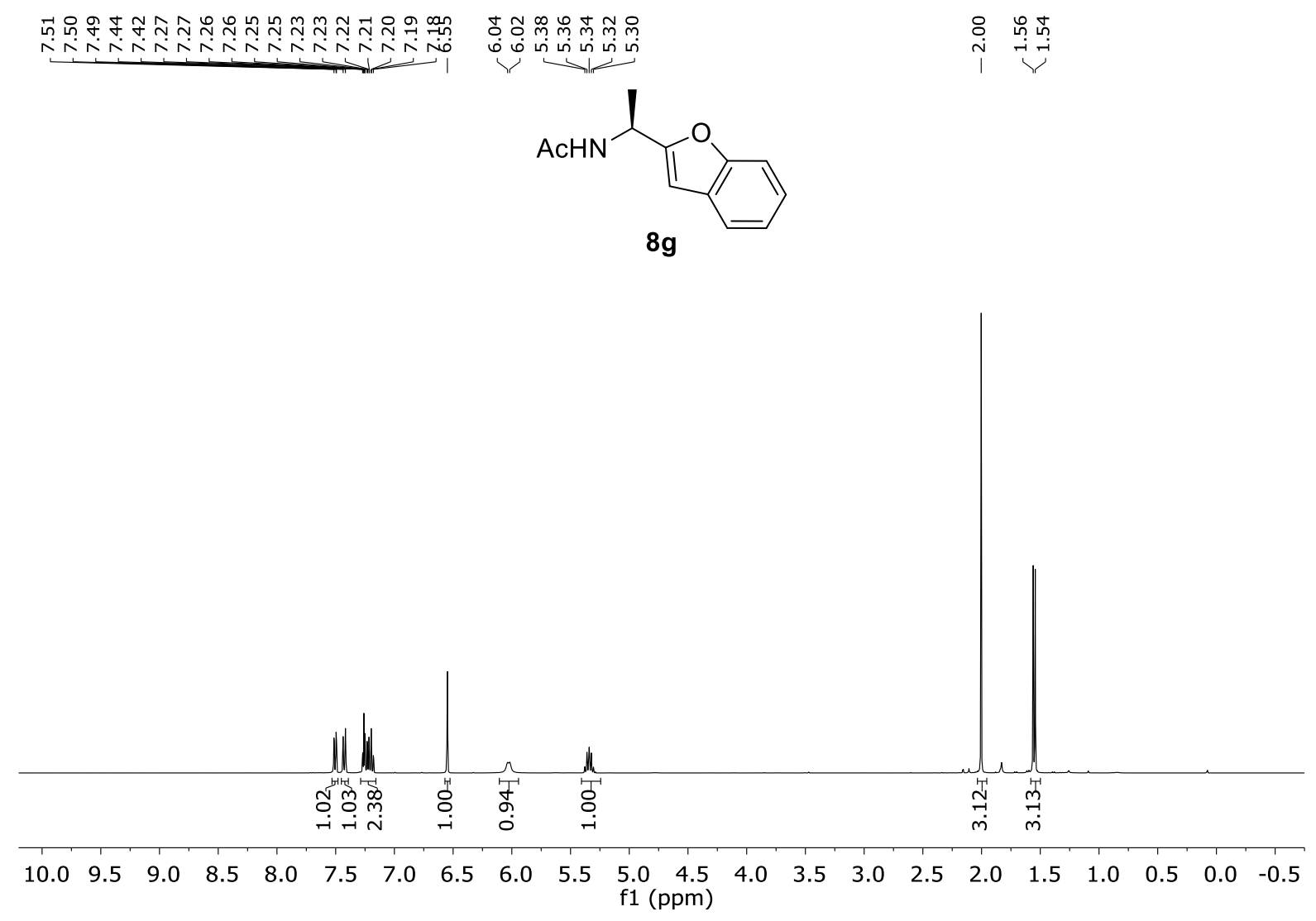

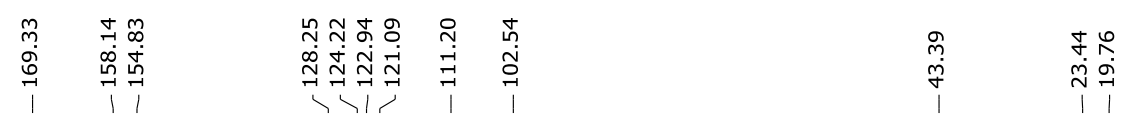

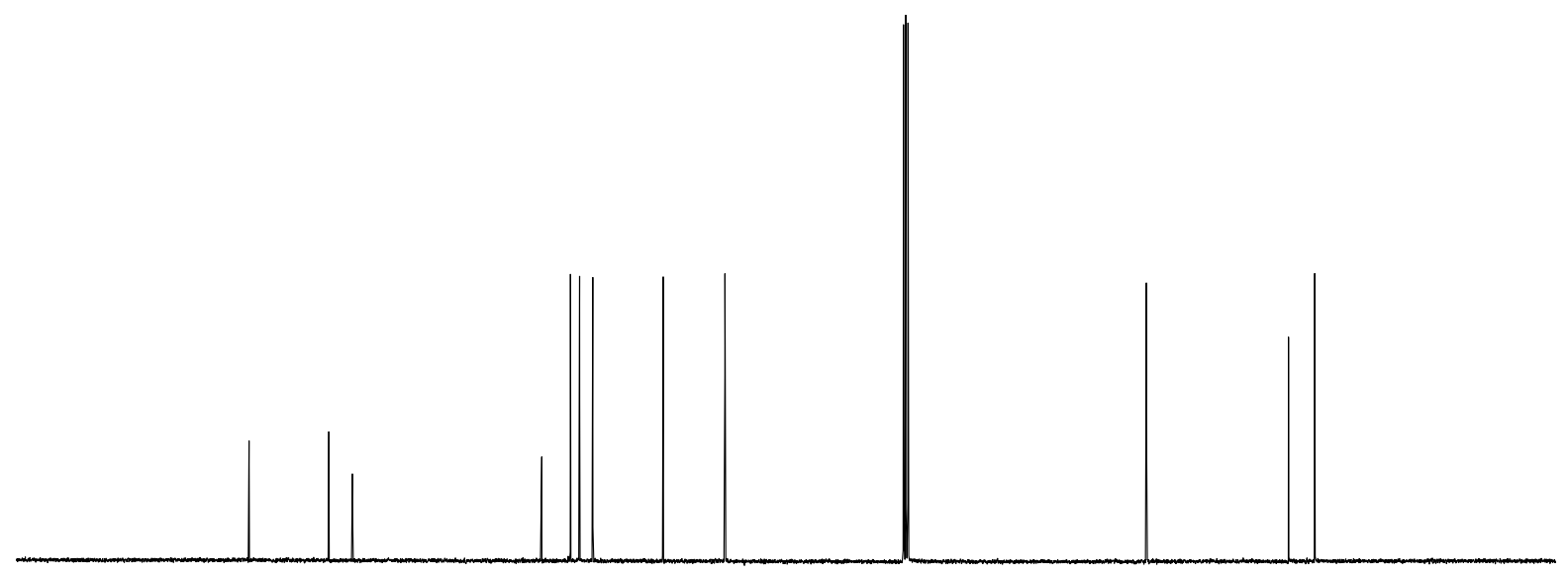

$\begin{array}{llllllllllllllllllllll}200 & 190 & 180 & 170 & 160 & 150 & 140 & 130 & 120 & 110 & \underset{f 1}{\mathrm{f} 1}(\mathrm{ppm}) & 90 & 80 & 70 & 60 & 50 & 40 & 30 & 20 & 10 & 0 & -10\end{array}$ 

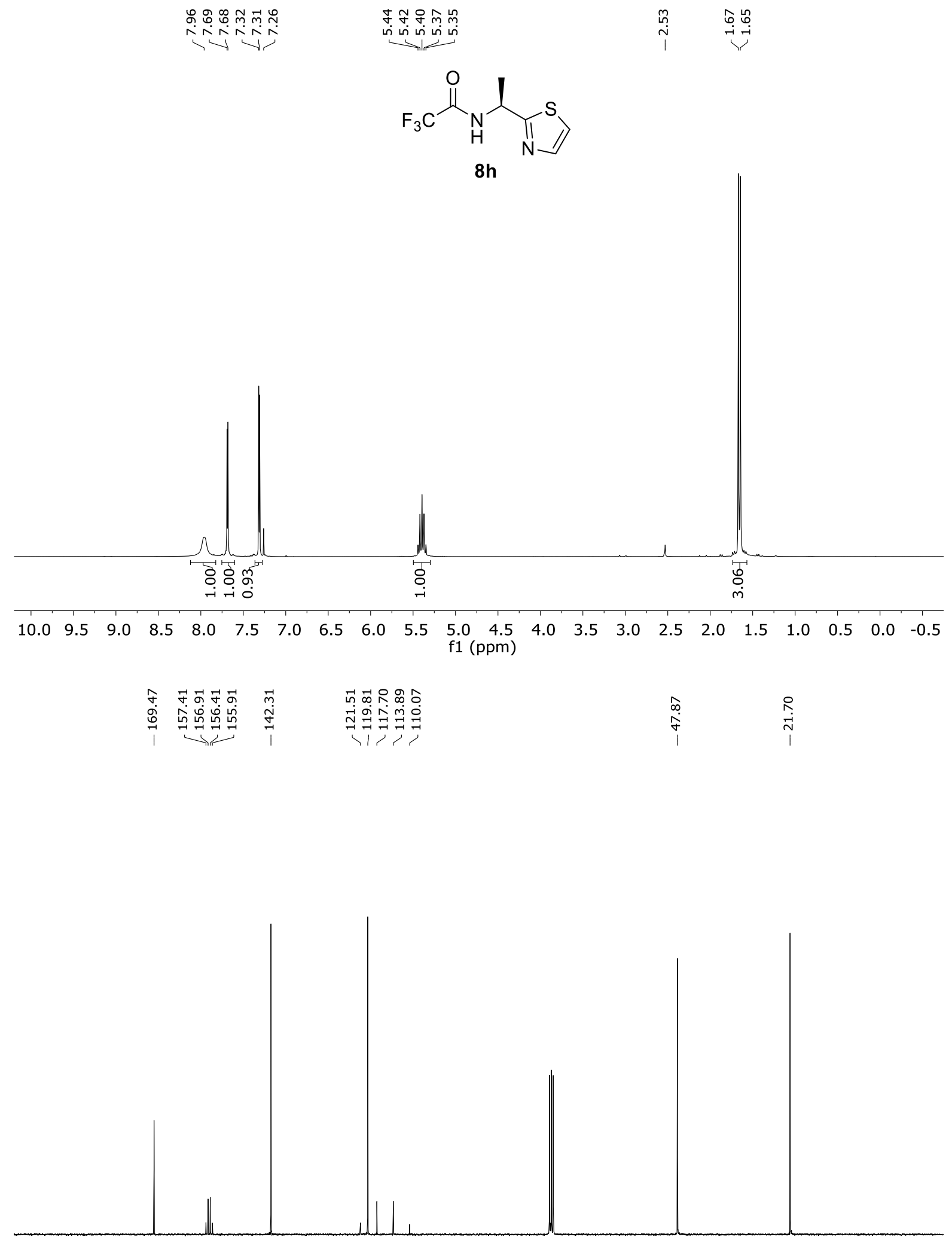

$\begin{array}{llllllllllllllllllllll}200 & 190 & 180 & 170 & 160 & 150 & 140 & 130 & 120 & 110 & \underset{f 100}{\mathrm{f} 1(\mathrm{ppm})} & 90 & 80 & 70 & 60 & 50 & 40 & 30 & 20 & 10 & 0 & -10\end{array}$ 


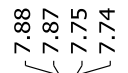

in

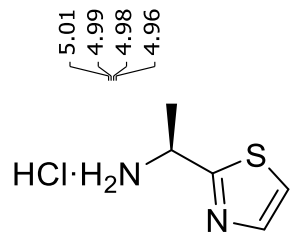

9. $\mathrm{HCl}$
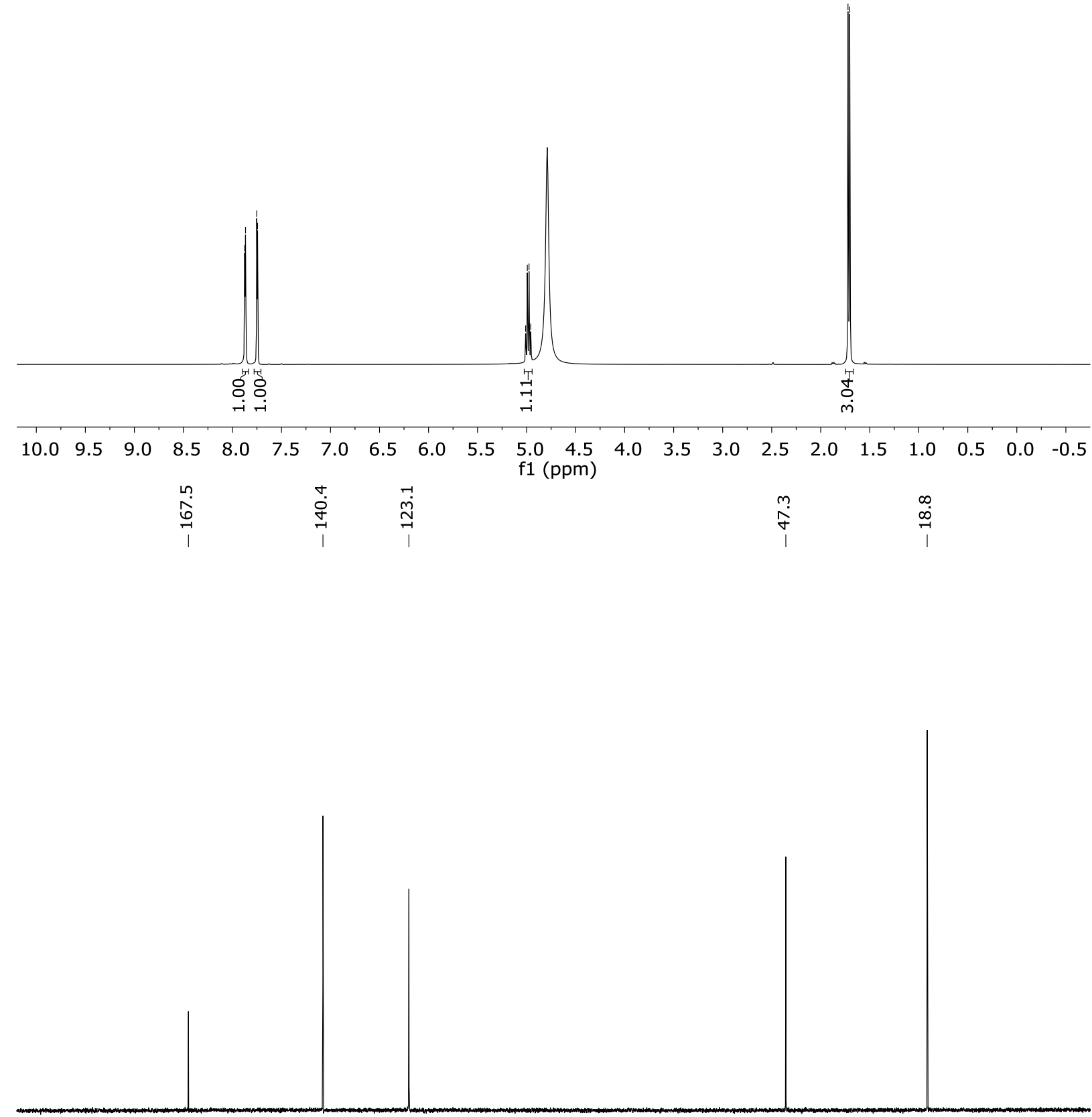

$\begin{array}{llllllllllllllllllllll}200 & 190 & 180 & 170 & 160 & 150 & 140 & 130 & 120 & 110 & \begin{array}{c}100 \\ \mathrm{f} 1(\mathrm{ppm})\end{array} & 80 & 70 & 60 & 50 & 40 & 30 & 20 & 10 & 0 & -10\end{array}$ 


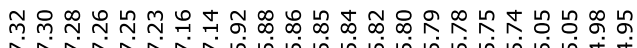

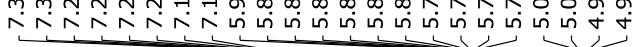

i<smiles>C=CCCC/C(=C\I)Cc1ccccc1</smiles>

$11 \mathrm{i}$
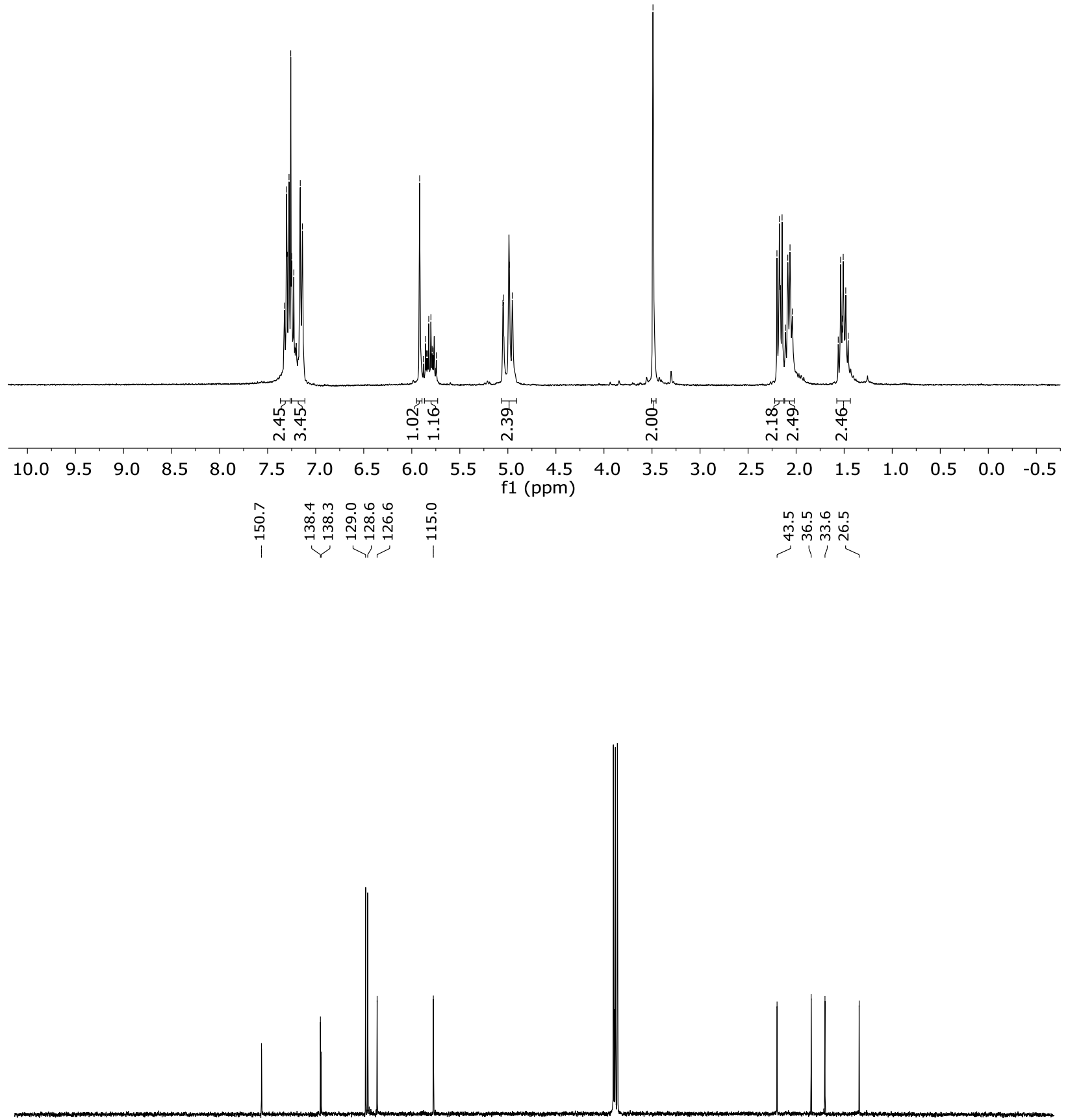

$\begin{array}{llllllllllllllllllllll}200 & 190 & 180 & 170 & 160 & 150 & 140 & 130 & 120 & 110 & 100 & 90 & 80 & 70 & 60 & 50 & 40 & 30 & 20 & 10 & 0 & -10\end{array}$

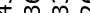



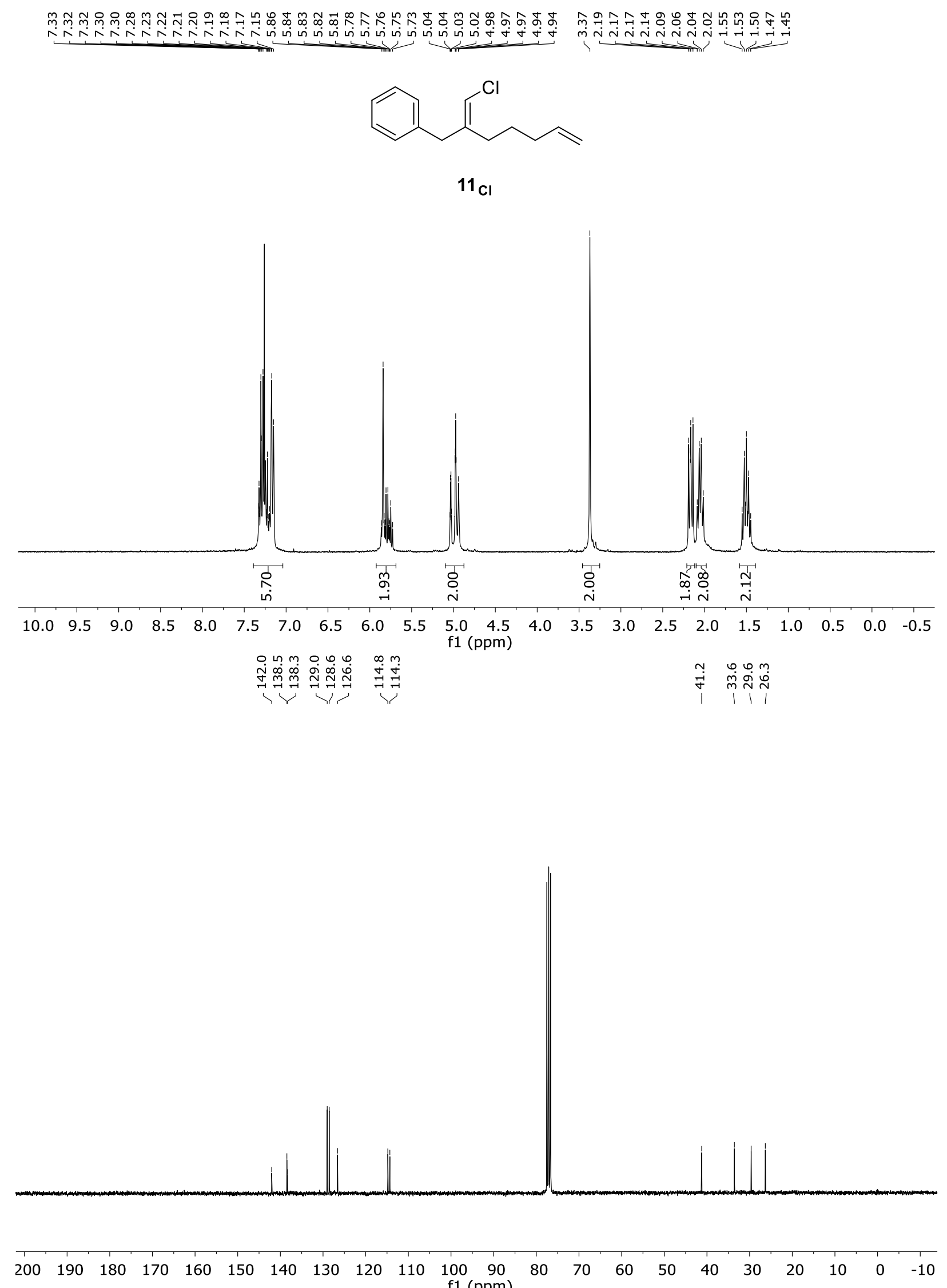
م.

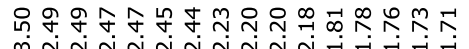

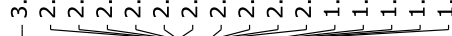<smiles>O=CCCC/C(=C\I)Cc1ccccc1</smiles>
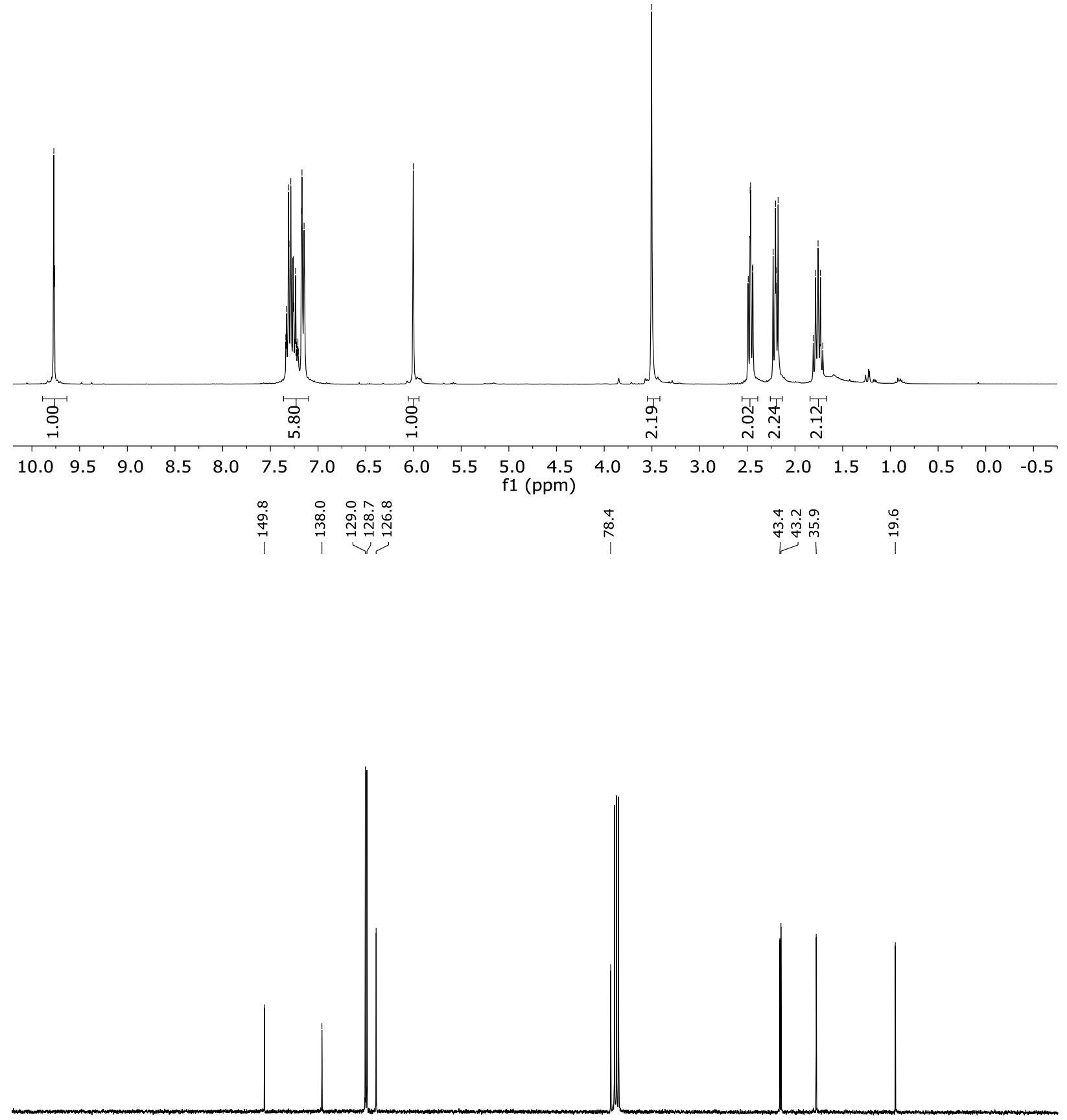

$\begin{array}{llllllllllllllllllllll}200 & 190 & 180 & 170 & 160 & 150 & 140 & 130 & 120 & 110 & 100 & 90 & 80 & 70 & 60 & 50 & 40 & 30 & 20 & 10 & 0 & -10\end{array}$ 


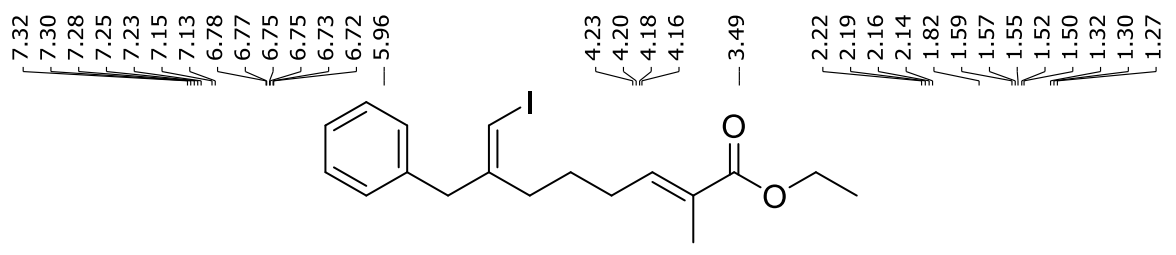

$12_{\mathrm{i}}$
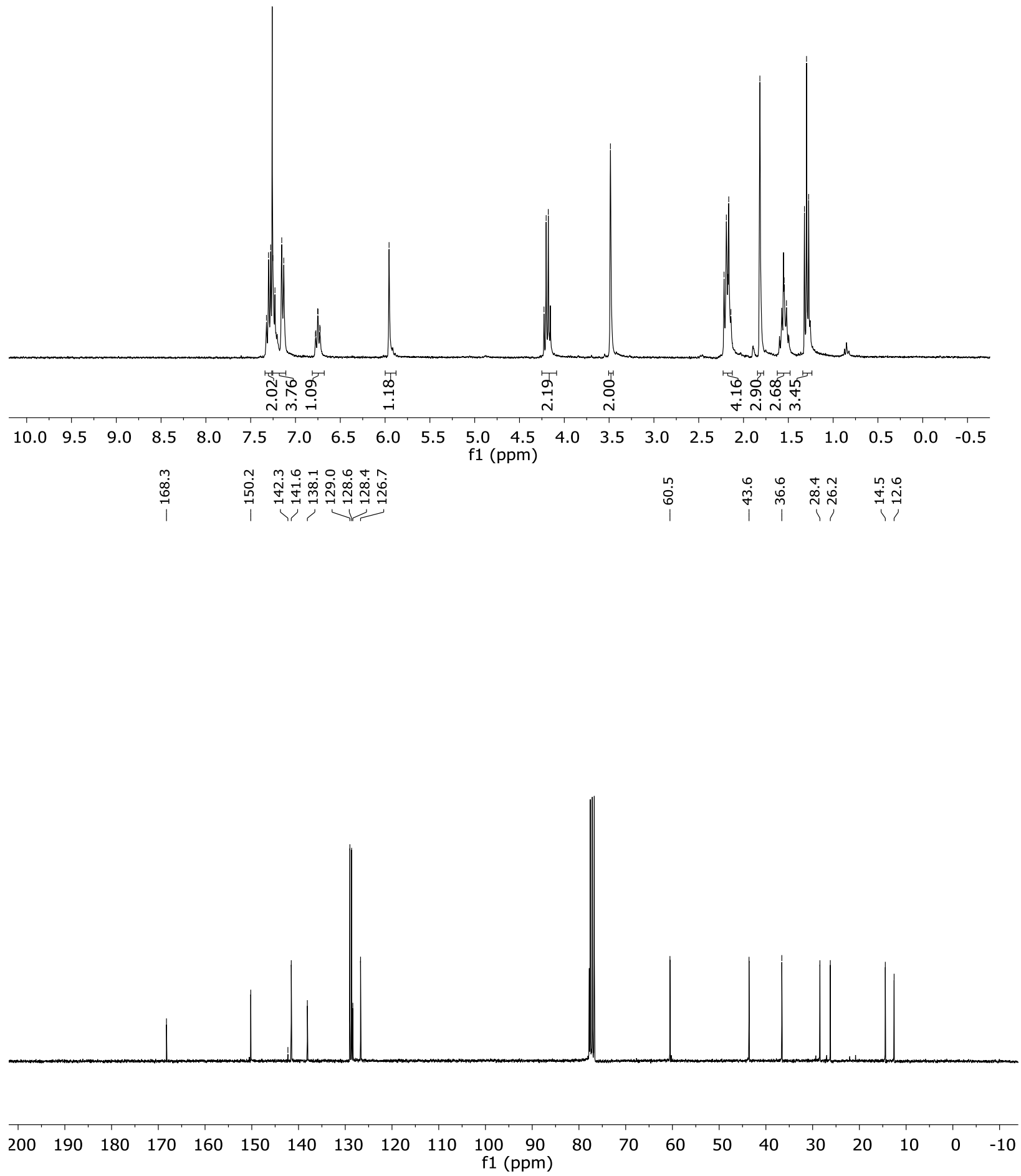


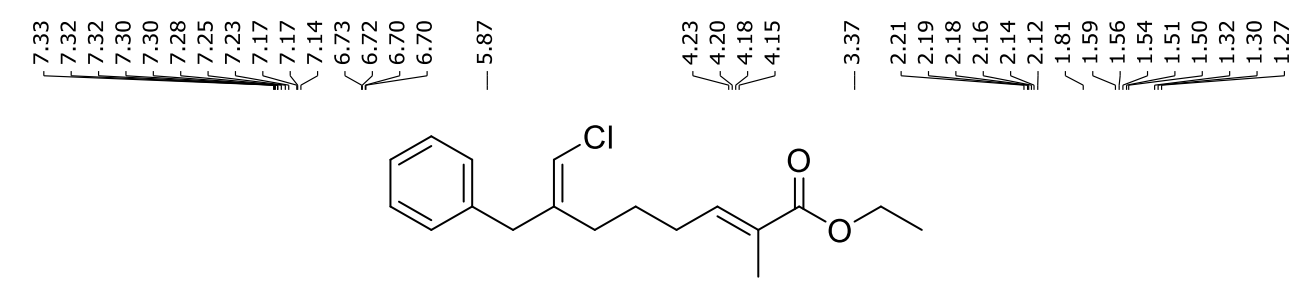

$12_{\mathrm{Cl}}$
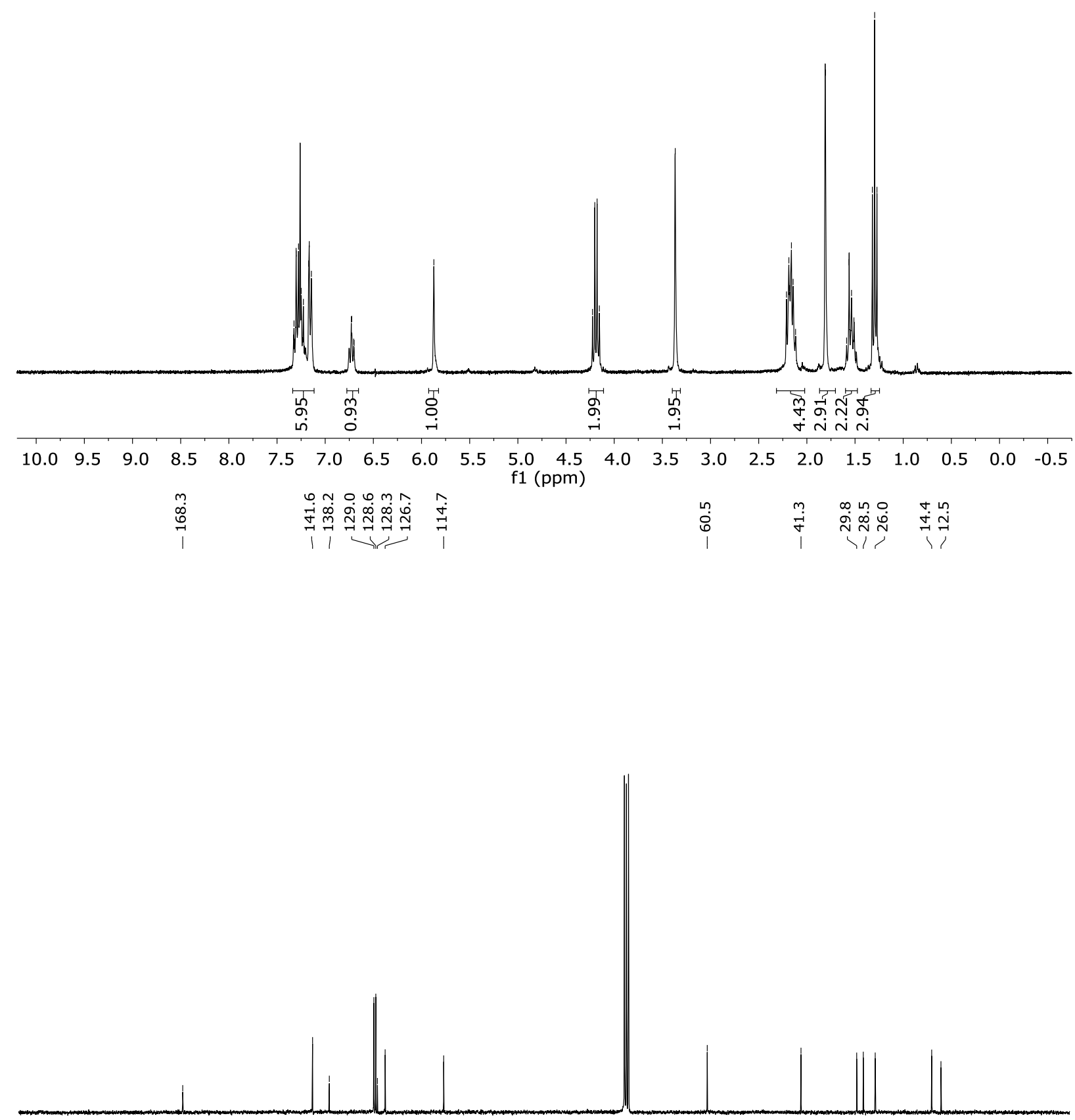


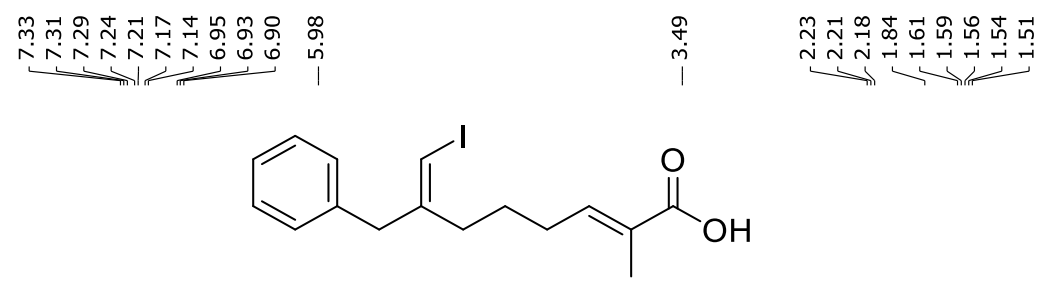

$13 i$
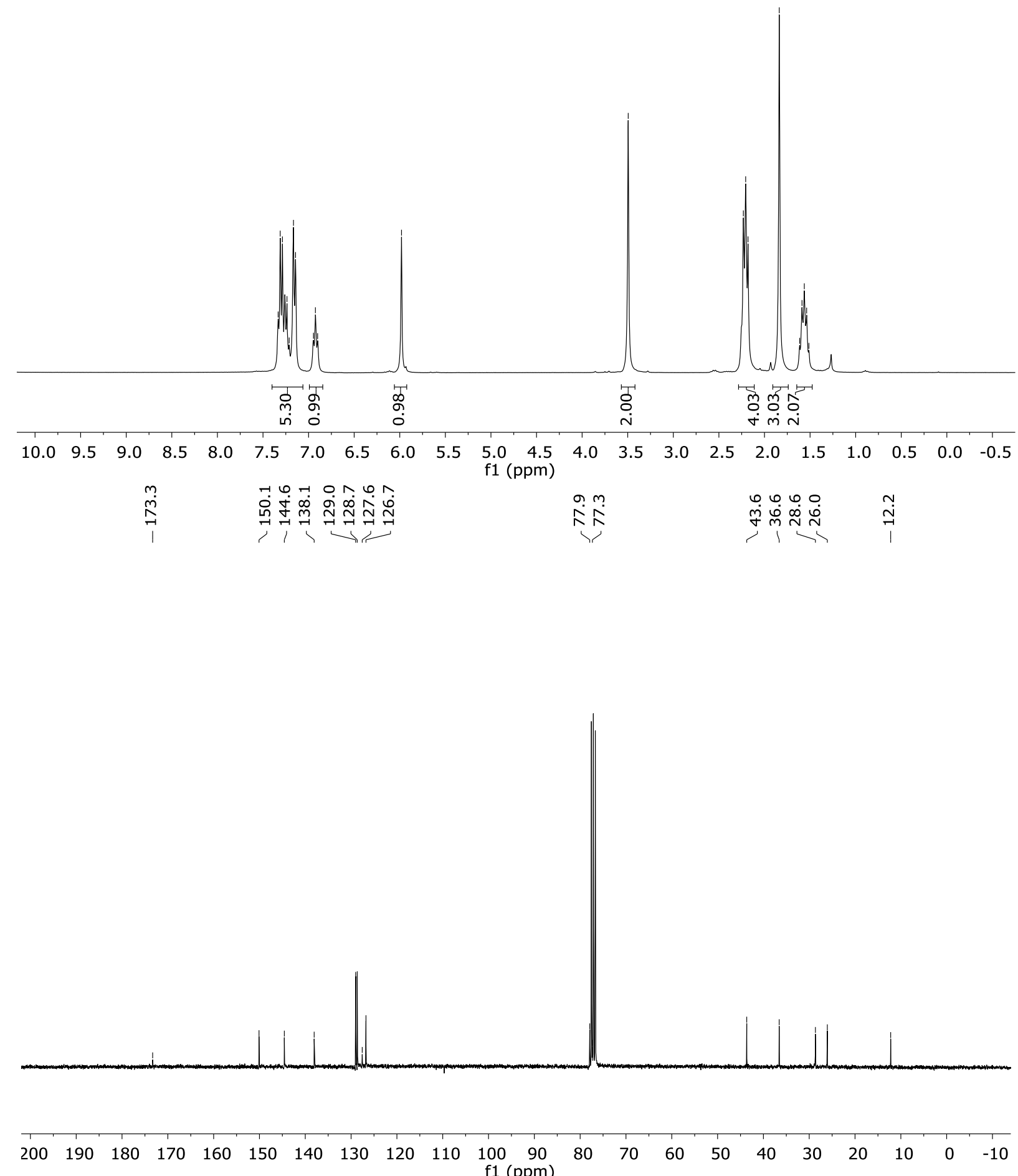

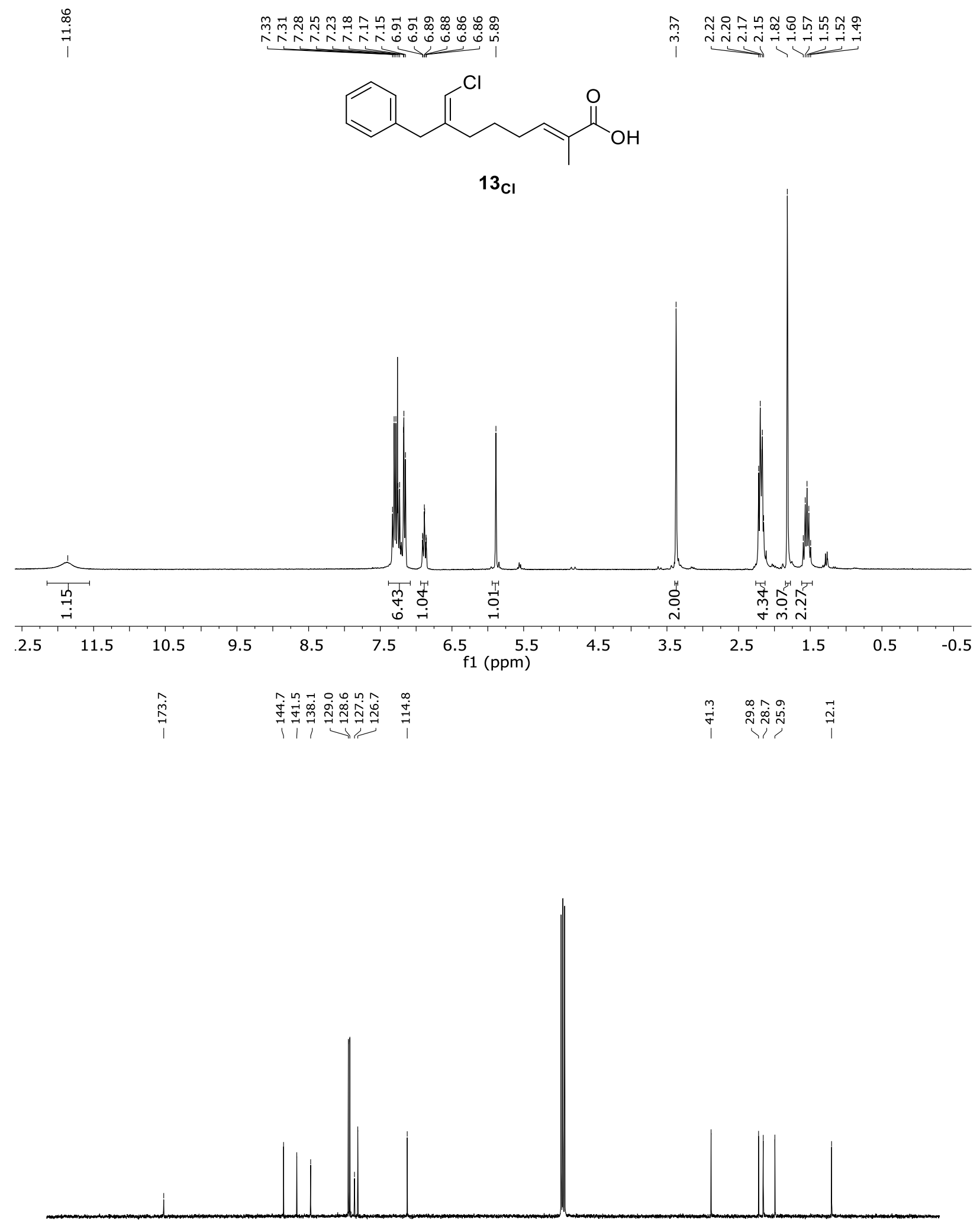

$\begin{array}{rlllllllllllllllllllll}200 & 190 & 180 & 170 & 160 & 150 & 140 & 130 & 120 & 110 & \underset{f 1}{100}(\mathrm{ppm}) & 80 & 70 & 60 & 50 & 40 & 30 & 20 & 10 & 0 & -10\end{array}$ 


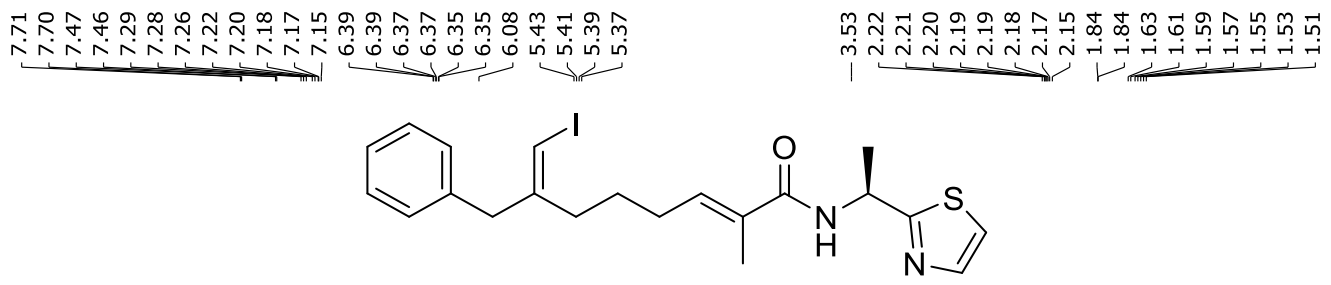

iodo-conulothiazole $\mathrm{A}\left(\mathbf{1}_{\mathbf{i}}\right)$
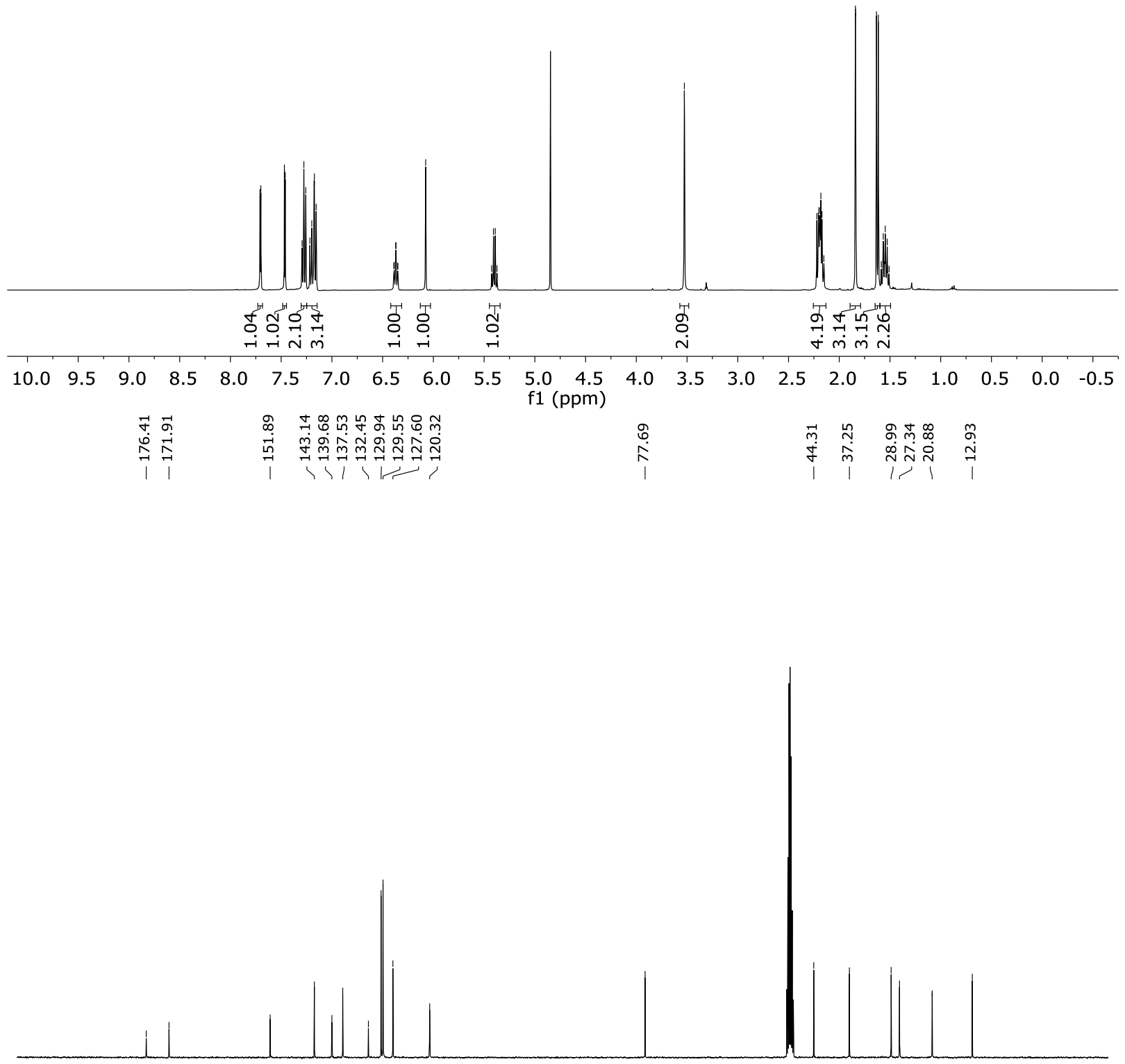

$\begin{array}{llllllllllllllllllllllllll}200 & 190 & 180 & 170 & 160 & 150 & 140 & 130 & 120 & 110 & \begin{array}{c}100 \\ \mathrm{f} 1(\mathrm{ppm})\end{array} & \begin{array}{c}90 \\ (\mathrm{pm})\end{array} & 70 & 60 & 50 & 40 & 30 & 20 & 10 & 0 & -10\end{array}$




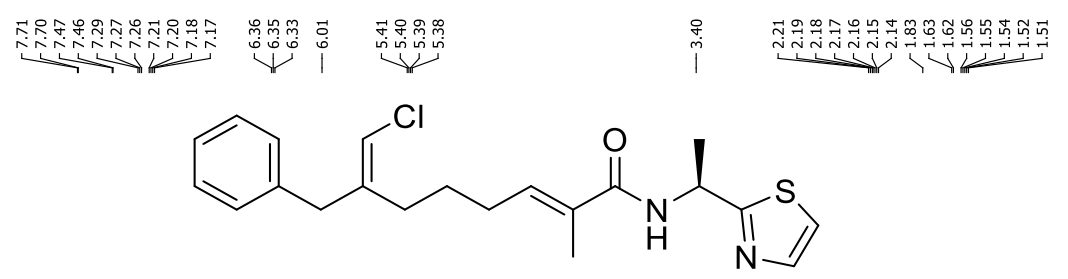

conulothiazole A (1)
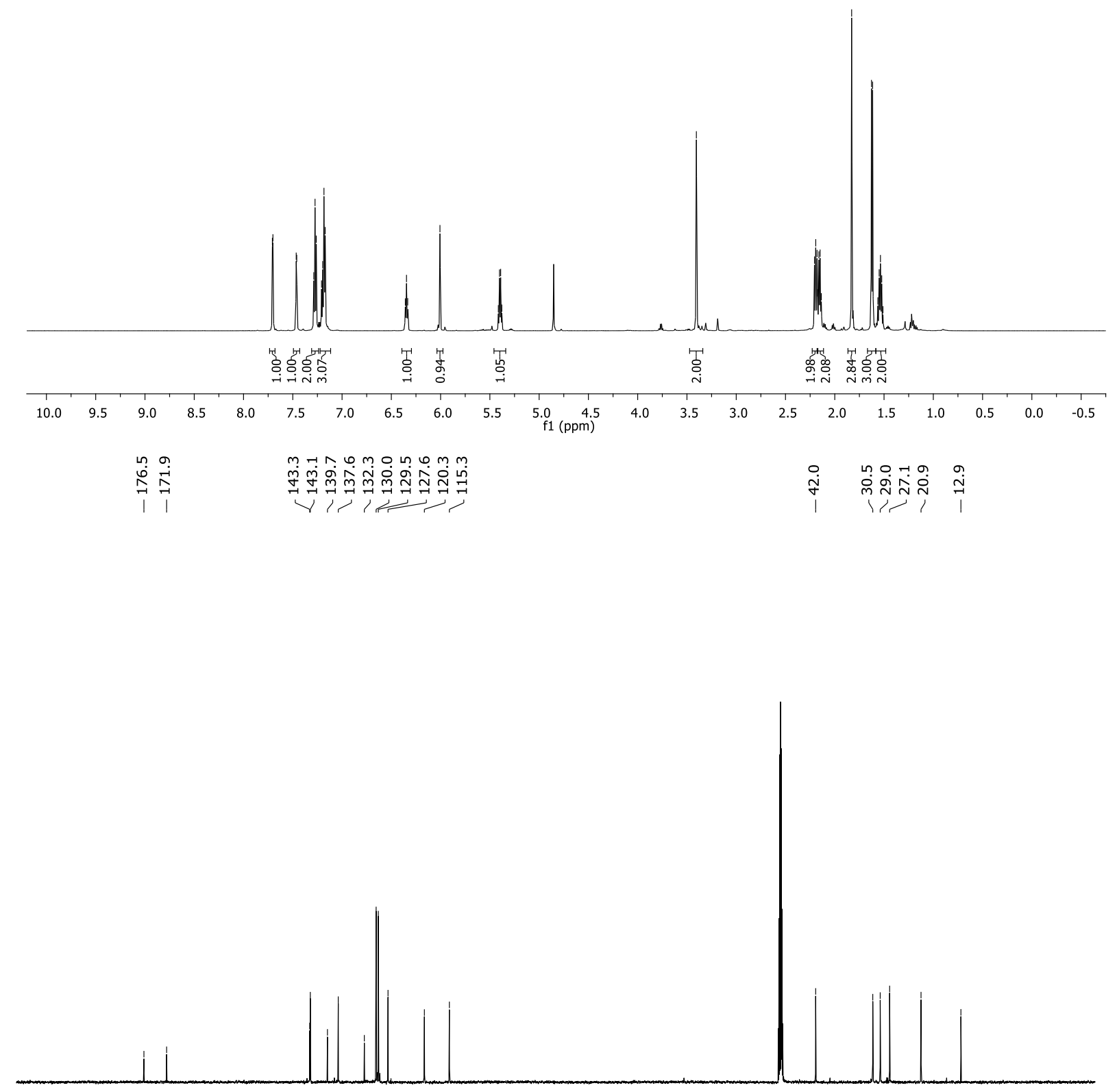

$\begin{array}{llllllllllllllllllllllll}200 & 190 & 180 & 170 & 160 & 150 & 140 & 130 & 120 & 110 & 100 & 90 & 80 & 70 & 60 & 50 & 40 & 30 & 20 & 10 & 0 & -10\end{array}$ 